Sediment Pore-Water Toxicity Test Results and Preliminary Toxicity Identification of Post-Landfall Pore-Water Samples Collected Following the Deepwater Horizon Oil Release, Gulf of Mexico, 2010
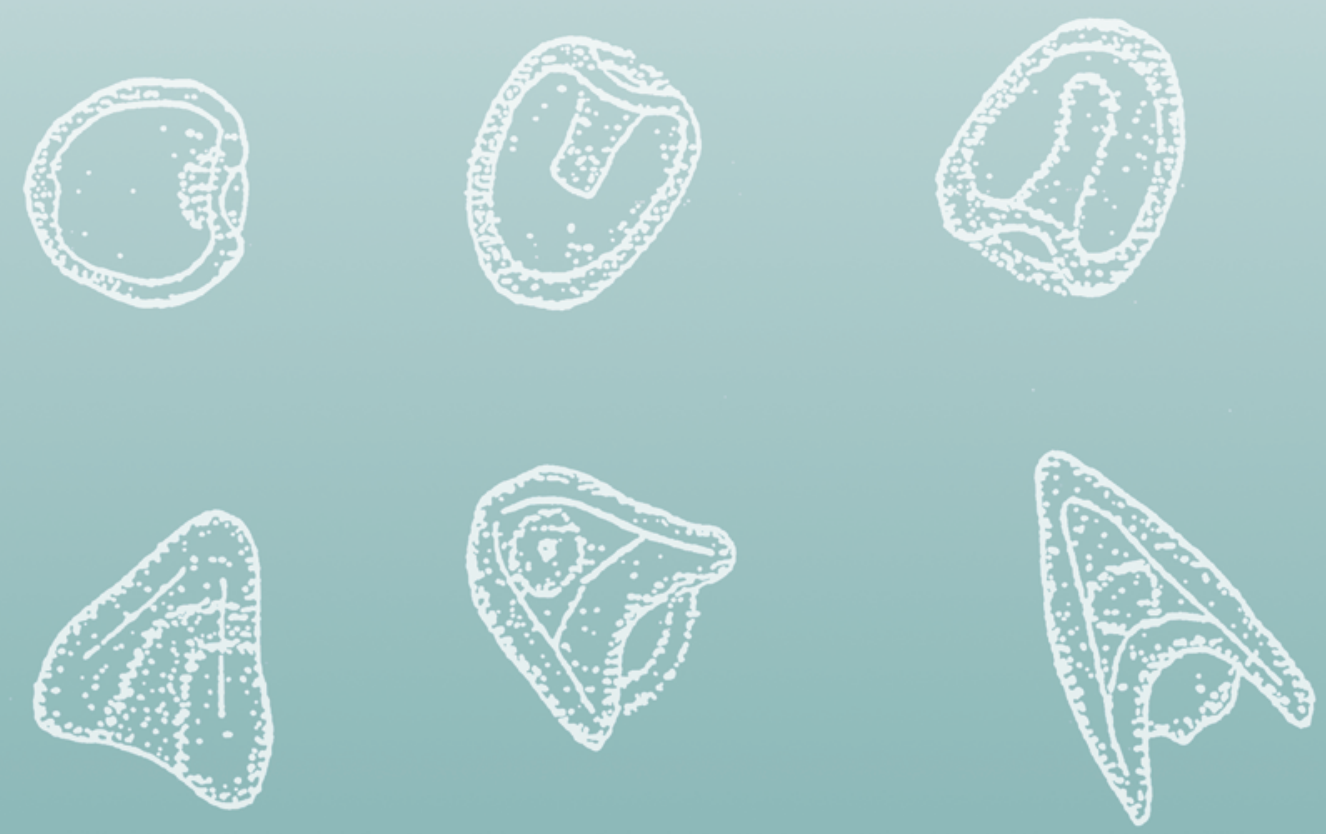

Open-File Report 2011-1078 
Cover. Artist's sketches of stages in development of sea urchin, from unfertilized egg to pluteus larvae. 


\section{Sediment Pore-Water Toxicity Test Results and Preliminary Toxicity Identification of Post-Landfall Pore-Water Samples Collected Following the Deepwater Horizon Oil Release, Gulf of Mexico, 2010}

By James M. Biedenbach and Robert S. Carr

Open-File Report 2011-1078 


\section{U.S. Department of the Interior \\ KEN SALAZAR, Secretary \\ U.S. Geological Survey \\ Marcia K. McNutt, Director}

\section{U.S. Geological Survey, Reston, Virginia: 2011}

For more information on the USGS — the Federal source for science about the Earth, its natural and living resources, natural hazards, and the environment, visit http://www.usgs.gov or call 1-888-ASK-USGS.

For an overview of USGS information products, including maps, imagery, and publications, visit http://www.usgs.gov/pubprod

Any use of trade, product, or firm names is for descriptive purposes only and does not imply endorsement by the U.S. Government.

Although this report is in the public domain, permission must be secured from the individual copyright owners to reproduce any copyrighted materials contained within this report.

Suggested citation:

Biedenbach, J.M., Carr, R.S., 2011, Sediment pore-water toxicity test results and preliminary toxicity identification of post-landfall pore-water samples collected following the Deepwater Horizon oil release, Gulf of Mexico, 2010: U.S. Geological Survey Open-File Report 2011-1078, 79 p. 


\section{Acknowledgments}

The authors thank Dr. Marion Nipper, Dr. Kim Withers, and Misti Grohman of the Texas A\&M University-Corpus Christi Center for Coastal Studies, for their assistance in completing the toxicity testing and water-quality analysis.

The authors also thank Suzanne Roberts of the U.S. Geological Survey for her assistance in creating the figures. 


\section{Contents}

Acknowledgments ....................................................................................................................ii

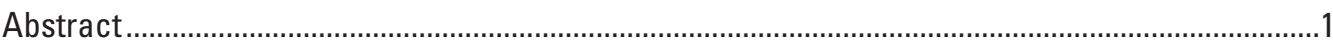

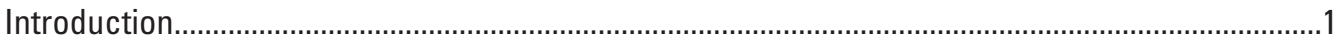

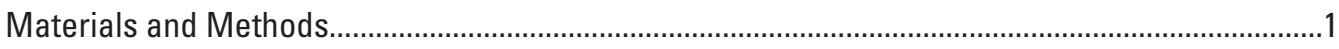

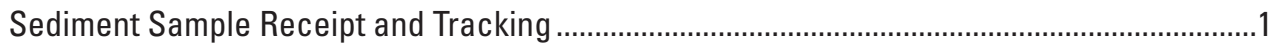

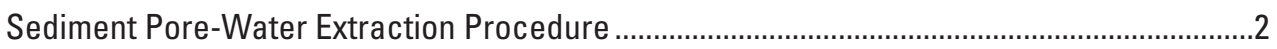

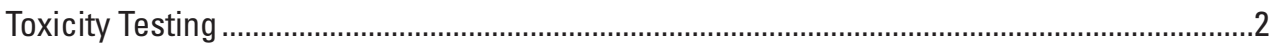

Pore-Water Quality Measurements and Salinity Adjustment ...........................................2

Pre-Landfall and Post-Landfall Toxicity Testing with Sea Urchins....................................2

Toxicity Identification Evaluation Toxicity Testing with Sea Urchins ..................................3

Sea Urchin Toxicity Testing Data Analysis ..........................................................................

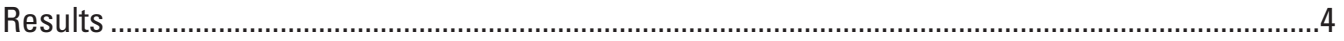

Pre-Landfall Pore-Water-Quality Measurements ................................................................

Pre-Landfall Sea Urchin Toxicity Testing ..............................................................................

Post-Landfall Pore-Water-Quality Measurements...................................................................

Post-Landfall Sea Urchin Toxicity Testing ........................................................................

Toxicity Identific ation Evaluation Pore-Water-Quality Measurements ......................................5

Toxicity Identification Evaluation Sea Urchin Toxicity Testing .................................................

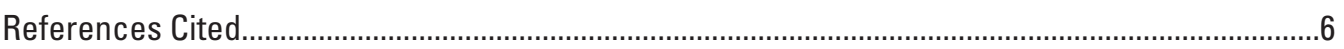

Appendix 1. Extraction and Storage of Pore-Water Samples .......................................................36

Appendix 2. Sea Urchin Fertilization Toxicity Test.......................................................................46

Appendix 3. Sea Urchin Embryological Development Toxicity Test ................................................62

\section{Figures}

1. Map showing sample sites in Texas, Louisiana, Mississppi, Alabama and Florida and locations of laboratories.

2. Map showing sample sites in Texas and Louisiana and toxicity test results of the sea urchin fertilization and embryological development post-landfall tests of 100-, 50- and 25-percent salinity adjusted pore water.

3. Map showing sample sites in Mississippi and Alabama and toxicity test results of the sea urchin fertilization and embryological development post-landfall tests of 100-, 50- and 25-percent salinity adjusted pore water

4. Map showing sample sites in Florida and toxicity test results of the sea urchin fertilization and embryological development post-landfall tests of $100-$, 50- and 25-percent salinity adjusted pore water.

\section{Appendix 1}

1-1. Schematic of sediment pore water squeeze extraction device 37

1-2. Schematic of sediment pore-water pressure extraction system 


\section{Appendix 3}

3-1. Artist's sketches of stages in development of sea urchin, from unfertilized egg to pluteus larvae...

\section{Tables}

1. Relevant sample and extraction dates, times, temperatures and volumes of pore water collected from sediments pre-landfall of the Deepwater Horizon oil release.

2. Relevant sample and extraction dates, times, temperatures and volumes of pore water collected from sediments post-landfall of the Deepwater Horizon oil release.

3. Water-quality measurements after salinity adjustment and original salinity of pore-water samples collected from sediments pre-landfall of the Deepwater Horizon oil release

4. Sea urchin fertilization test raw data and means for pore-water samples collected from sediments pre-landfall of the Deepwater Horizon oil release

5. Sea urchin embryological development test raw data and means for pore-water samples collected from sediments pre-landfall of the Deepwater Horizon oil release.

6. Water-quality measurements after salinity adjustment and original salinity of pore-water samples collected from sediments post-landfall of the Deepwater Horizon oil release.

7. Sea urchin fertilization test raw data and means for experiment one with pore-water samples collected from sediments post-landfall of the Deepwater Horizon oil release.

8. Sea urchin fertilization test raw data and means for experiment two with pore-water samples collected from sediments post-landfall of the Deepwater Horizon oil release

9. Sea urchin embryological development raw data and means for pore-water samples collected from sediments post-landfall of the Deepwater Horizon oil release.

10. Water-quality measurements after salinity adjustment and original salinity of pore-water samples collected from sediments post-landfall of the Deepwater Horizon oil release and tested in the Toxicity Identification Evaluation.

11. Toxicity Identification evaluation fertilization raw data ans means for porewater samples identified as toxic in the Deep Water Horizon post-landfall sea urchin fertilization test.

12. Toxicity Identification evaluation embryological development raw data and means for pore-water samples identified as toxic in the Deep Water Horizon post-landfall sea urchin embryological development test 


\section{Conversion Factors}

SI to Inch/Pound

\begin{tabular}{lcl}
\hline \multicolumn{1}{c}{ Multiply } & \multicolumn{1}{c}{ By } & \multicolumn{1}{c}{ To obtain } \\
\hline liter $(\mathrm{L})$ & Volume & \\
liter $(\mathrm{L})$ & 33.82 & ounce, fluid (fl. oz) \\
liter $(\mathrm{L})$ & 2.113 & pint (pt) \\
liter $(\mathrm{L})$ & 1.057 & quart (qt) \\
liter $(\mathrm{L})$ & 0.2642 & gallon (gal) \\
milliliters $(\mathrm{mL})$ & 61.02 & cubic inch (in $\left.{ }^{3}\right)$ \\
microliters $(\mu \mathrm{L})$ & 0.3382 & ounce, fluid (fl. oz) \\
& 0.00003382 & ounce, fluid (fl. oz) \\
\hline gram $(\mathrm{g})$ & Mass & ounce, avoirdupois $(\mathrm{oz})$ \\
milligrams $(\mathrm{mg})$ & 0.03527 & ounce, avoirdupois $(\mathrm{oz})$ \\
micrograms $(\mu \mathrm{g})$ & 0.00003527 & ounce, avoirdupois $(\mathrm{oz})$ \\
\hline & 0.00000003527 & \\
\hline micrometer $(\mu \mathrm{m})$ & Length & inches (in) \\
\hline
\end{tabular}

Temperature in degrees Celsius $\left({ }^{\circ} \mathrm{C}\right)$ may be converted to degrees Fahrenheit $\left({ }^{\circ} \mathrm{F}\right)$ as follows:

${ }^{\circ} \mathrm{F}=\left(1.8 x^{\circ} \mathrm{C}\right)+32$

Concentrations of chemical constituents in water are given either in milligrams per liter (mg/L) or micrograms per liter $(\mu \mathrm{g} / \mathrm{L})$.

Salinities are given in parts per thousand $(\%)$ equivalent to grams per liter $(\mathrm{g} / \mathrm{L})$. 


\title{
Sediment Pore-Water Toxicity Test Results and Preliminary Toxicity Identification of Post-Landfall Pore-Water Samples Collected Following the Deepwater Horizon Oil Release, Gulf of Mexico, 2010
}

\author{
By James M. Biedenbach and Robert S. Carr
}

\begin{abstract}
Pore water from coastal beach and marsh sediments from the northern Gulf of Mexico, pre- and post-landfall of the Deepwater Horizon oil release, were collected and evaluated for toxicity with the sea urchin fertilization and embryological development assays. There were 17 pre-landfall samples and 49 post-landfall samples tested using both assays. Toxicity was determined in four pre-landfall sites and in seven post-landfall sites in one or both assays as compared to a known reference sediment pore-water sample collected in Aransas Bay, Texas. Further analysis and testing of five of the post-landfall toxic samples utilizing Toxicity Identification Evaluation techniques indicated that ammonia, and to a lesser extent metals, contributed to most, if not all, of the observed toxicity in four of the five samples. Results of one sample (MS-39) indicated evidence that ammonia, metals, and non-ionic organics were contributing to the observed toxicity.
\end{abstract}

\section{Introduction}

On April 20, 2010, the Deepwater Horizon oil rig exploded and collapsed to the sea floor resulting in the Nation's largest documented oil release to the marine environment (Crone and Tolstoy, 2010). The U.S. Geological Survey (USGS) was commissioned by the U.S. Coast Guard Unified Area Command to assess any onshore effects related to the release and the subsequent cleanup. Sediment and water samples from beaches and wetlands were collected along the coast from Florida to Texas with a focus on those areas that were anticipated to be affected or were affected by oil coming to shore (fig. 1, at the back of the report). USGS scientists were tasked with identifying and fingerprinting oil in the sediment and dispersants in the water applied in the response effort, and determining the severity of the toxicity of surficial sediment collected from the study area (Rosenbauer and others, 2010).
Toxicity was assessed using sediment pore water in the sea urchin (Arbacia punctulata) fertilization and embryological development tests. A secondary effort also was initiated to characterize the observed toxicity indicated in the post-landfall samples. Sediment samples were collected by USGS scientists in each of the five affected States in May and June, 2010, before the oil making landfall, and in October 2010, following landfall and subsequent cleanup in some areas. Sediment samples were shipped to the USGS in Corpus Christi, Texas for toxicity testing (fig. 1).

The specific objectives of this study were to:

- Extract sediment pore water from sediment samples collected pre- and post-landfall from areas of possible ecological effects in Florida, Alabama, Mississippi, Louisiana and Texas (fig. 1).

- Measure water-quality parameters (salinity, dissolved oxygen, $\mathrm{pH}$, sulfide, and ammonia) of pore-water samples before toxicity testing.

- Conduct the fertilization and embryological development toxicity tests with pore water using sea urchin (A. punctulata) gametes.

- Perform preliminary phase I Toxicity Identification Evaluation (TIE) toxicity testing with toxic post-landfall samples to determine the class of contaminant(s) contributing to the toxicity.

\section{Materials and Methods}

\section{Sediment Sample Receipt and Tracking}

Surficial sediment samples were collected from 70 sites along the Florida, Alabama, Mississippi, Louisiana, and upper Texas coasts in May and June 2010 before the Deepwater Horizon oil making landfall. Of the 86 sediment samples 
received at USGS, 83 had sufficient sediment volume and moisture to be extracted for pore water. Extended holding times and unacceptable temperatures during shipment resulted in 17 of those samples (representing 15 sites) meeting the criteria for quality control; therefore, only those 17 samples were tested in the pre-landfall sampling round (table 1, at the back of the report). The limited volume of some samples ( 2 of 17) did not allow for a complete dilution series; therefore, a reduced number of replicates were tested in these samples.

In October 2010, surficial sediment samples were resampled at 49 sites along the Gulf Coast. These 49 samples were collected using more rigorous protocol developed during summer 2010. A single site was collected in August 2010, to test the implementation of the new protocol and was included in the pore-water extraction and post-landfall testing. Despite variations in the sampling protocols between pre- and postlandfall, toxicity results are comparable as all tested samples were collected with inert materials and met the quality control criteria upon arrival at USGS. Samples were placed in precleaned 1 liter glass containers, chilled, and shipped overnight by FedEx ${ }^{\circledR}$ in insulated coolers with bagged ice to USGS. Shipments were accompanied by chain of custody sheets, were recorded into a log book, and incoming temperatures were recorded for each container with a Fisher-Scientific Traceable ${ }^{\circledR}$ $\mathrm{Ultra}^{\mathrm{TM}}$ waterproof thermometer with probe/cable. All postlandfall pore-water samples were extracted within 4 days from the time of field collection of sediment, and within 12 hours of arrival at the USGS laboratory (table 2, at the back of the report).

\section{Sediment Pore-Water Extraction Procedure}

Pore-water volumes ranging from 40 to 450 milliliters were extracted from each sediment sample using a pneumatic extraction device (Carr and Chapman, 1992; 1995; Carr and others, 1996a, 1996b; U.S. Geological Survey, 2007). The extractor is made of polyvinyl chloride (PVC) and uses a 5-micrometer $(\mu \mathrm{m})$ polyester filter mesh. The apparatus and extraction procedures are detailed in CERC SOP P.649 (appendix 1). After extraction, the pore-water samples were centrifuged in polycarbonate bottles at 1,200 times gravity for 20 minutes to remove any suspended particulate material; the supernatant was collected, and stored in precleaned samples bottles (from one to three depending on the volume collected), and frozen at negative 11 plus or minus $( \pm) 1$ degree Celsius $\left({ }^{\circ} \mathrm{C}\right)$.

\section{Toxicity Testing}

\section{Pore-Water Quality Measurements and Salinity Adjustment}

Two days before conducting a toxicity test, pore-water samples were allowed to partially thaw at room temperature for 4 hours before being moved to a refrigerator kept at
$4{ }^{\circ} \mathrm{C}$. One day before testing, samples were brought to room temperature in a tepid $\left(20 \pm 2{ }^{\circ} \mathrm{C}\right)$ water bath for 1 hour. Sample salinity was measured and adjusted to $30 \pm 1$ parts per thousand (\%o), if necessary using Milli- ${ }^{\circledR}$ purified de-ionized water or concentrated brine (102\%). Following any salinity adjustments, the samples were stored overnight at $4{ }^{\circ} \mathrm{C}$, but were returned to $20 \pm 1{ }^{\circ} \mathrm{C}$ (incubated in an environmental chamber) immediately before the start of the toxicity tests. On the day of the test, subsamples were collected, acclimated to room temperature, and measured for water-quality parameters (dissolved oxygen, $\mathrm{pH}$, and ammonia). Additional subsamples were preserved with Sulfide Anti-oxidant Buffer (SAOB II ${ }^{\circledR}$ ) reagent for sulfide measurements (Thermo Fisher Scientific Inc., 2007) and refrigerated to $4{ }^{\circ} \mathrm{C}$ until the following day, when they were brought to room temperature and measured. Dissolved oxygen (DO) concentrations were measured with a Yellow Springs Instruments Incorporated $\left(\mathrm{YSI}^{\circledR}\right)$ model 59 dissolved oxygen meter with a YSI ${ }^{\circledR}$ model 5905 Biological Oxygen Demand (BOD) probe. Salinity was measured with a Reichert ${ }^{\circledR}$ temperature compensated refractometer. Sulfide (as $\mathrm{S}^{-2}$ ), $\mathrm{pH}$, and total ammonia (expressed as total ammonia nitrogen; TAN) were measured with Orion ${ }^{\circledR}$ model 290 A meters and the Thermo-Orion ${ }^{\circledR}$ model 9616 silver/sulfide probe, Thermo-Orion ${ }^{\circledR}$ model 9107BN low maintenance triode, and the Thermo-Orion ${ }^{\circledR}$ model 5912 ammonia probe, respectively. Room and environmental chamber temperature were measured using a Fisher-Scientific Traceable ${ }^{\circledR}$ Ultra $^{\mathrm{TM}}$ Waterproof Thermometer with probe/cable calibrated to National Institute of Standards and Technology (NIST) standards. Un-ionized ammonia (expressed as un-ionized ammonia nitrogen; UAN) concentrations were calculated for each sample using the respective salinity, temperature, $\mathrm{pH}$, and TAN values (Bowers and Bidwell, 1978). No samples had dissolved oxygen levels below 80 percent saturation; therefore, aeration was not conducted.

\section{Pre-Landfall and Post-Landfall Toxicity Testing with Sea Urchins}

Sea urchins (A. punctulata) were obtained from Gulf Specimens Marine Laboratories, Inc., Panacea, Florida (fig. 1). For fertilization and embryological development tests, each pore-water sample with adequate volume was tested in a dilution series at 100, 50, and 25 percent of the sample (after salinity adjustment) with five replicates per dilution treatment (CERC SOP P.647, appendix 2, and CERC SOP P.648, appendix 3). A reduced number of replicates or removal of dilutions was implemented in cases where sample volume was limited.

A pretest was conducted to determine the optimum sperm dilution that would give acceptable fertilization rates in the reference pore water, dilution water, and reference toxicant dilutions. All pretests and fertilization tests were conducted at $20^{\circ} \mathrm{C}$ in a temperature controlled chamber, and an exposure time of 30 minutes each for the sperm and the sperm plus eggs. At the end of the exposure, the tests were terminated by 
the addition of buffered formalin. The presence or absence of a fertilization membrane was determined by examining 100 eggs/replicate using a compound microscope. The percent fertilization was determined using the following formula:

Total Number of Eggs - Number of Eggs Unfertilized Total Number of Eggs

The embryological development test was performed in a similar manner to the fertilization test, except that the eggs were fertilized before being added to the sample vials. The test was terminated after 48 hours by the addition of buffered formalin. The percent normally developed pluteus stage larvae was determined by microscopic examination.

Dilutions were made with $0.45-\mu \mathrm{m}$ Millipore ${ }^{\circledR}$ filtered seawater (MFS). A reference pore-water sample was collected from Aransas Bay, Texas, (fig. 1) using a PVC corer, refrigerated $\left(4^{\circ} \mathrm{C}\right)$, and extracted identically to the test samples, as a negative toxicity control. This reference sediment was collected on October 20, 2010, and held refrigerated and processed for pore water on October 21,2010. This site is far removed from any known sources of contamination and has been used previously as a reference site (U.S. Geological Survey, 2007). In addition, a dilution water blank of filtered seawater was included in each test and a brine blank (control pore water diluted to $6 \%$ with Milli- ${ }^{\circledR}$ purified water and subsequently increased with brine) was included in those tests that contained samples that were adjusted with brine or Milli-Q ${ }^{\circledR}$ water. A rinsate blank also was included in each initial post-landfall test, which consisted of MFS put through all the extraction equipment, and centrifuged just as a sediment sample had been processed to determine if there was contamination from any cleaning procedure. Finally, a reference toxicity test (positive control sample) was conducted in each assay with a dilution series of sodium dodecyl sulfate (SDS) to evaluate overall test sensitivity.

\section{Toxicity Identification Evaluation Toxicity Testing with Sea Urchins}

Sites determined to be toxic in the sea urchin fertilization or embryological development test were analyzed further using TIE procedures to attempt to characterize the type of contaminant(s) contributing to the observed toxicity. The procedures used to perform the TIE are described by the United States Environmental Protection Agency (U.S. Environmental Protection Agency, 1996). Because of the limited sample volume remaining, four sample manipulations were selected to characterize the toxicity of each sample, and no dilutions or $\mathrm{pH}$ manipulations were performed. The sample manipulations included the addition of Disodium Ethylenediamine Tetraacetate (EDTA) at a concentration of $60 \mathrm{mg} / \mathrm{L}$ to chelate metals; vigorous aeration to drive off volatile organics and hydrogen sulfide; $\mathrm{C}_{18}$ solid phase extraction (SPE) to remove non-ionic organic compounds; and exposure to the algae Ulva lactuca $(0.083$ grams per milliliter $(\mathrm{g} / \mathrm{mL})$ to adsorb ammonia (U.S. Environmental Protection Agency, 1996).

EDTA was added to a subsample of each pore-water sample by first preparing a 25 grams per liter $(\mathrm{g} / \mathrm{L})$ EDTA stock solution and adding 12 microliters $(\mu 1)$ per 5 milliliters $(\mathrm{mL})$ of sample volume contained in a beaker, and, incubating at $20^{\circ} \mathrm{C}$ for 3 hours before testing. Following incubation, samples were pipetted into vials for testing.

Six mL Bakerbond ${ }^{\mathrm{TM}}$ SPE Octadecyl $\left(\mathrm{C}_{18}\right)$ disposable extraction columns containing $1,000 \mathrm{mg}$ of resin were used to remove active non-ionic organic contaminants. A separate column was used for each sample, and each was activated with $10 \mathrm{~mL}$ of methanol and rinsed with MFS before receiving the sample. Samples were pushed through the columns with a glass syringe into glass beakers, and subsequently pipetted into test vials.

Vigorous aeration was accomplished with an aquarium pump attached to a gang valve manifold to which Pasteur pipettes were attached and placed in beakers containing the samples. Samples were pipetted into test vials after an hour of aeration. Subsamples were collected and preserved for analysis of sulfide reduction.

Ulva lactuca were collected from the jetties in Port Aransas, Texas (fig. 1) the day before testing. Plants were sorted and subsamples weighed $(0.083 \mathrm{~g} / \mathrm{mL})$ for each treatment sample and placed in large beakers with MFS and gentle aeration. Ulva lactuca aliquots were kept incubated in the dark at $15^{\circ} \mathrm{C}$ overnight before the test. On the test day, each algae aliquot was removed from its beaker, carefully dried with a lint free towel, and placed into the beaker containing the porewater sample. The samples were then incubated at $20^{\circ} \mathrm{C}$ for 5 hours with gentle aeration. At the end of incubation, the pore water was pipetted out of the each beaker into the test vials. Pore-water subsamples were collected and ammonia measurements performed as described previously to determine the extent of ammonia removal.

Each of the five samples was treated with all four manipulations and compared against their untreated counterpart to determine the effect of the manipulation on toxicity. A nontoxic pore-water sample extracted from an Aransas Bay, Texas, sediment served as a blank, and was treated the same as the test samples to determine if any of the treatment manipulations produced toxic artifacts. A brine blank and SDS dilution series also were included to gage overall test sensitivity. Either the fertilization or embryological development test was used for each sample. The test selected was based on the greatest toxic response achieved in the post-landfall testing. An ammonia dilution series $\left(\mathrm{NH}_{4} \mathrm{Cl}\right)$ in MFS was included in the Toxicity Identification Evaluation testing to determine the sensitivity of this batch of sea urchin embryos to ammonia. The un-ionized ammonia fraction was calculated using the measured TAN, salinity $(30 \%)$, temperature $\left(20^{\circ} \mathrm{C}\right)$ and the $\mathrm{pH}$ of the individual concentrations measured before adding fertilized eggs to the sample vials (Bowers and Bidwell, 1978). 


\section{Sea Urchin Toxicity Testing Data Analysis}

Detectable significance criteria (DSC) have been developed to determine the 95-percent confidence limit based on power analysis of similar tests performed by the USGS lab (Carr and Biedenbach, 1999). The DSC value for the sea urchin fertilization assay at $\alpha=0.05$ is 15.5 percent. At $\alpha=0.01$, the DSC value is 19 percent. The DSC values for the sea urchin embryological development tests are 16.4 percent and 20.6 percent for $\alpha=0.05$ and 0.01 , respectively. The DSC is applied by multiplying the DSC percentage by the reference mean, and subtracting that value from the reference mean to obtain a cut-off value. Results below this value are considered toxic. For instance, the sea urchin fertilization DSC cut-off value at $\alpha=0.05$ for a reference mean of 95 percent would be 80.3 percent [ $(95-(0.155 \times 95)]$. The trimmed Spearman-Karber method (Hamilton and others, 1977) with Abbott's correction (Morgan, 1992) was used to calculate 50-percent effective concentration $\left(\mathrm{EC}_{50}\right.$ ) values for the SDS and ammonia dilution series.

For the TIE tests, statistical comparisons between the treated and untreated pore water were made using Analysis of Variance (ANOVA) and Dunnett's one-tailed $t$-test (controls the experiment-wise error rate) on the arcsine square root transformed data with the aid of SAS (SAS, 1989). Replicates were removed only from statistical consideration if there were known errors in the pipetting of the gametes to the vials.

\section{Results}

\section{Pre-Landfall Pore-Water-Quality Measurements}

Water-quality measurements were conducted on sediment pore water from 15 sites plus 2 duplicates of 1 of the sites (LA-23; table 3, at the back of the report). Salinity adjustment with Milli- $\mathrm{Q}^{\circledR}$ purified de-ionized water was required with 4 samples, whereas 12 samples and the reference control required adjustments with brine. Salinities in test samples ranged from nearly fresh water at $0.5 \%$ to greater than full strength seawater at $40 \%$. Initial dissolved oxygen was greater than 80 percent in all the samples. Total ammonia ranged from less than 0.1 to $5.97 \mathrm{mg} / \mathrm{L}$, whereas the un-ionized ammonia (the most toxic fraction) ranged from less than 3.3 to $214.8 \mu \mathrm{g} / \mathrm{L}$. There were four samples (FL-11 Rep 2, FL-13, FL-14 and FL-26; table 3) that approached or exceeded the $\mathrm{EC}_{50}$ for un-ionized ammonia (48.79 $\mu \mathrm{g} / \mathrm{L}$; 95 -percent confidence limits $47.53-50.08 \mu \mathrm{g} / \mathrm{L}$ ) calculated for the ammonia dilution series included with the TIE testing. This level is slightly lower than what has been previously reported for this species (Winger and others, 2003). Detectable sulfide concentrations were measured in only one pore-water sample (FL-11 Rep 2; table 3) at the detection limit of $0.009 \mathrm{mg} / \mathrm{L}$.

\section{Pre-Landfall Sea Urchin Toxicity Testing}

A fertilization test and an embryological development test were conducted with the same sea urchin gametes as part of the sea urchin toxicity testing (tables 4 and 5, at the back of the report). Two of the 17 samples had reduced volume and, therefore, had a reduced number of replicates, dilutions, or both in the tests. Eggs from three females were combined to achieve the necessary quantity required for the tests. Sperm from one male was used in both tests. The $\mathrm{EC}_{50}$ value for the SDS positive control for the fertilization test was calculated at $4.18 \mathrm{mg} / \mathrm{L}$. This value is similar to the historical mean for this species for the USGS laboratory of $5.43 \mathrm{mg} / \mathrm{L}$ (95-percent confidence limits 3.20 to $7.66 \mathrm{mg} / \mathrm{L}$ ) and indicates adequate test sensitivity. A significant difference from the reference control in the fertilization test (FL-11 Rep 2, FL-26 and LA-29) was indicated in 3 of the 17 pore-water samples; however, none met the DSC cut-off of 83.4 percent for this test (table 4). The $\mathrm{EC}_{50}$ value for the SDS positive control for the embryological development test was calculated at $3.66 \mathrm{mg} / \mathrm{L}$ (3.47$3.87 \mathrm{mg} / \mathrm{L}$ ), which is slightly below the historical mean of $4.24 \mathrm{mg} / \mathrm{L}$ (95-percent confidence limits 1.68 to 6.80 ) for the USGS laboratory, but is within the accepted confidence intervals. Embryological development results from four samples (FL-11 Rep 2, FL-13, FL-14 and FL-26; table 5) were below the DSC cut-off criteria of 71.9 percent for the 100 percent salinity-adjusted pore-water concentration. These four samples also had un-ionized ammonia levels that approached or exceeded the $\mathrm{EC}_{50}$ value $(48.79 \mu \mathrm{g} / \mathrm{L}$; 95 -percent confidence limits $47.53-50.08 \mu \mathrm{g} / \mathrm{L}$ ) calculated for the ammonia series. Sample FL-14 also was below the DSC cut-off values at the 50- and 25-percent pore-water concentrations.

\section{Post-Landfall Pore-Water-Quality Measurements}

Water-quality measurements were conducted on sediment pore water from 49 sites, as well as the reference, dilution water, brine, and rinsate blanks (table 6 , at the back of the report). Salinity adjustment with Milli-Q ${ }^{\circledR}$ purified de-ionized water was required for 17 pore-water samples, whereas 26 pore-water samples and the reference control required adjustments with brine. Salinities in test samples ranged from nearly fresh water at $1 \%$ to full strength seawater at $35 \%$. Sample LA-26 contained suspended particulates after thawing and water-quality measurement, and required centrifugation before testing to eliminate suspended particles that could interfere with fertilization. Total ammonia ranged from less than 0.1 to $0.793 \mathrm{mg} / \mathrm{L}$, whereas the un-ionized ammonia (the most toxic fraction) ranged from less than 1.9 to $48.9 \mu \mathrm{g} / \mathrm{L}$. Sulfide concentrations were measured in 14 pore-water samples, and ranged from less than detection at $0.009 \mathrm{mg} / \mathrm{L}$ to as much as $0.048 \mathrm{mg} / \mathrm{L}$. 


\section{Post-Landfall Sea Urchin Toxicity Testing}

On October 26, 2010, three tests were conducted with the same sea urchin gametes; two for the fertilization test, and one for the embryological development test (tables 7-9, at the back of the report). Eggs from four females were combined to achieve the necessary quantity required for the three tests. Sperm from one male was used in all three tests. The $\mathrm{EC}_{50}$ value for the SDS positive controls for the fertilization tests were calculated at 5.47 and $5.63 \mathrm{mg} / \mathrm{L}$ for tests one and two, respectively. These values are similar to the historical mean for this species for the USGS laboratory of $5.43 \mathrm{mg} / \mathrm{L}$ (95-percent confidence limits 3.20 to $7.66 \mathrm{mg} / \mathrm{L}$ ) and indicate adequate test sensitivity. Only three pore-water samples indicated a significant difference from the reference control in the fertilization tests using the DSC (figs. 2-4, at the back of the report). Fertilization percentages from samples from sites LA-25, LA-29, and LA-36 (fig. 2) were below the DSC cut-off value of 81.9 percent for the 100-percent concentration of the pore water in the first test. Site LA-29 (fig. 2) also was significantly different in the 50- and 25-percent diluted pore-water samples. No sample means were less than the DSC cut-off value of 81.2 percent in the second fertilization test.

Raw data and means from the embryological development test are reported in table 9. The $\mathrm{EC}_{50}$ value for the SDS positive control for the embryological development test was calculated at $3.44 \mathrm{mg} / \mathrm{L}(3.28-3.60 \mathrm{mg} / \mathrm{L})$, which is slightly below the historical mean of $4.24 \mathrm{mg} / \mathrm{L}$ (95-percent confidence limits 1.68 to $6.80 \mathrm{mg} / \mathrm{L}$ ) for the USGS laboratory but was within the accepted 95-percent confidence intervals. Development results from six samples (LA-25, LA-26, LA-31, LA-33, LA-36, and MS-39) were below the DSC cut-off of 78.3 percent for the 100 -percent salinity adjusted pore-water concentration (figs. 2-4). No samples were below the DSC at the 50- and 25-percent pore-water concentrations. Notably, samples from sites LA-26 and LA-31 had the greatest ammonia and sulfide concentrations of the samples collected and tested in the post-landfall round of testing.

\section{Toxicity Identification Evaluation Pore-Water- Quality Measurements}

After testing of the post-landfall samples, seven samples (LA-25, LA-26, LA-29, LA-31, LA-33, LA-36, and MS-39) were determined to be toxic in either the fertilization or embryological development test based on the exceedance of the DSC. The remaining volume of pore water in frozen storage was sufficient for five samples (all but LA-25 and LA-26) to perform a partial phase I TIE to characterize the toxicity observed. Water-quality measurements were conducted on the five test pore water samples and the reference pore water, as well as the dilution water and a brine blank (table 10, at the back of the report). Initial salinities ranged from 6 to $29 \%$ for the pore-water samples. Total ammonia concentration was greater overall for this set of samples than from the concentration measured during the post-landfall testing conducted in October 2010, indicating a possible problem with the October measurements. Total ammonia concentrations ranged from 0.903 to $2.72 \mathrm{mg} / \mathrm{L}$ for the test samples (table 10 ). Samples LA-31 $(94.7 \mu \mathrm{g} / \mathrm{L})$ and LA-33 $(92.0 \mu \mathrm{g} / \mathrm{L})$ had unionized ammonia values that exceeded the $\mathrm{EC}_{50}(49.79 \mu \mathrm{g} / \mathrm{L})$ for toxicity for the sea urchin embryological development test determined in the ammonia series included in this test. Measurable amounts of ammonia were recorded for all the samples initially tested (table 10). Following Ulva latuca treatment, a reduction in ammonia levels was observed in every sample to below the detection limit of $0.1 \mathrm{mg} / \mathrm{L}$. Sulfide was measured in two samples (LA-29 and LA-33; table 10) above the detection level of $0.009 \mathrm{mg} / \mathrm{L}$ and was comparable to measurements made in the October 2010, post-landfall testing; however, measurements made after aeration indicated little change in sulfide concentration. Sample LA-29 decreased from a reading of $0.04 \mathrm{mg} / \mathrm{L}$ to $0.033 \mathrm{mg} / \mathrm{L}$, whereas sample LA-33 virtually was unchanged after aeration. This indicates that the aeration treatment was not effective in removing the sulfide, and may complicate interpretation of results in those samples containing toxic concentrations of sulfide.

\section{Toxicity Identification Evaluation Sea Urchin Toxicity Testing}

Sample LA-29 was tested with its various treatment manipulations within the sea urchin fertilization assay (table 11, at the back of the report). The initial untreated porewater sample exhibited similar toxicity to that documented in the October 2010, post-landfall testing. Of the four treatments, only two had any effect on reducing toxicity to the sample; the addition of EDTA had a small but significant effect raising the mean from 13 percent fertilized to 22.2 percent fertilized. The largest reduction (from 13 percent to 87.3 percent) was observed in the Ulva lactuca treatment, as all of the toxicity was removed. This indicates that ammonia with some minor metals contribution is contributing to most of the observed toxicity; however, this is somewhat surprising because the fertilization test is not sensitive to ammonia toxicity when compared to the embryological development assay (Winger and others, 2003), and this sample was not determined to be toxic in the embryological development assay. Sulfides or other related compounds may be playing a role in that they were not removed by the aeration treatment, but may have been taken up by the Ulva lactuca treatment. The lack of increased fertilization of the sample treated with the $\mathrm{C}_{18}$ column indicates that organics probably are not a primary contributing factor to the toxicity. Treatment blanks with the non-toxic Texas pore water indicated no artifacts imparted to the samples from the various manipulations.

The remaining four samples with their respective treatment manipulations were tested with the sea urchin embryological development test (table 12, at the back of the report). Untreated sample LA-31 exhibited toxicity similar to that 
previously measured in the post-landfall assessment. Only the Ulva lactuca treatment was successful in reducing toxicity to the level of nontoxic. This indicates ammonia was the primary contaminant and, indeed, the un-ionized ammonia measurement was nearly double the $\mathrm{EC}_{50}$ for toxicity determined in the ammonia series included in this test. No other sample manipulation was significant in changing the toxicity.

Untreated sample LA-33 also exhibited toxicity similar to that previously measured in the October 2010 post-landfall assessment. As with sample LA-29, Ulva latuca treatment reduced the toxicity completely, whereas EDTA addition also reduced the toxicity level to a lesser but significant extent. $\mathrm{C}_{18}$ treatment also seemed to reduce the toxicity level slightly, but was not determined to be significant. This indicates that ammonia and metals contributed to the observed toxicity. The un-ionized ammonia level in this sample $(92 \mu \mathrm{g} / \mathrm{L})$ also was well above the $\mathrm{EC}_{50}$ for toxicity determined for this test, indicating that it may have been the primary stressor in sample LA-33.

Sample LA-36 exhibited a reduced toxic response in the untreated sample compared to previous testing in the postlandfall assessment. Although still toxic, the lower response makes it more difficult to discern the effects of the sample manipulations on the level of toxicity; however, two treatments did make an improvement to the level of toxicity. The Ulva lactuca treatment and the EDTA addition reduced the toxicity to zero indicating that metals and ammonia are contributing to the observed toxicity. The aeration treatment significantly increased the toxicity of the sample indicating that the sample was evaporating and concentrating the contaminants.

Sample MS-39 exhibited slightly reduced toxicity in the untreated sample compared to the initial testing in the October post-landfall assessment but still had a fairly strong signal. As with the other samples, Ulva lactuca treatment removed all of the toxicity and EDTA addition also removed a significant part of it. This sample differed from the others in that the $\mathrm{C}_{18}$ treatment also removed nearly all of the toxicity from the sample. Although the measured amount of un-ionized ammonia was not great enough to cause toxicity, the Ulva lactuca treatment may have been taking up non-ionic toxicants as well as ammonia. Ulva lactuca is known to selectively take up some non-ionic organic toxicants as well as ammonia (Ho and others, 1999). $\mathrm{C}_{18}$ is not known to uptake ammonia, but it will chelate some metals such as copper (U.S. Environmental Protection Agency, 1996). It is possible that ammonia, metals, and non-ionic organics are contributing to the observed toxicity of this sample.

\section{References Cited}

Bowers, C.E., and Bidwell, J.P., 1978, Ionization of ammonia in seawater-Effects of temperature, $\mathrm{pH}$, and salinity: Journal of the Fisheries Research Board of Canada, v. 35, p. 1,012-1,016.

Carr, R.S., and Biedenbach, J.M., 1999, Use of power analysis to develop detectable significance criteria for sea urchin toxicity tests: Aquatic Ecosystem Health. Management, v. 2, p. 413-418.

Carr, R.S., and Chapman, D.C., 1992, Comparison of solidphase and pore-water approaches for assessing the quality of marine and estuarine sediments: Journal of Chemical Ecology, v. 7, p. 19-30.

Carr, R.S., and Chapman, D.C., 1995, Comparison of methods for conducting marine and estuarine sediment porewater toxicity tests - Extraction, storage, and handling techniques: Archives of Environmental Contamination and Toxicology, v. 28 , p. $69-77$.

Carr, R.S., Long, E.R., Windom, H.L., Chapman, D.C., Thursby, G., Slone, G.M., and Wolfe, D.A., 1996a, Sediment Quality Assessment Studies of Tampa Bay, Florida: Environmental Toxicology and Chemistry, v. 15, p. 1,218-1,231.

Carr, R.S., Chapman, D.C., Howard, C.L., and Biedenbach, J.M., 1996b, Sediment quality triad assessment survey of the Galveston Bay, Texas system: Ecotoxicology, v. 5, p. 341-364.

Crone, T.J., and Tolstoy, M., 2010, Magnitude of the 2010 Gulf of Mexico oil leak: Science, v. 330, 634 p.

Hamilton, M.A., Russo, R.C., and Thurston, R.V., 1977, Trimmed Spearman-Karber method for estimating median lethal concentrations in toxicity bioassays: Environmental Science and Technology, v. 11, p.714-719; Correction v. 12, p. 417 (1978).

Ho, K.T., Kuhn, A., Pelletier, M.C., Burgess, R.M., and Helmsletter, A., 1999, Use of Ulva lactuca to distinguish $\mathrm{pH}$-dependant toxicants in marine waters and sediments: Environmental Toxicology and Chemistry, v. 18, no. 2, p. 207-212. 
Morgan, B.J.T., 1992, Analysis of Quantal Response Data: Chapman and Hall, London, England, 511 p.

Rosenbauer, R.J., Campbell, P.L., Lam, A., Lorenson, T.D., Hosstettler, F.D., Thomas, B., and Wong, F.L., 2011, Petroleum hydrocarbons in sediment from the northern Gulf of Mexico shoreline, Texas to Florida: U.S. Geolgocial Survey Open-File Report 2011-1014, 22 p.

SAS Institute, Inc., 1989, SAS/STAT ${ }^{\circledR}$ User's Guide, Version 6, 4th ed., Cary, North Carolina, v. 2, 846 p.

Thermo Fisher Scientific, Inc., 2007, User Guide, Silver/ Sulfide Ion Selective Electrode: Thermo Scientific, Inc., Beverly, Massachussets, 58 p.

United States Environmental Protection Agency, 1996, Marine Toxicity Identification Evaluation (TIE), Phase I Guidance Document: EPA/600/R-96/054, National Health and Environmental Effects Research Laboratory, Atlantic Ecology Division, Narragansett, Rhode Island, $54 \mathrm{p}$.

U.S. Geological Survey, 2007, Toxicity testing of sediments collected from coastal Louisiana, Mississippi, and Alabama following hurricane Katrina in 2005: Report prepared in collaboration with National Oceanic and Atmospheric Administration (NOAA) Center for Coastal Monitoring \& Assessment, NOAA National Centers for Coastal Ocean Science, and U.S. Environmental Protection Agency Gulf Ecology division, ORD. 8 p. +7 tables, 3 figures, and 4 attachments.

Winger, P.V., Albrecht, B., Anderson, B.S., Bay, S.M., Bona, F., Stephenson, G.L., 2003, Comparison of porewater and solid-phase sediment toxicity test: in Carr, R.S., and Nipper, M.G., eds., Porewater Toxicity Testing-Biological, Chemical and Ecological Considerations: Pensacola, Florida, USA: Society of Environmental Toxicology and Chemistry (SETAC), p. 37-61. 


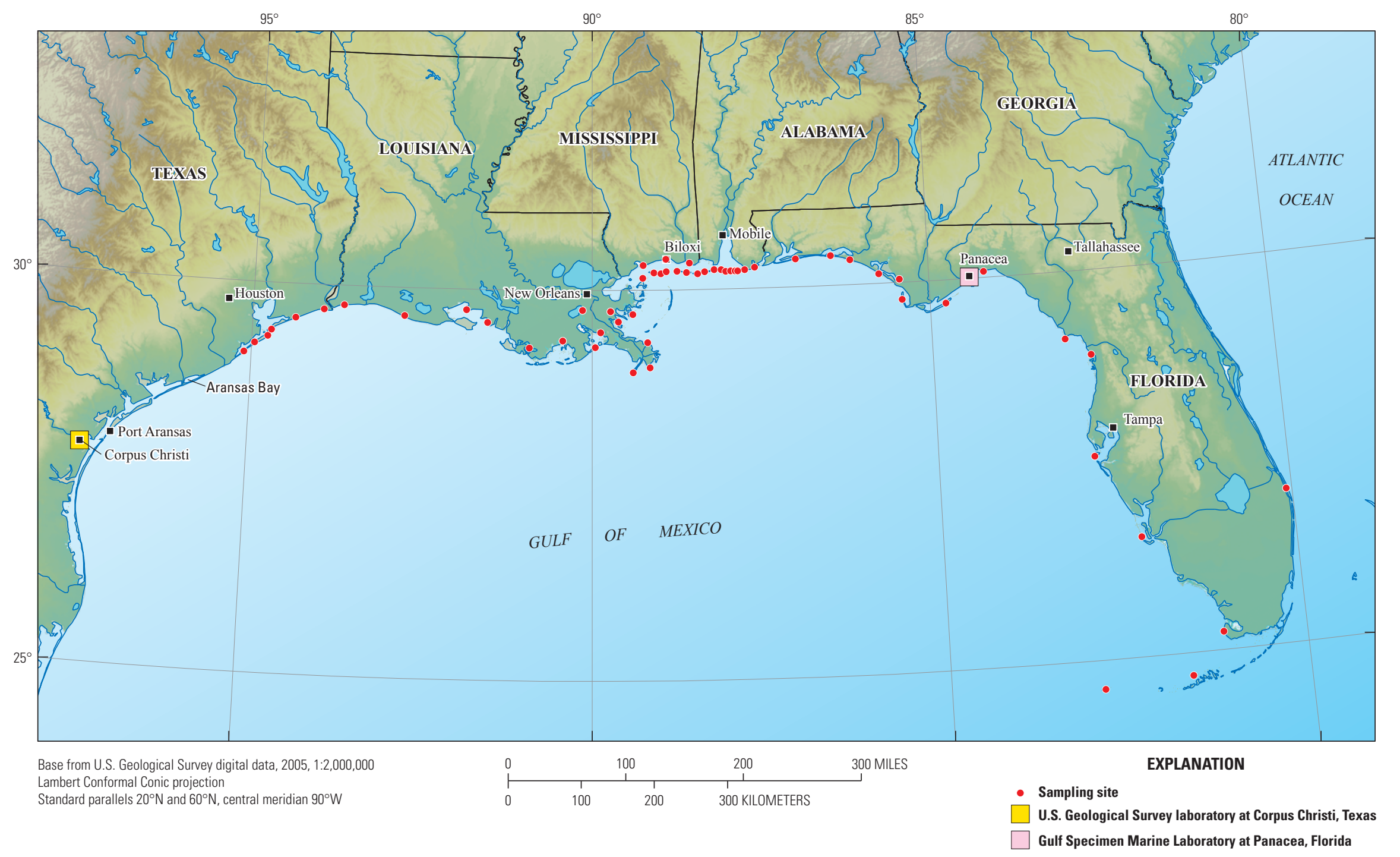

Figure 1. Sample sites in Texas, Louisiana, Mississppi, Alabama and Florida and locations of laboratories. 


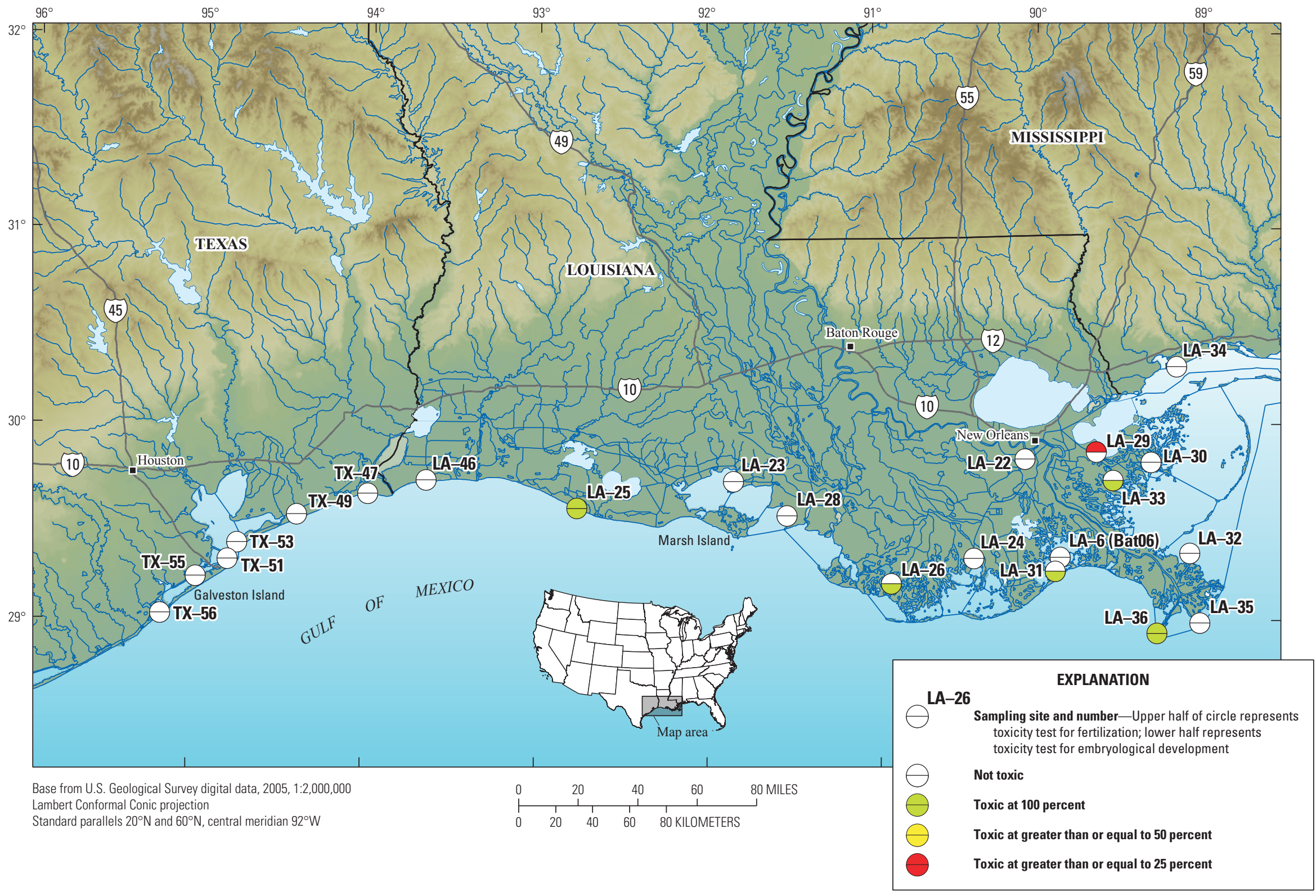

Figure 2. Sample sites in Texas and Louisiana and toxicity test results of the sea urchin fertilization and embryological development post-landfall tests of 100-, 50- and 25-percent salinity adjusted pore water. Color differentiation of circles indicates those sites that met the detectable significance criteria compared to the reference pore water. 


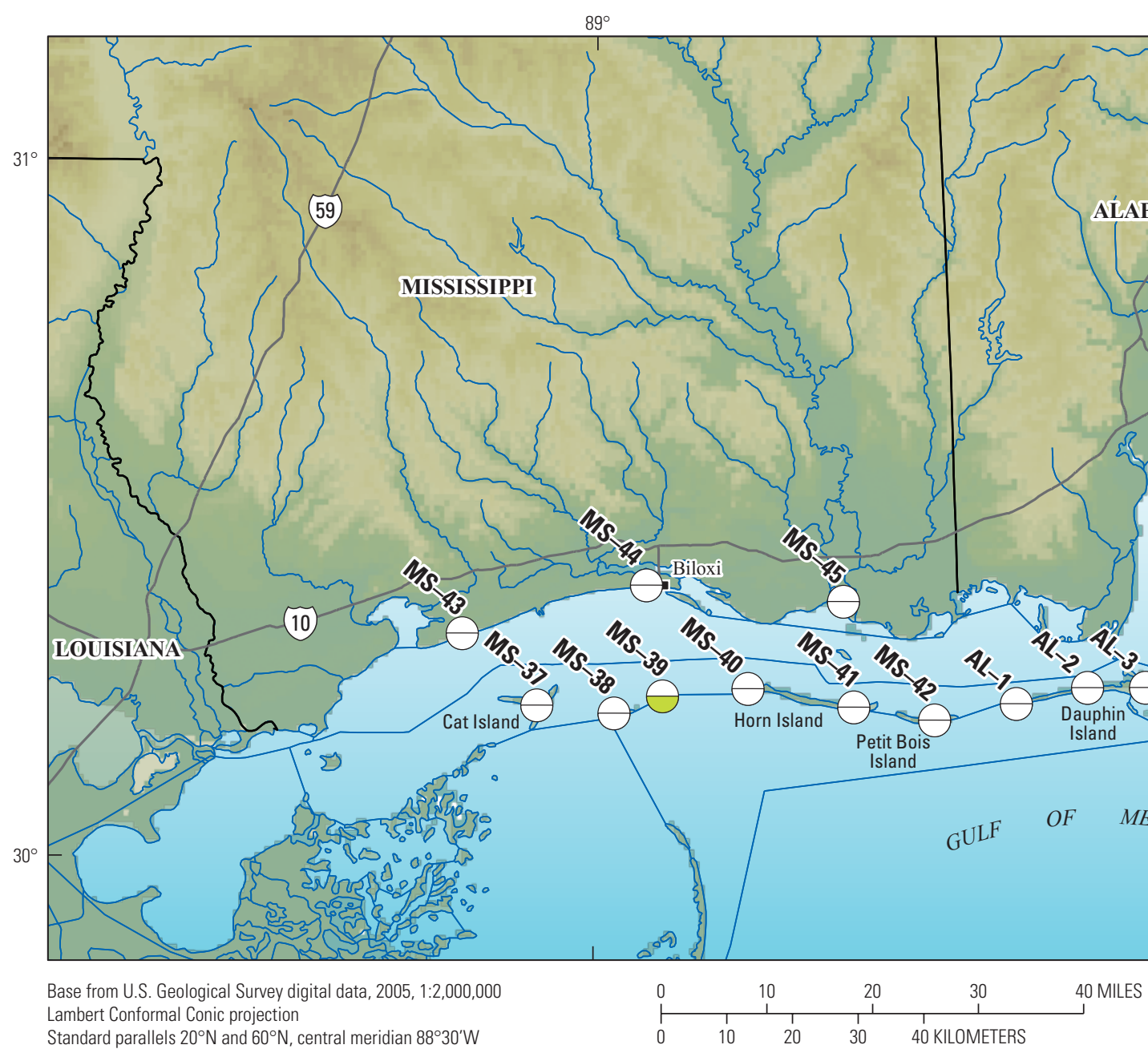

Figure 3. Sample sites in Mississippi and Alabama and toxicity test results of the sea urchin fertilization and embryological development post-landfall tests of 100-, 50- and 25-percent salinity adjusted pore water Color differentiation of circles indicates those sites that met the detectable significance criteria compared to the reference pore water. $88^{\circ}$ toxicity test for fertilization; lower half represents toxicity test for embryological development

\section{$\ominus \quad$ Not toxic}

$\bigoplus \quad$ Toxic at 100 percent

$\ominus \quad$ Toxic at greater than or equal to 50 percent

Toxic at greater than or equal to 25 percent 


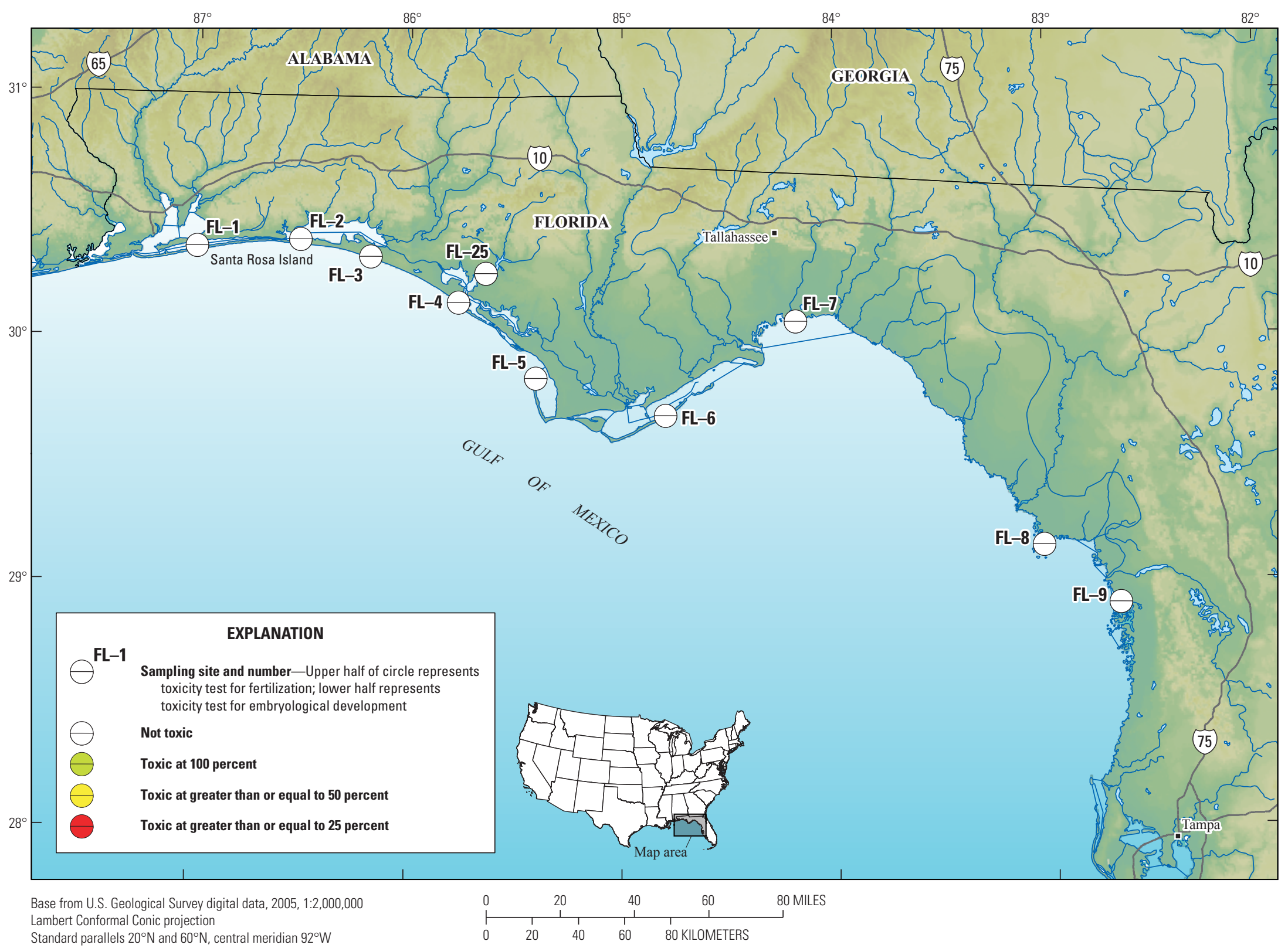

Figure 4. Sample sites in Florida and toxicity test results of the sea urchin fertilization and embryological development post-landfall tests of 100-, 50- and 25-percent salinity adjusted pore water. Color differentiation of circles indicates those sites that met the detectable significance criteria compared to the reference pore water. 


\section{Sediment Pore-Water Toxicity Test Results, Deepwater Horizon Oil Release, Gulf of Mexico, 2010}

Table 1. Relevant sample and extraction dates, times, temperatures and volumes of pore water collected from sediments pre-landfall of the Deepwater Horizon oil release.

[Site, U.S. Geological Survey (USGS) identifier for site locality; Site ID, USGS identifier number; Collection date and time, date and time sample was taken from the field; COC, chain of custody; temps., temperatures, in degrees Celsius; Extraction date, date pore water was collected at Marine Ecotoxicology Research Station Laboratory (MERS); (Rep 2), field replicate two; na, not available; (Rep 1), field replicate one]

\begin{tabular}{lcccccccc}
\hline \multicolumn{1}{c}{ Site } & Site ID & $\begin{array}{c}\text { Collection } \\
\text { date }\end{array}$ & $\begin{array}{c}\text { Collection } \\
\text { time }\end{array}$ & $\begin{array}{c}\text { Arrival } \\
\text { date }^{1}\end{array}$ & $\begin{array}{c}\text { Arrival COC } \\
\text { log-in time }^{2}\end{array}$ & $\begin{array}{c}\text { Incoming } \\
\text { sample temps. }^{3}\end{array}$ & $\begin{array}{c}\text { Extraction } \\
\text { date }^{2}\end{array}$ & $\begin{array}{c}\text { Volume } \\
\text { extracted }^{4}\end{array}$ \\
\hline FL-8 & 290740083031200 & $5 / 18 / 10$ & $17: 00$ & $5 / 21 / 2010$ & $11: 21$ & 7 & $5 / 21 / 2010$ & 110 \\
FL-9 & 285425082412600 & $5 / 19 / 10$ & $12: 00$ & $5 / 21 / 2010$ & $11: 21$ & $7^{5}$ & $5 / 21 / 2010$ & 170 \\
FL-11 (Rep 2) & 263132082114000 & $5 / 20 / 10$ & $16: 30$ & $5 / 26 / 2010$ & $11: 15$ & $8^{5}$ & $5 / 26 / 2010$ & 40 \\
FL-13 & 251329081101100 & $5 / 22 / 10$ & na & $5 / 26 / 2010$ & $11: 15$ & $8^{5}$ & $5 / 26 / 2010$ & 190 \\
FL-14 & 243737082522500 & $5 / 20 / 10$ & na & $5 / 26 / 2010$ & $11: 15$ & 8 & $5 / 26 / 2010$ & 180 \\
FL-19 & 265722080045400 & $6 / 16 / 10$ & $13: 00$ & $6 / 18 / 2010$ & $11: 05$ & 3 & $6 / 21 / 2010$ & 230 \\
FL-20 & 265722080045500 & $6 / 16 / 10$ & $15: 00$ & $6 / 18 / 2010$ & $11: 05$ & 3 & $6 / 21 / 2010$ & 180 \\
FL-24 & 273605082454900 & $6 / 14 / 10$ & $14: 00$ & $6 / 17 / 2010$ & $11: 00$ & 7.5 & $6 / 17 / 2010$ & 190 \\
FL-25 & 300223085260800 & $6 / 10 / 10$ & $11: 00$ & $6 / 17 / 2010$ & $15: 30$ & $7.5^{5}$ & $6 / 17 / 2010$ & 140 \\
FL-26 & 244325081351500 & $7 / 7 / 10$ & $11: 00$ & $7 / 9 / 2010$ & $15: 30$ & 3 & $7 / 12 / 2010$ & 140 \\
& & & & & & & & \\
LA-22 & 294432090083100 & $5 / 14 / 10$ & $12: 32$ & $5 / 21 / 2010$ & $11: 20$ & $6^{5}$ & $5 / 21 / 2010$ & 100 \\
LA-23 & 294406091511300 & $5 / 13 / 10$ & $12: 30$ & $5 / 21 / 2010$ & $11: 20$ & 6 & $5 / 21 / 2010$ & 170 \\
LA-23 (Rep 1) & 294406091511300 & $5 / 13 / 10$ & $12: 31$ & $5 / 21 / 2010$ & $11: 20$ & $6^{5}$ & $5 / 21 / 2010$ & 170 \\
LA-23 (Rep 2) & 294406091511300 & $5 / 13 / 10$ & $12: 32$ & $5 / 21 / 2010$ & $11: 20$ & 6 & $5 / 21 / 2010$ & 170 \\
LA-24 & 292046090254500 & $5 / 18 / 10$ & $16: 20$ & $5 / 21 / 2010$ & $11: 21$ & $7^{5}$ & $5 / 21 / 2010$ & 130 \\
LA-28 & 293424091321600 & $5 / 13 / 10$ & $9: 30$ & $5 / 21 / 2010$ & $11: 20$ & $6^{5}$ & $5 / 21 / 2010$ & 170 \\
LA-29 & 294324089432500 & $5 / 18 / 10$ & $14: 00$ & $5 / 21 / 2010$ & $11: 20$ & $6^{5}$ & $5 / 21 / 2010$ & 170 \\
\hline
\end{tabular}

${ }^{1}$ Date sample arrived by FedEx ${ }^{\circledR}$ into the USGS Marine Ecotoxicology Research Station Laboratory.

${ }^{2}$ Time chain of custody form was signed at the USGS Marine Ecotoxicology Research Station Laboratory.

${ }^{3}$ Incoming sample temperatures for individual jar of sediment for that site. Temperatures in degrees Celsius.

${ }^{4}$ Volume of pore water extracted in milliliters from the sample and stored frozen.

${ }^{5}$ Temperature estimate based on temperature taken of another sample in the same box. 
Table 2. Relevant sample and extraction dates, times, temperatures and volumes of pore water collected from sediments post-landfall of the Deepwater Horizon oil release.

[Site, U.S. Geological Survey (USGS) identifier for site locality; Site ID, USGS identifier number; Collection date and time: date and time sample was taken from the field; COC, chain of custody; temps., temperatures in degrees Celsius; Extraction date: date pore water was collected at Marine Ecotoxicology Research Station Laboratory (MERS)]

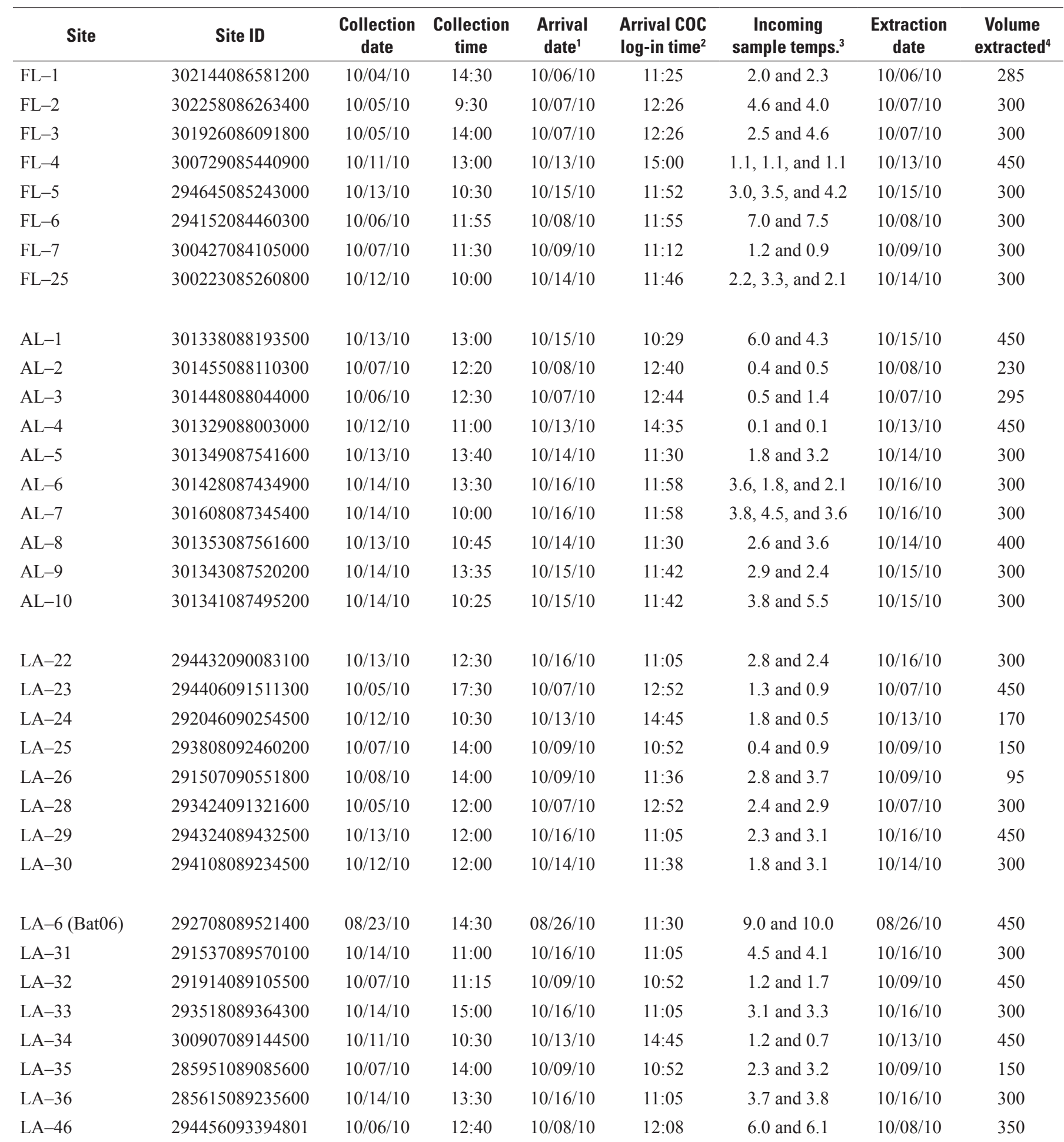




\section{Sediment Pore-Water Toxicity Test Results, Deepwater Horizon Oil Release, Gulf of Mexico, 2010}

Table 2. Relevant sample and extraction dates, times, temperatures and volumes of pore water collected from sediments post-landfall of the Deepwater Horizon oil release. - Continued

[Site, U.S. Geological Survey (USGS) identifier for site locality; Site ID, USGS identifier number; Collection date and time: date and time sample was taken from the field; COC, chain of custody; temps., temperatures in degrees Celsius; Extraction date: date pore water was collected at Marine Ecotoxicology Research Station Laboratory (MERS)]

\begin{tabular}{|c|c|c|c|c|c|c|c|c|}
\hline Site & Site ID & $\begin{array}{c}\text { Collection } \\
\text { date }\end{array}$ & $\begin{array}{c}\text { Collection } \\
\text { time }\end{array}$ & $\begin{array}{c}\text { Arrival } \\
\text { date }^{1}\end{array}$ & $\begin{array}{l}\text { Arrival COC } \\
\text { log-in time }\end{array}$ & $\begin{array}{c}\text { Incoming } \\
\text { sample temps. }^{3}\end{array}$ & $\begin{array}{c}\text { Extraction } \\
\text { date }\end{array}$ & $\begin{array}{c}\text { Volume } \\
\text { extracted }^{4}\end{array}$ \\
\hline MS-37 & 301309089044700 & $10 / 14 / 10$ & $11: 30$ & $10 / 16 / 10$ & $12: 30$ & 6.6 and 5.7 & $10 / 16 / 10$ & 300 \\
\hline MS-39 & 301358088533300 & $10 / 11 / 10$ & $14: 30$ & $10 / 13 / 10$ & $15: 08$ & 2.6 and 2.4 & $10 / 13 / 10$ & 400 \\
\hline MS-40 & 301425088440600 & $10 / 12 / 10$ & $16: 30$ & $10 / 14 / 10$ & $11: 56$ & 2.3 and 1.1 & $10 / 14 / 10$ & 450 \\
\hline MS-43 & 301858089141000 & $10 / 08 / 10$ & $10: 00$ & $10 / 09 / 10$ & $11: 28$ & 1.6 and 1.8 & $10 / 09 / 10$ & 400 \\
\hline MS-44 & 302336088535800 & $10 / 07 / 10$ & $10: 30$ & $10 / 09 / 10$ & $11: 18$ & 1.3 and 1.6 & $10 / 09 / 10$ & 400 \\
\hline MS-45 & 302034088325200 & $10 / 14 / 10$ & $14: 30$ & $10 / 16 / 10$ & $12: 30$ & 5.9 and 4.5 & $10 / 16 / 10$ & 450 \\
\hline TX-53 & 292318094430901 & $10 / 07 / 10$ & $12: 43$ & $10 / 09 / 10$ & $10: 38$ & 6.5 and 7.1 & $10 / 09 / 10$ & 150 \\
\hline TX-55 & 291251094571401 & $10 / 14 / 10$ & $11: 10$ & $10 / 16 / 10$ & $12: 15$ & 5.2 and 5.7 & $10 / 16 / 10$ & 300 \\
\hline $\mathrm{TX}-56$ & 290512095063101 & $10 / 05 / 10$ & $12: 50$ & $10 / 07 / 10$ & $12: 35$ & 4.2 and 5.6 & $10 / 07 / 10$ & 150 \\
\hline
\end{tabular}

${ }^{1}$ Date sample arrived by FedEx ${ }^{\circledR}$ into the USGS Marine Ecotoxicology Research Station Laboratory.

${ }^{2}$ Time chain of custody form was signed at the USGS Marine Ecotoxicology Research Station Laboratory.

${ }^{3}$ Incoming sample temperatures for 2 to 4 individual jars of sediment for that site. Temperatures in degrees Celsius.

${ }^{4}$ Volume of pore water extracted from the sample and stored frozen.

${ }^{5}$ Sample was recollected besause of insufficient pore-water extraction from the initial sample. 
Table 3. Water-quality measurements after salinity adjustment and original salinity of pore-water samples collected from sediments pre-landfall of the Deepwater Horizon oil release.

[Site, U.S. Geological Survey (USGS) identifier for site locality; Site ID, USGS identifier number; \%, parts per thousand; \%, percent; DO, dissolved oxygen; $\mathrm{mg} / \mathrm{L}$, milligrams per liter; TAN, total ammonia as nitrogen; UAN, un-ionized ammonia as nitrogen; $\mu \mathrm{g} / \mathrm{L}$, micrograms per liter; OS, original sample; <, less than; (Rep 2), field replicate two; (Rep 1), field replicate one; MFS, Millipore ${ }^{\circledR}$ filtered seawater; \pm , plus or minus]

\begin{tabular}{|c|c|c|c|c|c|c|c|c|c|}
\hline Site & Site ID & $\begin{array}{c}\text { Salinity }^{1} \\
\% \%\end{array}$ & $\begin{array}{c}\mathrm{DO} \\
(\mathrm{mg} / \mathrm{L})\end{array}$ & \% DO & $\mathrm{pH}$ & $\begin{array}{c}\text { TAN } \\
\text { (mg/L) }\end{array}$ & $\begin{array}{c}\text { UAN } \\
(\mu \mathrm{g} / \mathrm{L})\end{array}$ & $\begin{array}{c}\text { Sulfide } \\
\text { (mg/L) }\end{array}$ & $\% \mathbf{O S}^{2}$ \\
\hline FL-8 & 290740083031200 & 30 & 7.24 & 99.1 & 8.10 & 0.667 & 26.2 & $<0.009$ & 100 \\
\hline FL-11 (Rep 2) & 263132082114000 & 22.5 & 7.44 & 100.6 & 8.06 & 5.97 & 214.8 & .009 & 90.9 \\
\hline FL-13 & 251329081101100 & 20 & 7.31 & 98.9 & 8.05 & 1.54 & 54.4 & $<.009$ & 88.0 \\
\hline FL-20 & 265722080045500 & 33 & 7.08 & 95.2 & 7.97 & .178 & 5.3 & $<.009$ & 90.9 \\
\hline FL-24 & 273605082454900 & 35.5 & 7.30 & 97.8 & 7.91 & .809 & 20.5 & $<.009$ & 84.5 \\
\hline FL-25 & 300223085260800 & 14 & 7.47 & 100.8 & 8.17 & $<.1$ & $<4.6$ & $<.009$ & 81.9 \\
\hline FL-26 & 244325081351500 & 40 & 7.44 & 100.1 & 8.06 & 1.32 & 46.8 & $<.009$ & 75.3 \\
\hline LA-23 (Rep 1) & 294406091511300 & 4 & 7.24 & 97.6 & 8.25 & $<.1$ & $<5.5$ & $<.009$ & 73.6 \\
\hline LA-23 (Rep 2) & 294406091511300 & 4 & 7.10 & 95.9 & 8.26 & $<.1$ & $<5.5$ & $<.009$ & 73.6 \\
\hline LA-24 & 292046090254500 & 22 & 7.51 & 101.5 & 8.14 & .171 & 7.3 & $<.009$ & 90.3 \\
\hline LA-28 & 293424091321600 & 0.5 & 7.36 & 99.4 & 8.31 & $<.1$ & $<6.3$ & $<.009$ & 71.1 \\
\hline LA-29 & 294324089432500 & 2 & 7.07 & 95.7 & 8.21 & .32 & 16.0 & $<.009$ & 72.0 \\
\hline $\mathrm{TXREF}^{3}$ & None & 22.5 & 7.52 & 101.8 & 8.31 & .184 & 11.3 & $<.009$ & 90.5 \\
\hline $\mathrm{MFS}^{4}$ & None & 32.5 & 6.34 & 86.5 & 8.12 & $<.1$ & $<4.1$ & $<.009$ & 92.3 \\
\hline Brine blank ${ }^{5}$ & None & 6 & 6.14 & 83.3 & 8.35 & $<.1$ & $<6.8$ & $<.009$ & 20.5 \\
\hline
\end{tabular}

\footnotetext{
${ }^{1}$ Salinty of sample before adjustment. Sample adjusted to $30 \pm 1 \%$.

${ }^{2}$ Percent of original sample after salinity adjustment.

${ }^{3}$ Reference pore water extracted from sediment collected in Aransas Bay, Texas.

${ }^{4}$ Millipore ${ }^{\circledR}$ filtered seawater diluent.

${ }^{5}$ Brine blank of TXREF diluted to $6 \%$ with Milli-Q ${ }^{\circledR}$ purified water and subsequently increased with brine to $30 \%$ (concentrated brine at $102 \%$ ).
} 
Table 4. Sea urchin fertilization test raw data and means for pore-water samples collected from sediments pre-landfall of the Deepwater Horizon oil release.

[Site, U.S. Geological Survey (USGS) identifier for site locality; Site ID, USGS identifier number; \%, percent; WQAS, water quality adjusted sample; SD, standard deviation; na, not available; (Rep 2), field replicate two; (Rep 1), field replicate one; MFS, Millipore ${ }^{\mathbb{B}}$ filtered seawater; SDS, sodium dodecyl sulfate]

\begin{tabular}{|c|c|c|c|c|c|c|c|c|c|c|}
\hline \multirow{2}{*}{ Site } & \multirow{2}{*}{ Site ID } & \multirow{2}{*}{$\begin{array}{c}\% \\
\text { WOAS }^{1}\end{array}$} & \multicolumn{5}{|c|}{$\%$ Fertilized } & \multirow{2}{*}{ Mean } & \multirow{2}{*}{ SD } & \multirow{2}{*}{$\begin{array}{c}\% \text { of } \\
\text { control }^{2}\end{array}$} \\
\hline & & & $\operatorname{Rep} 1$ & $\operatorname{Rep} 2$ & Rep 3 & Rep 4 & $\operatorname{Rep} 5$ & & & \\
\hline \multirow[t]{2}{*}{$\mathrm{FL}-8$} & 290740083031200 & 100 & 100 & 100 & 100 & 99 & 100 & 99.8 & 0.4 & 101.1 \\
\hline & & 25 & 98 & 100 & 100 & 98 & na & 99.0 & 1.2 & 99.9 \\
\hline \multirow[t]{2}{*}{ FL-9 } & 285425082412600 & 100 & 100 & 99 & 100 & 100 & 100 & 99.8 & .4 & 101.1 \\
\hline & & 25 & 99 & 100 & 100 & 99 & 100 & 99.6 & .5 & 100.5 \\
\hline FL-11 (Rep 2) & 263132082114000 & 100 & 91 & 95 & 94 & 93 & na & 93.3 & 1.7 & 94.5 \\
\hline \multirow[t]{3}{*}{ FL-13 } & 251329081101100 & 100 & 98 & 100 & 100 & 99 & 100 & 99.4 & .9 & 100.7 \\
\hline & & 50 & 99 & 100 & 99 & 100 & 100 & 99.6 & .5 & 100.5 \\
\hline & & 25 & 100 & 100 & 100 & 99 & 100 & 99.8 & .4 & 100.7 \\
\hline \multirow[t]{3}{*}{ FL-19 } & 265722080045400 & 100 & 100 & 100 & 100 & 100 & 99 & 99.8 & .4 & 101.1 \\
\hline & & 50 & 99 & 100 & 100 & 100 & 99 & 99.6 & .5 & 100.5 \\
\hline & & 25 & 99 & 100 & 100 & 100 & 100 & 99.8 & .4 & 100.7 \\
\hline \multirow[t]{3}{*}{ FL-20 } & 265722080045500 & 100 & 100 & 100 & 100 & 99 & 100 & 99.8 & .4 & 101.1 \\
\hline & & 50 & 99 & 98 & 99 & 100 & 99 & 99.0 & .7 & 99.9 \\
\hline & & 25 & 99 & 100 & 100 & 100 & 100 & 99.8 & .4 & 100.7 \\
\hline \multirow[t]{2}{*}{ FL-24 } & 273605082454900 & 100 & 99 & 100 & 99 & 98 & 99 & 99.0 & .7 & 100.3 \\
\hline & & 50 & 99 & 100 & 98 & 99 & 99 & 99.0 & .7 & 99.9 \\
\hline FL-26 & & 25 & 100 & 100 & 100 & 99 & 99 & 99.6 & .5 & 100.5 \\
\hline \multirow[t]{3}{*}{ LA-22 } & 294432090083100 & 100 & 100 & 100 & 98 & 99 & 99 & 99.2 & .8 & 100.5 \\
\hline & & 50 & 100 & 100 & 99 & 100 & 98 & 99.4 & .9 & 100.3 \\
\hline & & 25 & 100 & 99 & 99 & 100 & 100 & 99.6 & .5 & 100.5 \\
\hline \multirow[t]{3}{*}{$\mathrm{LA}-23$} & 294406091511300 & 100 & 99 & 99 & 100 & 99 & 99 & 99.2 & .4 & 100.5 \\
\hline & & 50 & 98 & 100 & 99 & 99 & 100 & 99.2 & .8 & 100.1 \\
\hline & & 25 & 100 & 99 & 100 & 98 & 100 & 99.4 & .9 & 100.3 \\
\hline \multirow[t]{3}{*}{ LA-23 (Rep 1) } & 294406091511300 & 100 & 99 & 98 & 100 & 100 & 100 & 99.4 & .9 & 100.7 \\
\hline & & 50 & 99 & 99 & 99 & 99 & 97 & 98.6 & .9 & 99.5 \\
\hline & & 25 & 100 & 100 & 98 & 98 & 99 & 99.0 & 1.0 & 99.9 \\
\hline \multirow[t]{3}{*}{ LA-23 (Rep 2) } & 294406091511300 & 100 & 100 & 100 & 100 & 98 & 100 & 99.6 & .9 & 100.9 \\
\hline & & 50 & 98 & 100 & 98 & 100 & 99 & 99.0 & 1.0 & 99.9 \\
\hline & & 25 & 99 & 100 & 98 & 99 & 99 & 99.0 & .7 & 99.9 \\
\hline
\end{tabular}


Table 4. Sea urchin fertilization test raw data and means for pore-water samples collected from sediments pre-landfall of the Deepwater Horizon oil release. —Continued

[Site, U.S. Geological Survey (USGS) identifier for site locality; Site ID, USGS identifier number; \%, percent; WQAS, water quality adjusted sample; SD, standard deviation; na, not available; (Rep 2), field replicate two; (Rep 1), field replicate one; MFS, Millipore ${ }^{\circledR}$ filtered seawater; SDS, sodium dodecyl sulfate]

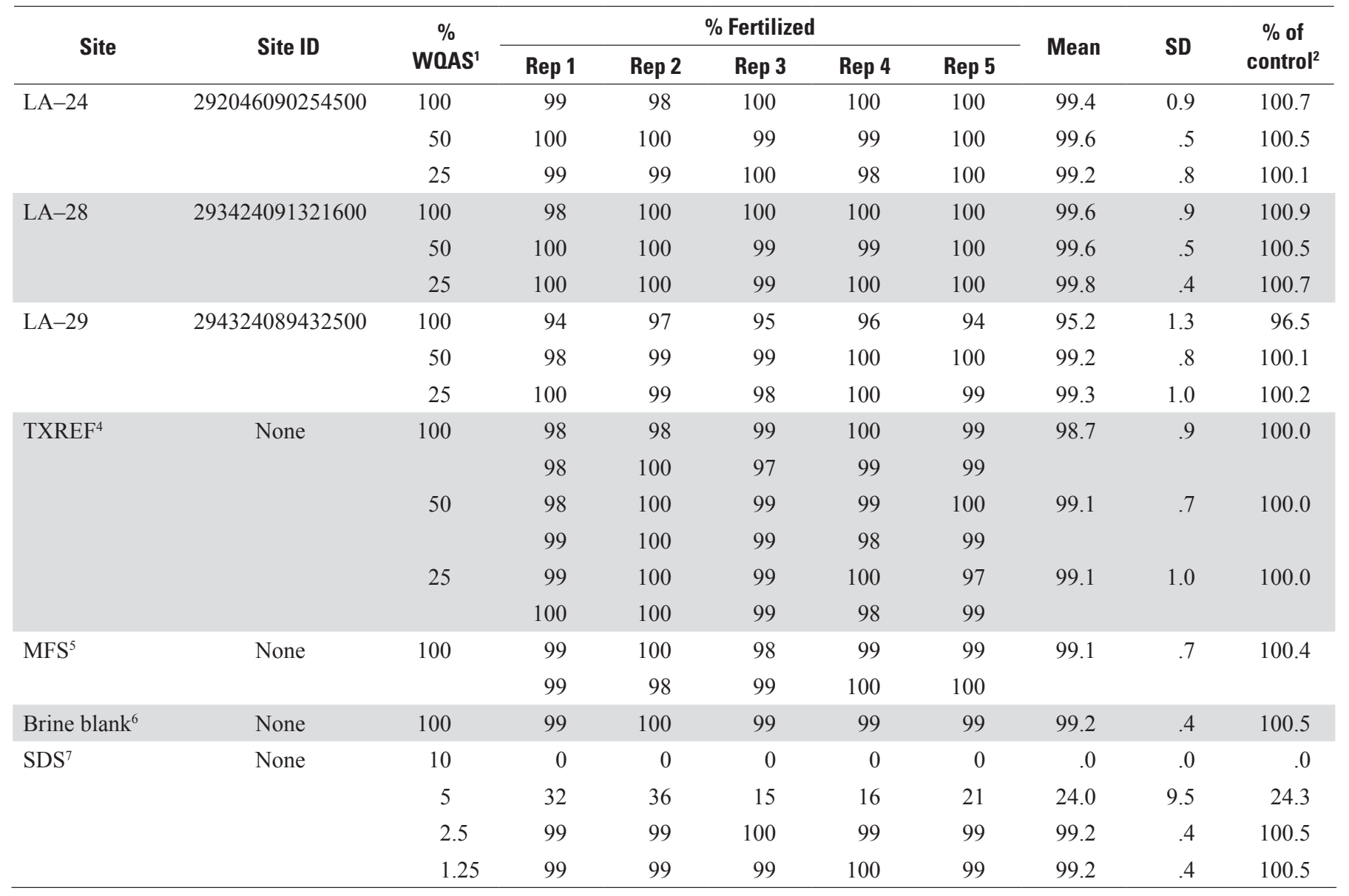

${ }^{1}$ Percent of salinity adjusted pore-water sample or concentration of SDS reference toxicant in milligrams per liter.

${ }^{2}$ Percent of TXREF control at the appropriate dilution.

${ }^{3}$ Data point not available because of error in pipetting.

${ }^{4}$ Reference pore water extracted from sediment collected in Aransas Bay, Texas.

${ }^{5}$ Millipore ${ }^{\circledR}$ filtered seawater diluent.

${ }^{6}$ Brine blank consisting of TXREF reference pore water diluted to $6 \%$ and subsequently increased with brine to $30 \%$ with concentrated brine.

${ }^{7}$ Sodium dodecyl sulfate positive control (in milligrams per liter). 
Table 5. Sea urchin embryological development test raw data and means for pore-water samples collected from sediments pre-landfall of the Deepwater Horizon oil release.

[Site, U.S. Geological Survey (USGS) identifier for site locality; Site ID, USGS identifier number; \%, percent; WQAS, water quality adjusted sample; Rep, replicate; SD, standard deviation; na, not available; (Rep 2), field replicate two; (Rep 1), field replicate one; MFS, Millipore ${ }^{\circledR}$ filtered seawater; SDS, sodium dodecyl sulfate]

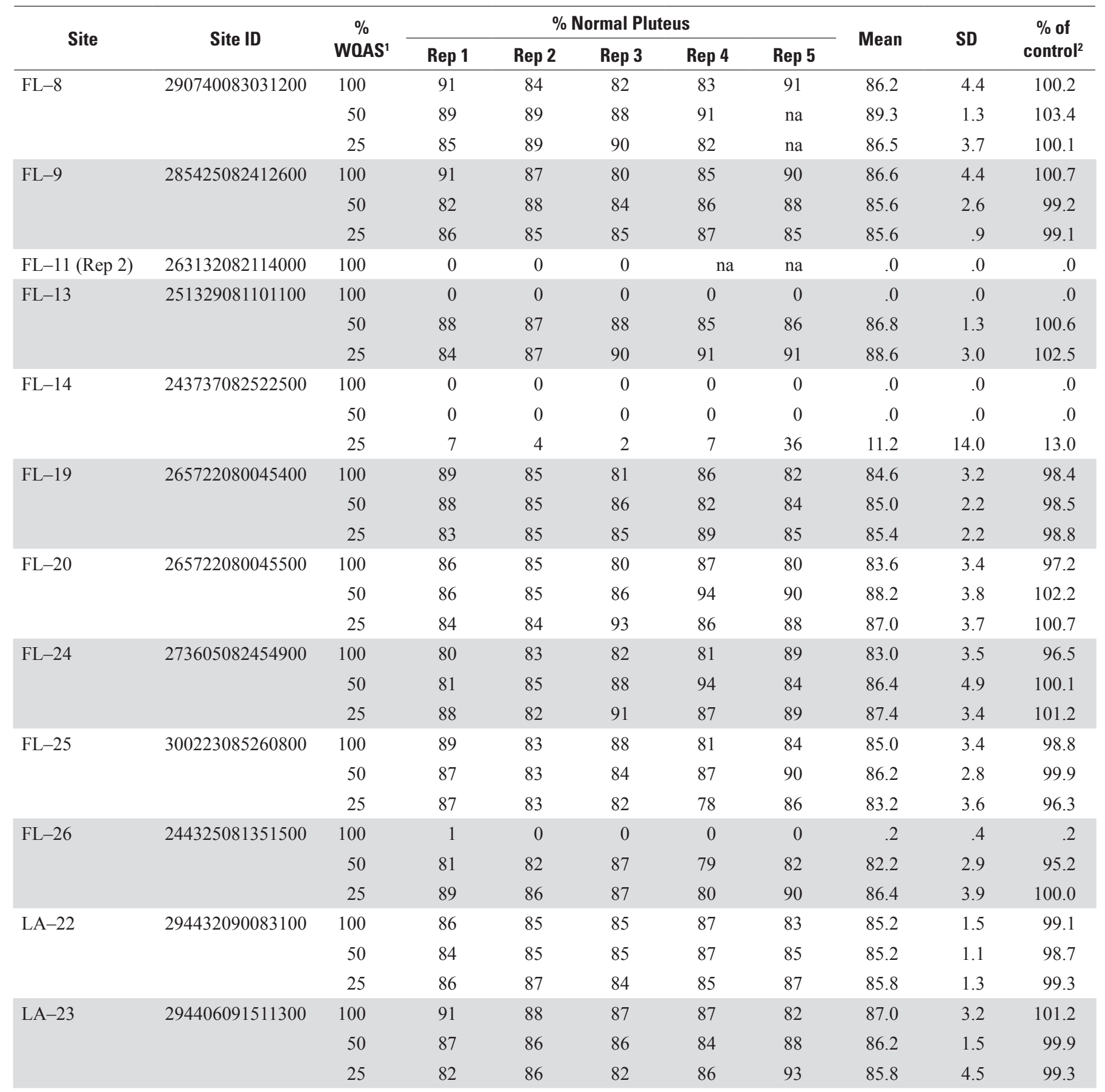


Table 5. Sea urchin embryological development test raw data and means for pore-water samples collected from sediments pre-landfall of the Deepwater Horizon oil release.-Continued

[Site, U.S. Geological Survey (USGS) identifier for site locality; Site ID, USGS identifier number; \%, percent; WQAS, water quality adjusted sample; Rep, replicate; SD, standard deviation; na, not available; (Rep 2), field replicate two; (Rep 1), field replicate one; MFS, Millipore ${ }^{\circledR}$ filtered seawater; SDS, sodium dodecyl sulfate]

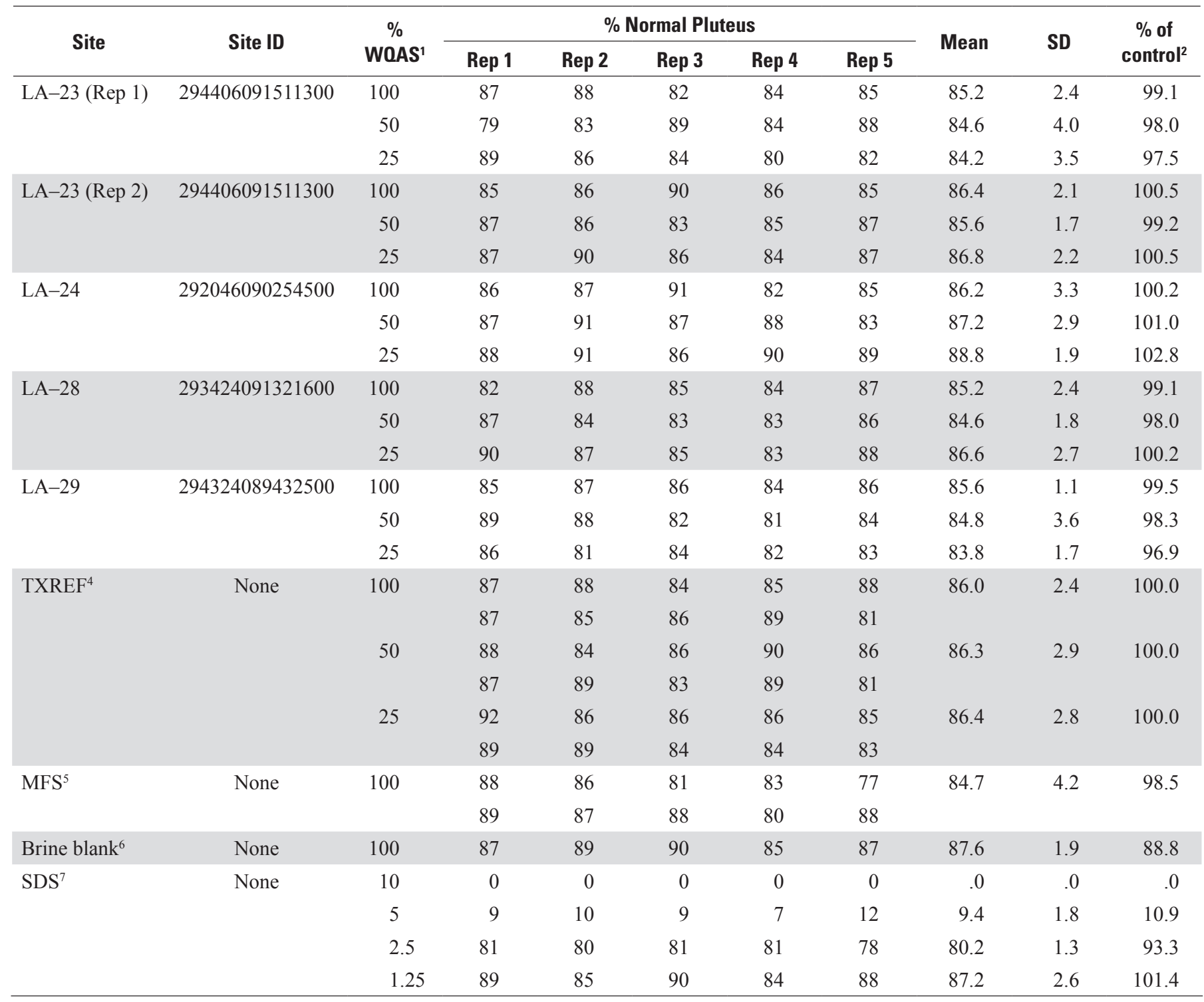

${ }^{1}$ Percent of salinity adjusted pore-water sample or concentration of SDS reference toxicant in milligrams per liter.

${ }^{2}$ Percent of TXREF control at the appropriate dilution.

${ }^{3}$ Data point not available because of error in pipetting.

${ }^{4}$ Reference pore water extracted from sediment collected in Aransas Bay, Texas.

${ }^{5}$ Millipore ${ }^{\circledR}$ filtered seawater diluent.

${ }^{6}$ Brine blank consisting of TXREF reference pore water diluted to $6 \%$ and subsequently increased with brine to $30 \%$ with concentrated brine.

${ }^{7}$ Sodium dodecyl sulfate positive control (in milligrams per liter). 
Table 6. Water-quality measurements after salinity adjustment and original salinity of pore-water samples collected from sediments post-landfall of the Deepwater Horizon oil release.

[Site, U.S. Geological Survey (USGS) identifier for site locality; Site ID, USGS identifier number; \%o, parts per thousand; \%, percent; DO, dissolved oxygen; $\mathrm{mg} / \mathrm{L}$, milligrams per liter; TAN, total ammonia as nitrogen; UAN, un-ionized ammonia as nitrogen; $\mu \mathrm{g} / \mathrm{L}$, micrograms per liter; OS, original sample; <, less than; (Rep 1), field replicate one; (Rep 2), field replicate two; MFS, Millipore ${ }^{\circledR}$ filtered seawater; \pm , plus or minus]

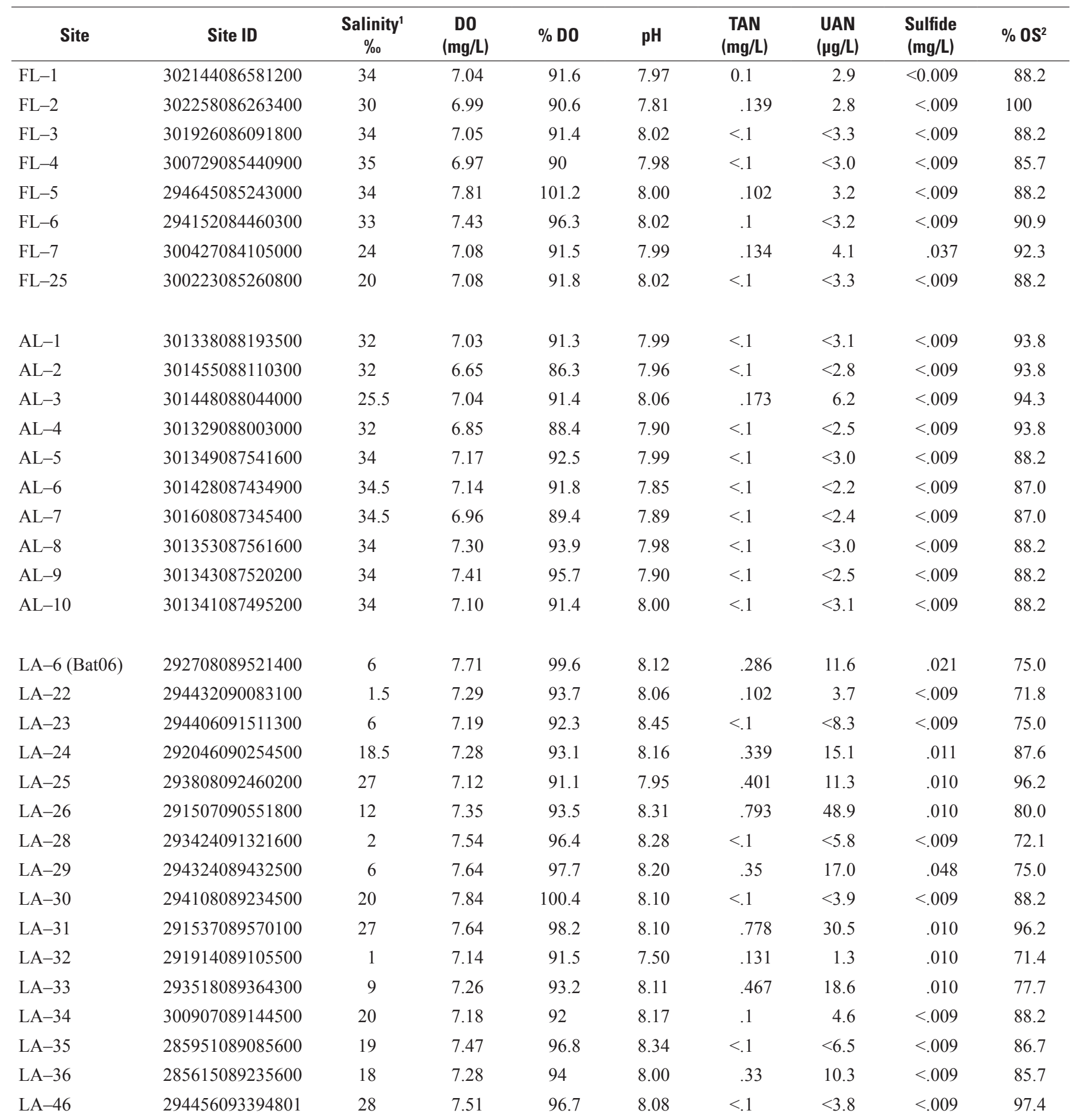


Table 6. Water-quality measurements after salinity adjustment and original salinity of pore-water samples collected from sediments post-landfall of the Deepwater Horizon oil release.-Continued

[Site, U.S. Geological Survey (USGS) identifier for site locality; Site ID, USGS identifier number; \%, parts per thousand; \%, percent; DO, dissolved oxygen; $\mathrm{mg} / \mathrm{L}$, milligrams per liter; TAN, total ammonia as nitrogen; UAN, un-ionized ammonia as nitrogen; $\mu \mathrm{g} / \mathrm{L}$, micrograms per liter; OS, original sample; <, less than; (Rep 1), field replicate one; (Rep 2), field replicate two; MFS, Millipore ${ }^{\circledR}$ filtered seawater; \pm , plus or minus]

\begin{tabular}{|c|c|c|c|c|c|c|c|c|c|}
\hline Site & Site ID & $\begin{array}{c}\text { Salinity }^{1} \\
\% 0\end{array}$ & $\begin{array}{c}\text { DO } \\
(\mathrm{mg} / \mathrm{L})\end{array}$ & \% DO & pH & $\begin{array}{c}\text { TAN } \\
\text { (mg/L) }\end{array}$ & $\begin{array}{c}\text { UAN } \\
(\mu \mathrm{g} / \mathrm{L})\end{array}$ & $\begin{array}{l}\text { Sulfide } \\
\text { (mg/L) }\end{array}$ & $\% \mathbf{O S}^{2}$ \\
\hline MS-37 & 301309089044700 & 29 & 7.24 & 93.2 & 7.84 & $<.1$ & $<2.2$ & $<0.009$ & 100.0 \\
\hline MS-39 & 301358088533300 & 29 & 7.34 & 94.1 & 7.96 & .237 & 6.7 & $<.009$ & 100.0 \\
\hline $\mathrm{MS}-40$ & 301425088440600 & 32 & 7.47 & 95.7 & 8.12 & $<.1$ & $<4.1$ & $<.009$ & 93.8 \\
\hline MS-43 & 301858089141000 & 19 & 7.54 & 96.7 & 8.02 & .171 & 5.6 & .017 & 86.7 \\
\hline MS-44 & 302336088535800 & 23 & 7.55 & 97 & 8.04 & $<.1$ & $<3.4$ & $<.009$ & 91.2 \\
\hline $\mathrm{MS}-45$ & 302034088325200 & 28 & 7.83 & 100.8 & 7.87 & $<.1$ & $<2.4$ & .010 & 94.7 \\
\hline $\mathrm{TX}-53$ & 292318094430901 & 26 & 7.68 & 98.9 & 8.32 & .108 & 6.9 & .009 & 94.9 \\
\hline $\mathrm{TX}-55$ & 291251094571401 & 27 & 7.44 & 96 & 7.98 & $<.1$ & $<3.0$ & .010 & 96.1 \\
\hline $\mathrm{TX}-56$ & 290512095063101 & 30 & 7.43 & 95.9 & 8.11 & .157 & 6.3 & $<.009$ & 100.0 \\
\hline TXREF $^{3}$ & None & 23 & 7.57 & 98.6 & 8.32 & .24 & 15.1 & .010 & 90.6 \\
\hline $\mathrm{MFS}^{4}$ & None & 30 & 6.49 & 84.3 & 8.13 & $<.1$ & $<4.1$ & $<.009$ & 100.0 \\
\hline Brine blank $^{5}$ & None & 23 & 6.22 & 80.9 & 8.47 & $<.1$ & $<8.6$ & $<.009$ & 18.8 \\
\hline Rinse blank ${ }^{6}$ & None & 30 & 7.08 & 92.2 & 8.18 & $<.1$ & $<4.7$ & $<.009$ & 100.0 \\
\hline
\end{tabular}

${ }^{1}$ Salinty of sample before adjustment. Sample adjusted to $30 \pm 1 \%$.

${ }^{2}$ Percent of original sample after salinity adjustment.

${ }^{3}$ Reference pore water extracted from sediment collected in Aransas Bay, Texas.

${ }^{4}$ Millipore ${ }^{\circledR}$ filtered seawater diluent.

${ }^{5}$ Brine blank of TXREF diluted to $6 \%$ with Milli-Q ${ }^{\circledR}$ purified water and subsequently increased with brine to $30 \%$ (concentrated brine at $102 \%$ ).

${ }^{6}$ Rinsate blank consisting of filtered seawater run over a complete set of all equipment used in pore-water extraction. 
Table 7. Sea urchin fertilization test raw data and means for experiment one with pore-water samples collected from sediments post-landfall of the Deepwater Horizon oil release.

[Site, U.S. Geological Survey (USGS) identifier for site locality; Site ID, USGS identifier number; \%, percent; WQAS, water quality adjustment sample; Rep, replicate; SD, standard deviation; na, not available; MFS, Millipore ${ }^{\circledR}$ filtered seawater; SDS, sodium dodecyl sulfate]

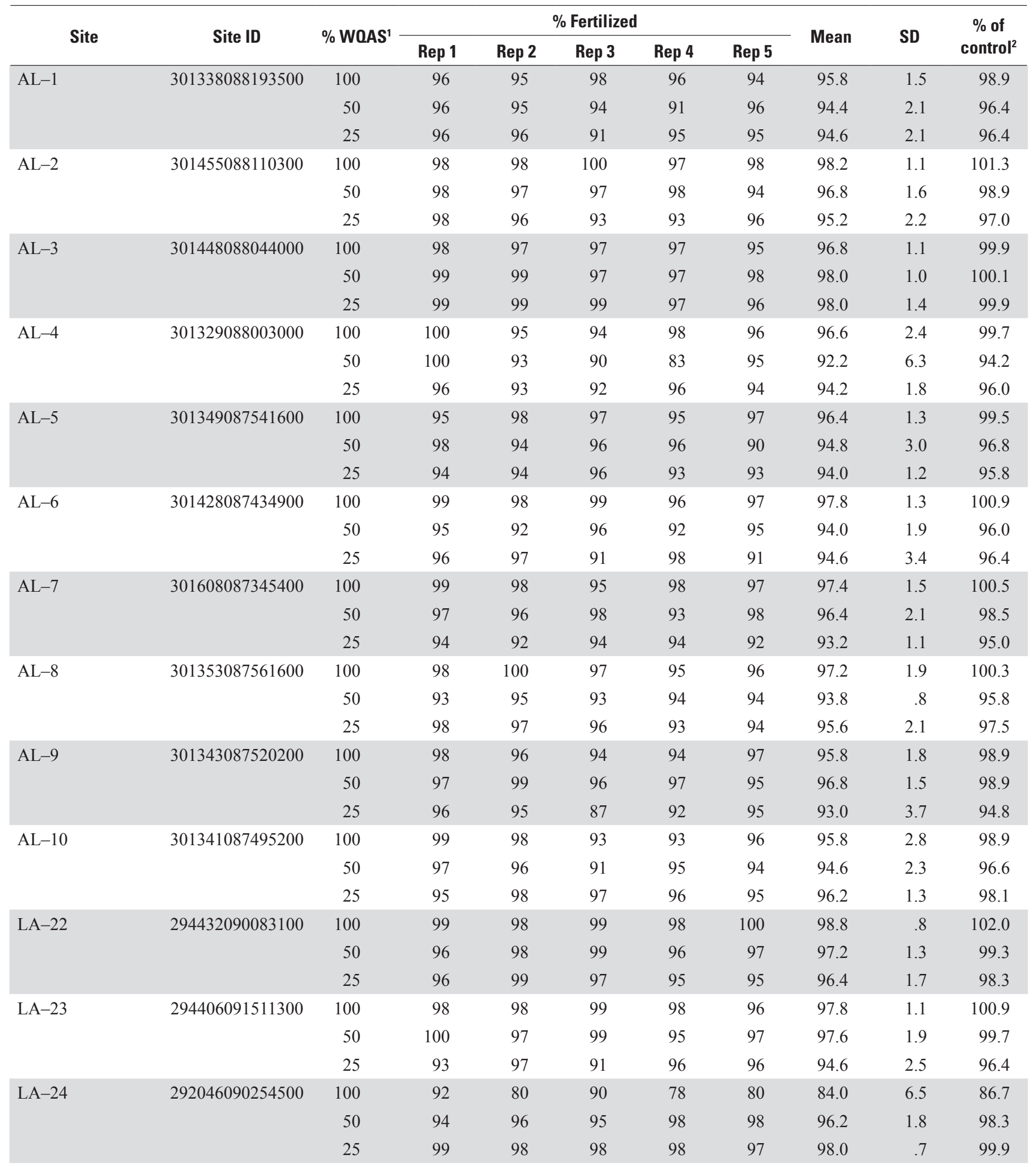


Table 7. Sea urchin fertilization test raw data and means for experiment one with pore-water samples collected from sediments post-landfall of the Deepwater Horizon oil release. - Continued

[Site, U.S. Geological Survey (USGS) identifier for site locality; Site ID, USGS identifier number; \%, percent; WQAS, water quality adjustment sample; Rep, replicate; SD, standard deviation; na, not available; MFS, Millipore ${ }^{\circledR}$ filtered seawater; SDS, sodium dodecyl sulfate]

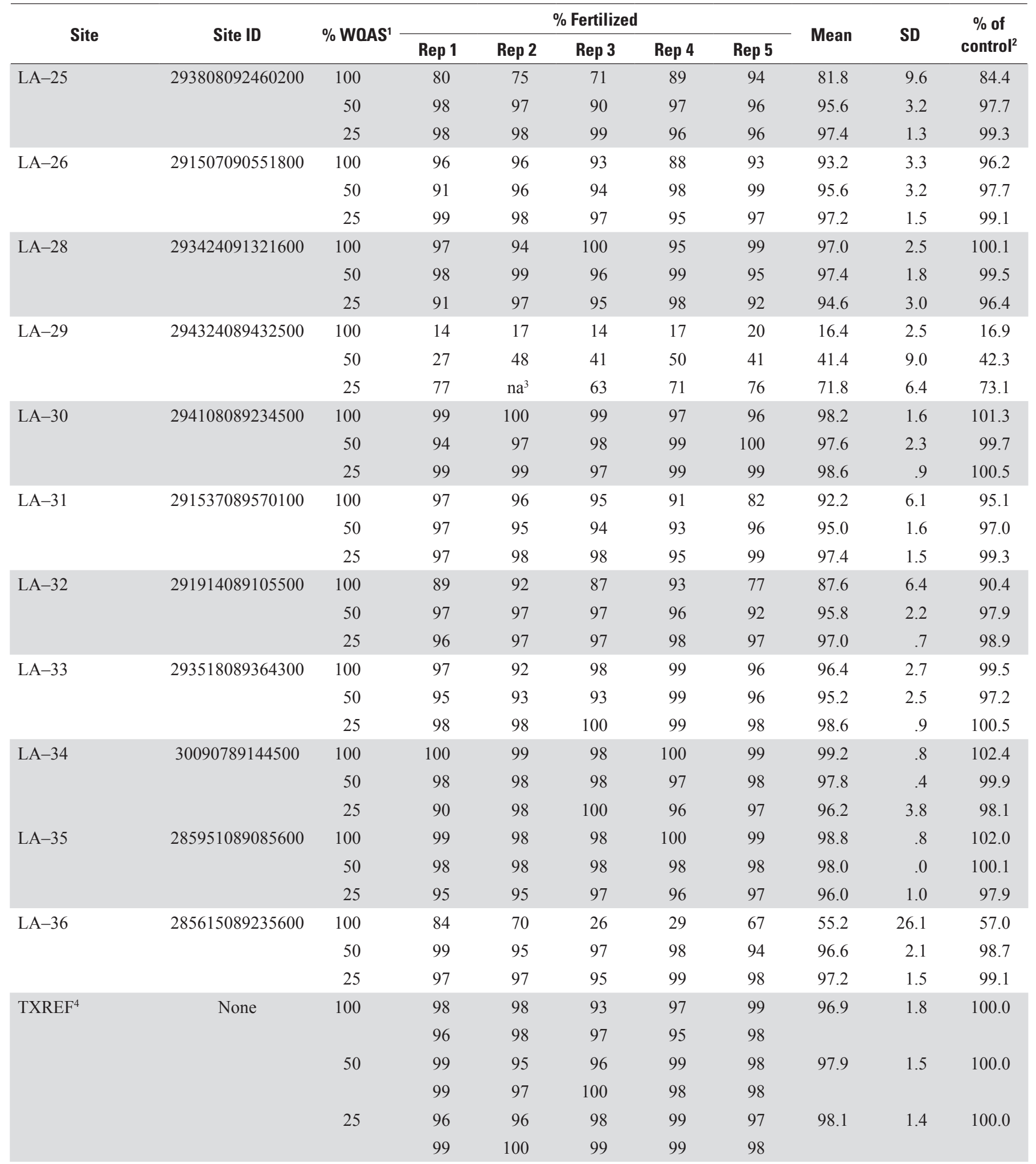


24 Sediment Pore-Water Toxicity Test Results, Deepwater Horizon Oil Release, Gulf of Mexico, 2010

Table 7. Sea urchin fertilization test raw data and means for experiment one with pore-water samples collected from sediments post-landfall of the Deepwater Horizon oil release. - Continued

[Site, U.S. Geological Survey (USGS) identifier for site locality; Site ID, USGS identifier number; \%, percent; WQAS, water quality adjustment sample; Rep, replicate; SD, standard deviation; na, not available; MFS, Millipore ${ }^{\circledR}$ filtered seawater; SDS, sodium dodecyl sulfate]

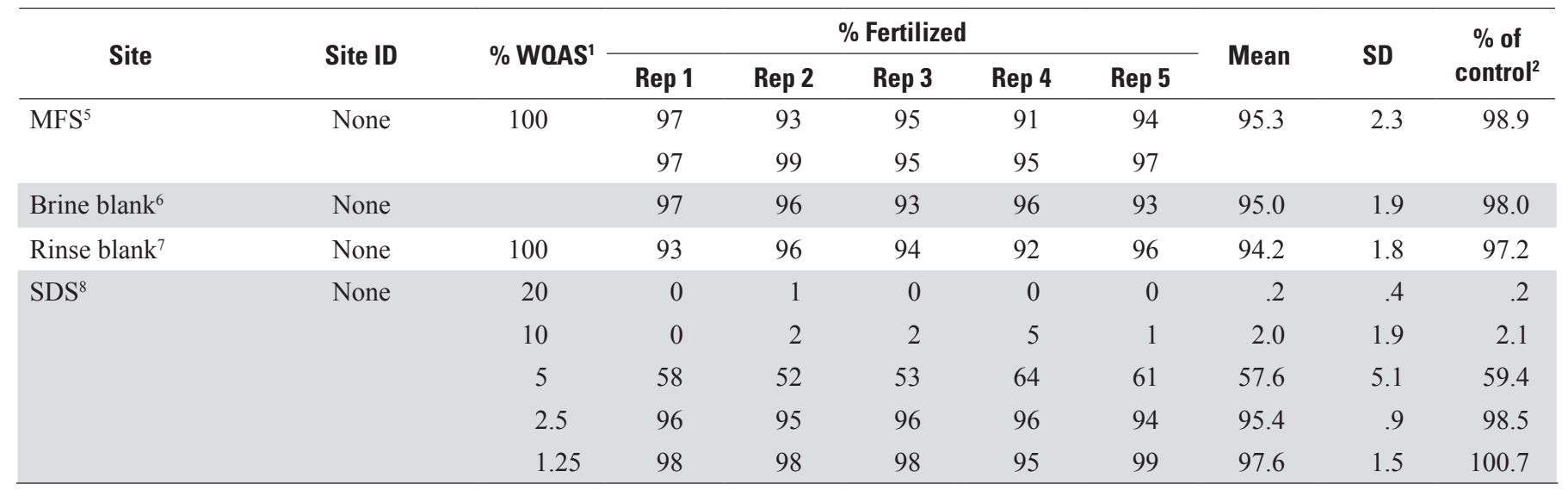

${ }^{1}$ Percent of salinity adjusted pore-water sample or concentration of SDS reference toxicant in milligrams per liter.

${ }^{2}$ Percent of TXREF control at the appropriate dilution.

${ }^{3}$ Data point not available because of error in pipetting.

${ }^{4}$ Reference pore water extracted from sediment collected in Aransas Bay, Texas.

${ }^{5}$ Millipore ${ }^{\circledR}$ filtered seawater diluent.

${ }^{6}$ Brine blank consisting of TXREF reference pore water diluted to $6 \%$ and subsequently increased with brine to $30 \%$ with concentrated brine.

${ }^{7}$ Rinsate blank consisting of filtered seawater run over a complete set of all equipment used in pore-water extraction.

${ }^{8}$ Sodium dodecyl sulfate positive control (in milligrams per liter). 
Table 8. Sea urchin fertilization test raw data and means for experiment two with pore-water samples collected from sediments post-landfall of the Deepwater Horizon oil release.

[Site, U.S. Geological Survey (USGS) identifier for site locality; Site ID, USGS identifier number; \%, percent; WQAS, water quality adjusted sample; Rep, replicate; SD, standard deviation; na, not available; (Rep 2), field replicate two; (Rep 1), field replicate one; MFS, Millipore ${ }^{\circledR}$ filtered seawater; SDS, sodium dodecyl sulfate]

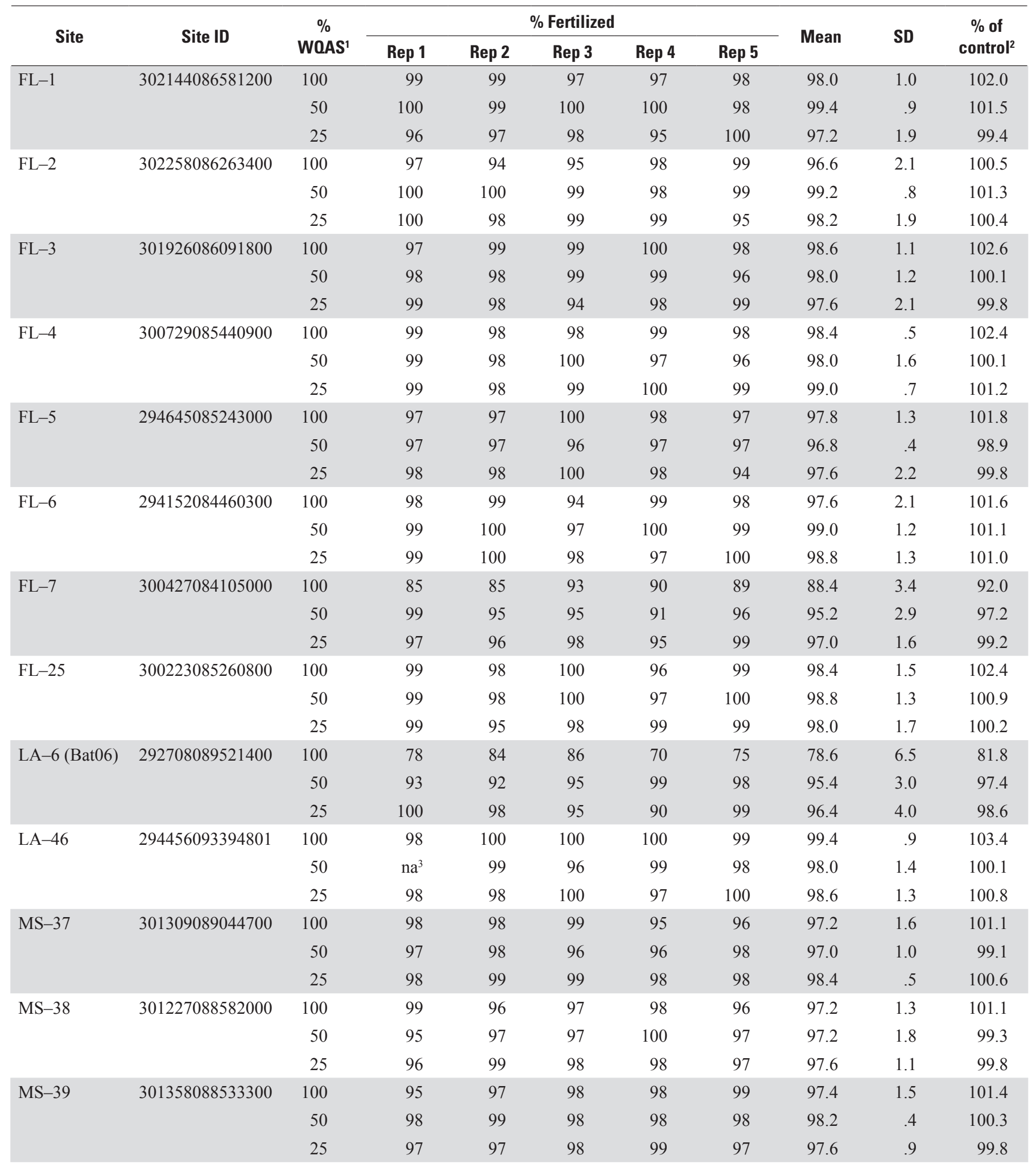


Table 8. Sea urchin fertilization test raw data and means for experiment two with pore-water samples collected from sediments post-landfall of the Deepwater Horizon oil release. - Continued

[Site, U.S. Geological Survey (USGS) identifier for site locality; Site ID, USGS identifier number; \%, percent; WQAS, water quality adjusted sample; Rep, replicate; SD, standard deviation; na, not available; (Rep 2), field replicate two; (Rep 1), field replicate one; MFS, Millipore ${ }^{\circledR}$ filtered seawater; SDS, sodium dodecyl sulfate]

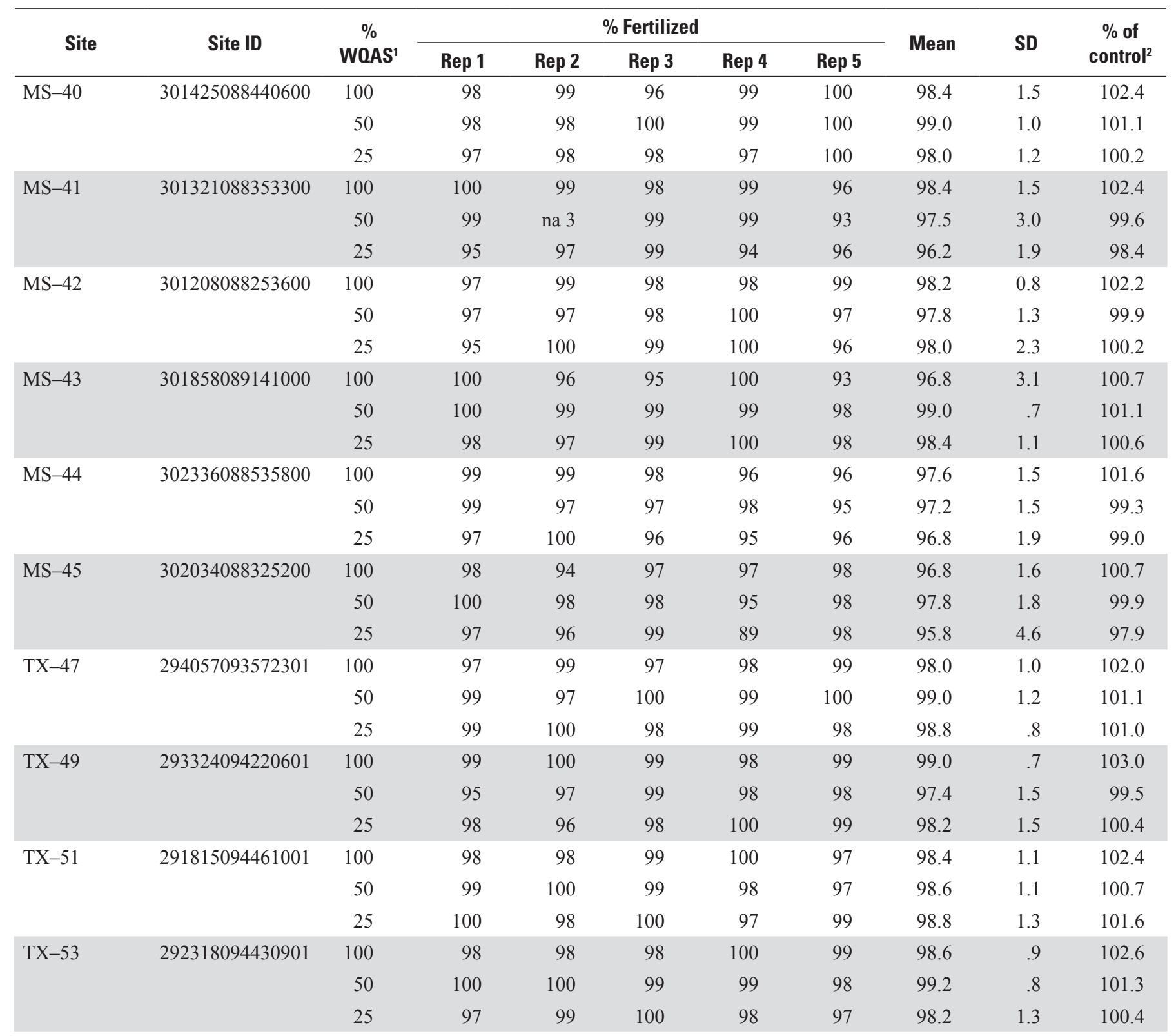


Table 8. Sea urchin fertilization test raw data and means for experiment two with pore-water samples collected from sediments post-landfall of the Deepwater Horizon oil release. - Continued

[Site, U.S. Geological Survey (USGS) identifier for site locality; Site ID, USGS identifier number; \%, percent; WQAS, water quality adjusted sample; Rep, replicate; SD, standard deviation; na, not available; (Rep 2), field replicate two; (Rep 1), field replicate one; MFS, Millipore ${ }^{\circledR}$ filtered seawater; SDS, sodium dodecyl sulfate]

\begin{tabular}{|c|c|c|c|c|c|c|c|c|c|c|}
\hline \multirow{2}{*}{ Site } & \multirow{2}{*}{ Site ID } & \multirow{2}{*}{$\begin{array}{c}\% \\
\text { WQAS }^{1}\end{array}$} & \multicolumn{5}{|c|}{$\%$ Fertilized } & \multirow{2}{*}{ Mean } & \multirow{2}{*}{ SD } & \multirow{2}{*}{$\begin{array}{c}\% \text { of } \\
\text { control }\end{array}$} \\
\hline & & & Rep 1 & Rep 2 & Rep 3 & Rep 4 & $\operatorname{Rep} 5$ & & & \\
\hline \multirow{2}{*}{ TX-55 } & 291251094571401 & 100 & 98 & 99 & 99 & 98 & 96 & 98.0 & 1.2 & 102.0 \\
\hline & & 25 & 99 & 100 & 100 & 99 & 98 & 99.2 & .8 & 101.4 \\
\hline \multirow[t]{2}{*}{ TX-56 } & 290512095063101 & 100 & 99 & 98 & 97 & 96 & 99 & 97.8 & 1.3 & 101.8 \\
\hline & & 25 & 98 & 100 & 98 & 100 & 95 & 98.2 & 2.0 & 100.4 \\
\hline \multirow[t]{4}{*}{ TXREF $^{4}$} & None & 100 & 96 & 97 & 97 & 98 & 97 & 96.1 & 1.8 & 100.0 \\
\hline & & & 96 & 96 & 93 & 93 & 98 & & & \\
\hline & & 50 & 99 & 99 & 96 & 96 & 98 & 97.9 & 1.5 & 100.0 \\
\hline & & & 100 & 97 & 97 & 100 & 97 & & & \\
\hline & & & 95 & 97 & 95 & 97 & 100 & & & \\
\hline Brine blank ${ }^{6}$ & None & & 97 & 97 & 100 & 98 & 98 & 98.0 & 1.2 & 102.0 \\
\hline Rinse blank $^{7}$ & None & 100 & 98 & 97 & 96 & 97 & 98 & 97.2 & .8 & 101.1 \\
\hline \multirow[t]{5}{*}{$\mathrm{SDS}^{8}$} & None & 20 & 0 & 0 & 0 & 0 & 0 & .0 & .0 & .0 \\
\hline & & 10 & 1 & 4 & 4 & 2 & 1 & 2.4 & 1.5 & 2.5 \\
\hline & & 5 & na & 62 & 60 & 60 & 69 & 62.8 & 4.3 & 65.3 \\
\hline & & 2.5 & 96 & 99 & 95 & 97 & 99 & 97.2 & 1.8 & 101.1 \\
\hline & & 1.25 & 100 & 100 & 96 & 99 & 95 & 98.0 & 2.3 & 102.0 \\
\hline
\end{tabular}

${ }^{1}$ Percent of salinity adjusted pore-water sample or concentration of SDS reference toxicant in milligrams per liter.

${ }^{2}$ Percent of TXREF control at the appropriate dilution.

${ }^{3}$ Data point not available because of error in pipetting.

${ }^{4}$ Reference pore water extracted from sediment collected in Aransas Bay, Texas.

${ }^{5}$ Millipore ${ }^{\circledR}$ filtered seawater diluent.

${ }^{6}$ Brine blank consisting of TXREF reference pore water diluted to $6 \%$ and subsequently increased with brine to $30 \%$ with concentrated brine.

${ }^{7}$ Rinsate blank consisting of filtered seawater run over a complete set of all equipment used in pore-water extraction.

${ }^{8}$ Sodium dodecyl sulfate positive control (in milligrams per liter). 
Table 9. Sea urchin embryological development raw data and means for pore-water samples collected from sediments postlandfall of the Deepwater Horizon oil release.

[Site, U.S. Geological Survey (USGS) identifier for site locality; Site ID, USGS identifier number; \%, percent; WQAS, water quality adjusted sample; Rep, replicate; SD, standard deviation; MFS, Millipore ${ }^{\circledR}$ filtered seawater; SDS, sodium dodecyl sulfate]

\begin{tabular}{|c|c|c|c|c|c|c|c|c|c|c|}
\hline \multirow{2}{*}{ Site } & \multirow{2}{*}{ Site ID } & \multirow{2}{*}{$\begin{array}{c}\% \\
\text { W0AS }\end{array}$} & \multicolumn{5}{|c|}{$\%$ Normal Pluteus } & \multirow{2}{*}{ Mean } & \multirow{2}{*}{ SD } & \multirow{2}{*}{$\begin{array}{c}\% \text { of } \\
\text { control }\end{array}$} \\
\hline & & & Rep 1 & Rep 2 & $\operatorname{Rep} 3$ & $\operatorname{Rep} 4$ & Rep 5 & & & \\
\hline \multirow[t]{3}{*}{$\mathrm{AL}-1$} & 301338088193500 & 100 & 96 & 93 & 97 & 96 & 91 & 94.6 & 2.5 & 101.0 \\
\hline & & 50 & 96 & 96 & 96 & 94 & 95 & 95.4 & .9 & 101.2 \\
\hline & & 25 & 94 & 100 & 96 & 96 & 96 & 96.4 & 2.2 & 100.6 \\
\hline \multirow[t]{3}{*}{$\mathrm{AL}-2$} & 301455088110300 & 100 & 97 & 98 & 99 & 96 & 94 & 96.8 & 1.9 & 103.3 \\
\hline & & 50 & 95 & 92 & 95 & 94 & 97 & 94.6 & 1.8 & 100.3 \\
\hline & & 25 & 95 & 95 & 99 & 95 & 93 & 95.4 & 2.2 & 99.6 \\
\hline \multirow[t]{3}{*}{$\mathrm{AL}-3$} & 301448088044000 & 100 & 97 & 93 & 93 & 92 & 93 & 93.6 & 1.9 & 99.9 \\
\hline & & 50 & 90 & 95 & 94 & 98 & 96 & 94.6 & 3.0 & 100.3 \\
\hline & & 25 & 96 & 94 & 94 & 96 & 94 & 94.8 & 1.1 & 99.0 \\
\hline \multirow[t]{3}{*}{$\mathrm{AL}-4$} & 301329088003000 & 100 & 92 & 96 & 94 & 97 & 95 & 94.8 & 1.9 & 101.2 \\
\hline & & 50 & 94 & 93 & 94 & 95 & 96 & 94.4 & 1.1 & 100.1 \\
\hline & & 25 & 94 & 95 & 96 & 95 & 97 & 95.4 & 1.1 & 99.6 \\
\hline \multirow[t]{3}{*}{$\mathrm{AL}-5$} & 301349087541600 & 100 & 94 & 97 & 95 & 93 & 97 & 95.2 & 1.8 & 101.6 \\
\hline & & 50 & 94 & 92 & 96 & 92 & 94 & 93.6 & 1.7 & 99.3 \\
\hline & & 25 & 92 & 97 & 97 & 90 & 98 & 94.8 & 3.6 & 99.0 \\
\hline \multirow[t]{3}{*}{$\mathrm{AL}-6$} & 301428087434900 & 100 & 94 & 93 & 95 & 93 & 95 & 94.0 & 1.0 & 100.3 \\
\hline & & 50 & 96 & 96 & 94 & 97 & 95 & 95.6 & 1.1 & 101.4 \\
\hline & & 25 & 99 & 92 & 95 & 94 & 96 & 95.2 & 2.6 & 99.4 \\
\hline \multirow[t]{3}{*}{$\mathrm{AL}-7$} & 301608087345400 & 100 & 92 & 95 & 95 & 95 & 92 & 93.8 & 1.6 & 100.1 \\
\hline & & 50 & 95 & 95 & 98 & 92 & 95 & 95.0 & 2.1 & 100.7 \\
\hline & & 25 & 93 & 97 & 94 & 91 & 91 & 93.2 & 2.5 & 97.3 \\
\hline \multirow[t]{3}{*}{ AL-8 } & 301353087561600 & 100 & 99 & 97 & 97 & 96 & 95 & 96.8 & 1.5 & 103.3 \\
\hline & & 50 & 95 & 97 & 97 & 93 & 97 & 95.8 & 1.8 & 101.6 \\
\hline & & 25 & 96 & 97 & 96 & 93 & 94 & 95.2 & 1.6 & 99.4 \\
\hline \multirow[t]{3}{*}{ AL-9 } & 301343087520200 & 100 & 94 & 97 & 94 & 96 & 95 & 95.2 & 1.3 & 101.6 \\
\hline & & 50 & 93 & 90 & 94 & 97 & 93 & 93.4 & 2.5 & 99.0 \\
\hline & & 25 & 91 & 95 & 97 & 93 & 96 & 94.4 & 2.4 & 98.5 \\
\hline \multirow[t]{3}{*}{$\mathrm{AL}-10$} & 301341087495200 & 100 & 98 & 96 & 96 & 96 & 93 & 95.8 & 1.8 & 102.2 \\
\hline & & 50 & 97 & 95 & 93 & 97 & 95 & 95.4 & 1.7 & 101.2 \\
\hline & & 25 & 92 & 98 & 91 & 98 & 93 & 94.4 & 3.4 & 98.5 \\
\hline \multirow[t]{3}{*}{ LA-6 (Bat06) } & 292708089521400 & 100 & 97 & 91 & 93 & 92 & 91 & 92.8 & 2.5 & 99.0 \\
\hline & & 50 & 94 & 94 & 95 & 95 & 95 & 94.6 & .5 & 100.3 \\
\hline & & 25 & 95 & 98 & 92 & 96 & 97 & 95.6 & 2.3 & 99.8 \\
\hline \multirow[t]{3}{*}{ LA-22 } & 294432090083100 & 100 & 94 & 97 & 97 & 94 & 91 & 94.6 & 2.5 & 101.0 \\
\hline & & 50 & 92 & 95 & 94 & 96 & 97 & 94.8 & 1.9 & 100.5 \\
\hline & & 25 & 94 & 94 & 92 & 94 & 92 & 93.2 & 1.1 & 97.3 \\
\hline \multirow[t]{3}{*}{ LA-23 } & 294406091511300 & 100 & 90 & 92 & 95 & 99 & 95 & 94.2 & 3.4 & 100.5 \\
\hline & & 50 & 91 & 90 & 97 & 96 & 94 & 93.6 & 3.0 & 99.3 \\
\hline & & 25 & 98 & 95 & 97 & 96 & 96 & 96.4 & 1.1 & 100.6 \\
\hline
\end{tabular}


Table 9. Sea urchin embryological development raw data and means for pore-water samples collected from sediments postlandfall of the Deepwater Horizon oil release. - Continued

[Site, U.S. Geological Survey (USGS) identifier for site locality; Site ID, USGS identifier number; \%, percent; WQAS, water quality adjusted sample; Rep, replicate; SD, standard deviation; MFS, Millipore ${ }^{\circledR}$ filtered seawater; SDS, sodium dodecyl sulfate]

\begin{tabular}{|c|c|c|c|c|c|c|c|c|c|c|}
\hline \multirow{2}{*}{ Site } & \multirow{2}{*}{ Site ID } & \multirow{2}{*}{$\begin{array}{c}\% \\
\text { WOAS }^{1}\end{array}$} & \multicolumn{5}{|c|}{$\%$ Normal Pluteus } & \multirow{2}{*}{ Mean } & \multirow{2}{*}{ SD } & \multirow{2}{*}{$\begin{array}{c}\% \text { of } \\
\text { control }\end{array}$} \\
\hline & & & Rep 1 & Rep 2 & Rep 3 & Rep 4 & Rep 5 & & & \\
\hline \multirow[t]{3}{*}{ LA-24 } & 292046090254500 & 100 & 91 & 88 & 88 & 88 & 87 & 88.4 & 1.5 & 94.3 \\
\hline & & 50 & 94 & 92 & 95 & 97 & 97 & 95.0 & 2.1 & 100.7 \\
\hline & & 25 & 95 & 94 & 96 & 95 & 94 & 94.8 & .8 & 99.0 \\
\hline \multirow[t]{3}{*}{ LA-25 } & 293808092460200 & 100 & 0 & 4 & 2 & 8 & 0 & 2.8 & 3.3 & 3.0 \\
\hline & & 50 & 93 & 91 & 94 & 92 & 93 & 92.6 & 1.1 & 98.2 \\
\hline & & 25 & 97 & 93 & 94 & 95 & 90 & 93.8 & 2.6 & 97.9 \\
\hline \multirow[t]{3}{*}{ LA-26 } & 291507090551800 & 100 & 0 & 0 & 0 & 0 & 0 & 0.0 & .0 & .0 \\
\hline & & 50 & 75 & 86 & 78 & 83 & 89 & 82.2 & 5.7 & 87.2 \\
\hline & & 25 & 96 & 95 & 93 & 93 & 95 & 94.4 & 1.3 & 98.5 \\
\hline \multirow[t]{3}{*}{ LA-28 } & 293424091321600 & 100 & 94 & 95 & 90 & 94 & 96 & 93.8 & 2.3 & 100.1 \\
\hline & & 50 & 94 & 95 & 95 & 93 & 91 & 93.6 & 1.7 & 99.3 \\
\hline & & 25 & 94 & 98 & 95 & 94 & 95 & 95.2 & 1.6 & 99.4 \\
\hline \multirow[t]{3}{*}{ LA-29 } & 294324089432500 & 100 & 93 & 91 & 91 & 92 & 89 & 91.2 & 1.5 & 97.3 \\
\hline & & 50 & 93 & 93 & 94 & 91 & 91 & 92.4 & 1.3 & 98.0 \\
\hline & & 25 & 98 & 95 & 93 & 91 & 95 & 94.3 & 3.0 & 98.4 \\
\hline \multirow[t]{3}{*}{ LA-30 } & 294108089234500 & 100 & 92 & 93 & 94 & 97 & 91 & 93.4 & 2.3 & 99.7 \\
\hline & & 50 & 94 & 93 & 93 & 93 & 94 & 93.4 & .5 & 99.0 \\
\hline & & 25 & 91 & 94 & 95 & 95 & 97 & 94.4 & 2.2 & 98.5 \\
\hline \multirow[t]{3}{*}{ LA -31} & 291537089570100 & 100 & 0 & 0 & 0 & 0 & 0 & .0 & .0 & .0 \\
\hline & & 50 & 93 & 92 & 91 & 82 & 86 & 88.8 & 4.7 & 94.2 \\
\hline & & 25 & 98 & 93 & 96 & 96 & 95 & 95.6 & 1.8 & 99.8 \\
\hline \multirow[t]{3}{*}{ LA-32 } & 291914089105500 & 100 & 97 & 95 & 92 & 91 & 97 & 94.4 & 2.8 & 100.7 \\
\hline & & 50 & 96 & 97 & 95 & 92 & 90 & 94.0 & 2.9 & 99.7 \\
\hline & & 25 & 95 & 97 & 94 & 97 & 94 & 95.4 & 1.5 & 99.6 \\
\hline \multirow[t]{3}{*}{ LA-33 } & 293518089364300 & 100 & 54 & 6 & 8 & 31 & 1 & 20.0 & 22.2 & 21.3 \\
\hline & & 50 & 97 & 93 & 96 & 92 & 88 & 93.2 & 3.6 & 98.8 \\
\hline & & 25 & 97 & 97 & 98 & 92 & 98 & 96.4 & 2.5 & 100.6 \\
\hline \multirow[t]{3}{*}{ LA-34 } & 30090789144500 & 100 & 94 & 95 & 95 & 96 & 94 & 94.8 & .8 & 101.2 \\
\hline & & 50 & 87 & 89 & 95 & 98 & 93 & 92.4 & 4.4 & 98.0 \\
\hline & & 25 & 97 & 95 & 94 & 92 & 93 & 94.2 & 1.9 & 98.3 \\
\hline \multirow[t]{3}{*}{ LA -35} & 285951089085600 & 100 & 93 & 94 & 98 & 92 & 94 & 94.2 & 2.3 & 100.5 \\
\hline & & 50 & 94 & 95 & 95 & 95 & 93 & 94.4 & .9 & 100.1 \\
\hline & & 25 & 98 & 96 & 94 & 96 & 97 & 96.2 & 1.5 & 100.4 \\
\hline \multirow[t]{3}{*}{ LA-36 } & 285615089235600 & 100 & 6 & 20 & 8 & 11 & 5 & 10.0 & 6.0 & 10.7 \\
\hline & & 50 & 96 & 94 & 91 & 96 & 92 & 93.8 & 2.3 & 99.5 \\
\hline & & 25 & 95 & 88 & 96 & 94 & 92 & 93.0 & 3.2 & 97.1 \\
\hline \multirow[t]{3}{*}{ LA -46} & 294456093394801 & 100 & 94 & 97 & 97 & 97 & 98 & 96.6 & 1.5 & 103.1 \\
\hline & & 50 & 94 & 90 & 95 & 97 & 96 & 94.4 & 2.7 & 100.1 \\
\hline & & 25 & 98 & 95 & 97 & 89 & 94 & 94.6 & 3.5 & 98.7 \\
\hline
\end{tabular}


Table 9. Sea urchin embryological development raw data and means for pore-water samples collected from sediments postlandfall of the Deepwater Horizon oil release. - Continued

[Site, U.S. Geological Survey (USGS) identifier for site locality; Site ID, USGS identifier number; \%, percent; WQAS, water quality adjusted sample; Rep, replicate; SD, standard deviation; MFS, Millipore ${ }^{\circledR}$ filtered seawater; SDS, sodium dodecyl sulfate]

\begin{tabular}{|c|c|c|c|c|c|c|c|c|c|c|}
\hline \multirow{2}{*}{ Site } & \multirow{2}{*}{ Site ID } & \multirow{2}{*}{$\begin{array}{c}\% \\
\text { WOAS }\end{array}$} & \multicolumn{5}{|c|}{$\%$ Normal Pluteus } & \multirow{2}{*}{ Mean } & \multirow{2}{*}{ SD } & \multirow{2}{*}{$\begin{array}{c}\% \text { of } \\
\text { control }^{2}\end{array}$} \\
\hline & & & Rep 1 & Rep 2 & Rep 3 & Rep 4 & Rep 5 & & & \\
\hline \multirow[t]{3}{*}{ FL-1 } & 302144086581200 & 100 & 92 & 93 & 91 & 93 & 92 & 92.2 & .8 & 98.4 \\
\hline & & 50 & 95 & 93 & 99 & 90 & 94 & 94.2 & 3.3 & 99.9 \\
\hline & & 25 & 98 & 95 & 89 & 92 & 93 & 93.4 & 3.4 & 97.5 \\
\hline \multirow[t]{3}{*}{ FL-2 } & 302258086263400 & 100 & 99 & 94 & 96 & 91 & 92 & 94.4 & 3.2 & 100.7 \\
\hline & & 50 & 94 & 92 & 93 & 94 & 89 & 92.4 & 2.1 & 98.0 \\
\hline & & 25 & 95 & 92 & 97 & 94 & 94 & 94.4 & 1.8 & 98.5 \\
\hline \multirow[t]{3}{*}{ FL-3 } & 301926086091800 & 100 & 92 & 97 & 91 & 95 & 90 & 93.0 & 2.9 & 99.3 \\
\hline & & 50 & 89 & 94 & 96 & 97 & 92 & 93.6 & 3.2 & 99.3 \\
\hline & & 25 & 93 & 94 & 91 & 90 & 97 & 93.0 & 2.7 & 97.1 \\
\hline \multirow[t]{3}{*}{$\mathrm{FL}-4$} & 300729085440900 & 100 & 95 & 92 & 95 & 94 & 91 & 93.4 & 1.8 & 99.7 \\
\hline & & 50 & 93 & 94 & 96 & 96 & 94 & 94.6 & 1.3 & 100.3 \\
\hline & & 25 & 93 & 93 & 92 & 93 & 95 & 93.2 & 1.1 & 97.3 \\
\hline \multirow[t]{3}{*}{ FL-5 } & 294645085243000 & 100 & 94 & 97 & 92 & 94 & 98 & 95.0 & 2.4 & 101.4 \\
\hline & & 50 & 99 & 93 & 96 & 93 & 97 & 95.6 & 2.6 & 101.4 \\
\hline & & 25 & 92 & 97 & 91 & 94 & 98 & 94.4 & 3.0 & 98.5 \\
\hline \multirow[t]{3}{*}{ FL-6 } & 294152084460300 & 100 & 91 & 93 & 92 & 95 & 96 & 93.4 & 2.1 & 99.7 \\
\hline & & 50 & 95 & 93 & 97 & 97 & 95 & 95.4 & 1.7 & 101.2 \\
\hline & & 25 & 94 & 97 & 95 & 97 & 94 & 95.4 & 1.5 & 99.6 \\
\hline \multirow[t]{3}{*}{ FL-7 } & 300427084105000 & 100 & 94 & 94 & 88 & 95 & 92 & 92.6 & 2.8 & 98.8 \\
\hline & & 50 & 100 & 95 & 93 & 92 & 91 & 94.2 & 3.6 & 99.9 \\
\hline & & 25 & 96 & 95 & 92 & 98 & 90 & 94.2 & 3.2 & 98.3 \\
\hline \multirow[t]{3}{*}{ FL-25 } & 300223085260800 & 100 & 93 & 95 & 95 & 95 & 91 & 93.8 & 1.8 & 100.1 \\
\hline & & 50 & 91 & 91 & 93 & 94 & 92 & 92.2 & 1.3 & 97.8 \\
\hline & & 25 & 89 & 99 & 96 & 94 & 97 & 95.0 & 3.8 & 99.2 \\
\hline \multirow[t]{3}{*}{ MS-37 } & 301309089044700 & 100 & 97 & 92 & 91 & 95 & 95 & 94.0 & 2.4 & 100.3 \\
\hline & & 50 & 95 & 98 & 96 & 95 & 96 & 96.0 & 1.2 & 101.8 \\
\hline & & 25 & 97 & 95 & 99 & 95 & 94 & 96.0 & 2.0 & 100.2 \\
\hline \multirow[t]{3}{*}{ MS-38 } & 301227088582000 & 100 & 90 & 91 & 94 & 90 & 94 & 91.8 & 2.0 & 98.0 \\
\hline & & 50 & 93 & 89 & 96 & 87 & 91 & 91.2 & 3.5 & 96.7 \\
\hline & & 25 & 95 & 97 & 94 & 97 & 93 & 95.2 & 1.8 & 99.4 \\
\hline \multirow[t]{3}{*}{ MS-39 } & 301358088533300 & 100 & 0 & 4 & 1 & 5 & 7 & 3.4 & 2.9 & 3.6 \\
\hline & & 50 & 92 & 92 & 91 & 87 & 93 & 91.0 & 2.3 & 96.5 \\
\hline & & 25 & 94 & 96 & 90 & 94 & 96 & 94.0 & 2.4 & 98.1 \\
\hline \multirow[t]{3}{*}{ MS-40 } & 301425088440600 & 100 & 94 & 93 & 96 & 94 & 95 & 94.4 & 1.1 & 100.7 \\
\hline & & 50 & 92 & 92 & 98 & 94 & 96 & 94.4 & 2.6 & 100.1 \\
\hline & & 25 & 95 & 94 & 96 & 93 & 97 & 95.0 & 1.6 & 99.2 \\
\hline \multirow[t]{3}{*}{ MS-41 } & 301321088353300 & 100 & 92 & 95 & 94 & 95 & 92 & 93.6 & 1.5 & 99.9 \\
\hline & & 50 & 93 & 94 & 93 & 95 & 94 & 93.8 & .8 & 99.5 \\
\hline & & 25 & 98 & 94 & 95 & 95 & 98 & 96.0 & 1.9 & 100.2 \\
\hline
\end{tabular}


Table 9. Sea urchin embryological development raw data and means for pore-water samples collected from sediments postlandfall of the Deepwater Horizon oil release. - Continued

[Site, U.S. Geological Survey (USGS) identifier for site locality; Site ID, USGS identifier number; \%, percent; WQAS, water quality adjusted sample; Rep, replicate; SD, standard deviation; MFS, Millipore ${ }^{\circledR}$ filtered seawater; SDS, sodium dodecyl sulfate]

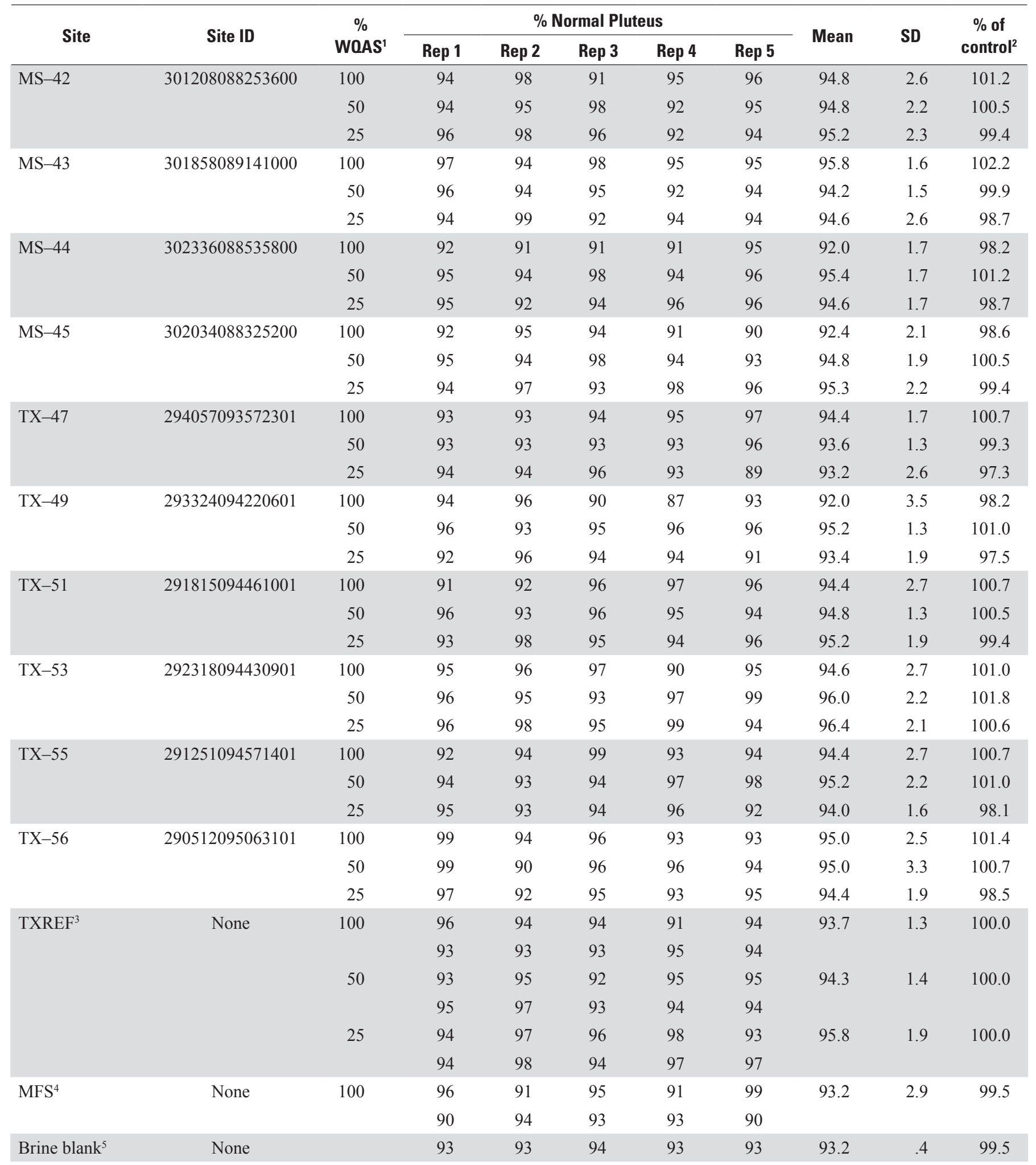


Table 9. Sea urchin embryological development raw data and means for pore-water samples collected from sediments postlandfall of the Deepwater Horizon oil release. - Continued

[Site, U.S. Geological Survey (USGS) identifier for site locality; Site ID, USGS identifier number; \%, percent; WQAS, water quality adjusted sample; Rep, replicate; SD, standard deviation; MFS, Millipore ${ }^{\circledR}$ filtered seawater; SDS, sodium dodecyl sulfate]

\begin{tabular}{|c|c|c|c|c|c|c|c|c|c|c|}
\hline \multirow{2}{*}{ Site } & \multirow{2}{*}{ Site ID } & \multirow{2}{*}{$\begin{array}{c}\% \\
\text { WOAS }\end{array}$} & \multicolumn{5}{|c|}{$\%$ Normal Pluteus } & \multirow{2}{*}{ Mean } & \multirow{2}{*}{ SD } & \multirow{2}{*}{$\begin{array}{c}\% \text { of } \\
\text { control }\end{array}$} \\
\hline & & & Rep 1 & Rep 2 & Rep 3 & Rep 4 & Rep 5 & & & \\
\hline Rinse blank ${ }^{6}$ & None & 100 & 94 & 96 & 98 & 91 & 94 & 94.6 & 2.6 & 101.0 \\
\hline \multirow[t]{5}{*}{$\operatorname{SDS}^{7}$} & None & 20 & 0 & 0 & 0 & 0 & 0 & 0.0 & 0.0 & 0.0 \\
\hline & & 10 & 0 & 0 & 0 & 0 & 0 & .0 & .0 & .0 \\
\hline & & 5 & 5 & 3 & 5 & 4 & 1 & 3.6 & 1.7 & 3.8 \\
\hline & & 2.5 & 91 & 88 & 87 & 79 & 86 & 86.2 & 4.4 & 92.0 \\
\hline & & 1.25 & 96 & 93 & 94 & 95 & 97 & 95.0 & 1.6 & 101.4 \\
\hline
\end{tabular}

${ }^{1}$ Percent of water quality adjusted pore water sampled or concentration of SDS reference toxicant in milligrams per liter.

${ }^{2}$ Percent of TXREF control at the appropriate dilution.

${ }^{3}$ Reference pore water extracted from sediment collected in Aransas Bay, Texas.

${ }^{4}$ Millipore ${ }^{\circledR}$ filtered seawater diluent.

${ }^{5}$ Brine blank consisting of TXREF reference pore water diluted to $6 \%$ and subsequently increased with brine to $30 \%$ with concentrated brine.

${ }^{6}$ Rinsate blank consisting of filtered seawater run over a complete set of all equipment used in pore-water extraction.

${ }^{7}$ Sodium dodecyl sulfate positive control (in milligrams per liter).

Table 10. Water-quality measurements after salinity adjustment and original salinity of pore-water samples collected from sediments post-landfall of the Deepwater Horizon oil release and tested in the Toxicity Identification Evaluation (TIE).

[Site, U.S. Geological Survey (USGS) identifier for site locality; Site ID, USGS identifier number; \%o, parts per thousand; \%, percent; DO, dissolved oxygen; $\mathrm{mg} / \mathrm{L}$, milligrams per liter; TAN, total ammonia as nitrogen; UAN, un-ionized ammonia as nitrogen; $\mu \mathrm{g} / \mathrm{L}$, micrograms per liter; OS, original sample; <, less than; MFS, Millipore ${ }^{\circledR}$ filtered seawater; \pm , plus or minus]

\begin{tabular}{|c|c|c|c|c|c|c|c|c|c|}
\hline Site & Site ID & $\begin{array}{c}\text { Salinity } \\
\% 0\end{array}$ & $\begin{array}{c}\text { DO } \\
\text { (mg/L) }\end{array}$ & $\%$ DO & $\mathrm{pH}$ & $\begin{array}{l}\text { TAN } \\
\text { (mg/L) }\end{array}$ & $\begin{array}{l}\text { UAN } \\
\text { ( } \mu \mathrm{g} / \mathrm{L})\end{array}$ & $\begin{array}{l}\text { Sulfide } \\
\text { (mg/L) }\end{array}$ & $\% \mathbf{O S}^{2}$ \\
\hline LA-29 & 294324089432500 & 6 & 7.67 & 104.6 & 8.13 & 0.906 & 38.1 & 0.040 & 75.0 \\
\hline LA-31 & 291537089570100 & 27 & 7.34 & 98.5 & 8.05 & 2.72 & 94.7 & $<.009$ & 96.2 \\
\hline LA-33 & 293518089364300 & 9 & 7.81 & 105.7 & 8.33 & 1.41 & 92.0 & .009 & 77.7 \\
\hline LA-36 & 285615089235600 & 18 & 7.59 & 103 & 7.55 & .903 & 10.3 & $<.009$ & 85.7 \\
\hline MS-39 & 301358088533300 & 29 & 6.69 & 90.4 & 8.05 & .742 & 26.2 & $<.009$ & 100.0 \\
\hline TXREF $^{3}$ & None & 25 & 7.31 & 98.7 & 8.30 & .587 & 35.4 & $<.009$ & 93.6 \\
\hline $\mathrm{MFS}^{4}$ & None & 32 & 6.87 & 92.8 & 8.17 & $<.1$ & $<4.6$ & $<.009$ & 93.8 \\
\hline Brine blank & None & 25 & 6.13 & 84.2 & 8.25 & .183 & 10.0 & $<.009$ & 18.1 \\
\hline
\end{tabular}

${ }^{1}$ Salinty of sample before adjustment. Sample adjusted to $30 \pm 1 \%$.

${ }^{2}$ Percent of original sample after salinity adjustment.

${ }^{3}$ Reference pore water extracted from sediment collected in Aransas Bay, Texas.

${ }^{4}$ Millipore ${ }^{\circledR}$ filtered seawater diluent.

${ }^{5}$ Brine blank of TXREF diluted to $6 \%$ with Milli-Q ${ }^{\circledR}$ purified water and subsequently increased with brine to $30 \%$ (concentrated brine at $102 \%$ ). 
Table 11. Toxicity Identification evaluation (TIE) fertilization raw data ans means for pore-water samples identified as toxic in the Deep Water Horizon post-landfall sea urchin fertilization test.

[Site, U.S. Geological Survey (USGS) identifier for site locality; Site ID, USGS identifier number; \%, percent; Rep, replicate; SD, stansard deviation; Sig, significance; na, not applicable; EDTA, Disodium Ethylenediamine Tetraacetate; **, significant reduction in toxicity; ns, not significant; MFS, Millipore ${ }^{\circledR}$ filtered seawater; SDS, sodium dodecyl sulfate; $\mathrm{mg} / \mathrm{L}$, milligrams per liter; $\mathrm{mg} / \mathrm{mL}$, milligrams per milliliter]

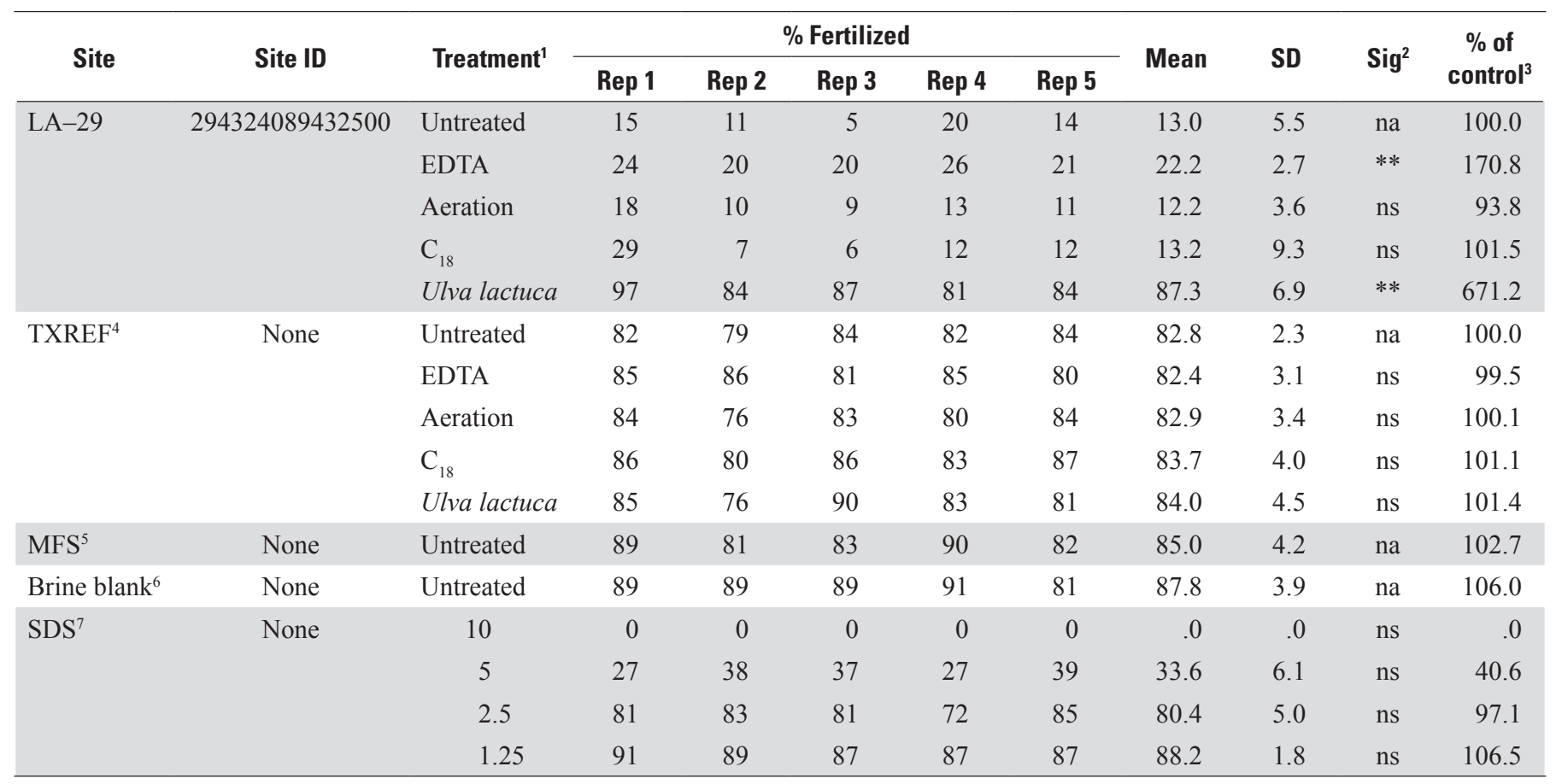

${ }^{1}$ Manipulations performed on sample to reduce toxicity including: no treatment (untreated); addition of EDTA $2 \mathrm{H}_{2} 0$ (sodium salt) at $60 \mathrm{mg} / \mathrm{L}$ for $3 \mathrm{hours}$; vigorous aeration for 1 hour; $\mathrm{C}_{18}$ solid phase extraction (SPE); ans 5 hour exposure of sample to the algae Ulva lactuca at $83.3 \mathrm{mg} / \mathrm{mL}$ or concentration in milligrams per liter in the case of the SDS positive control.

${ }^{2}$ Significance in relation to the untreated sample; Dunnetts $t$-test $\alpha<0.01$.

${ }^{3}$ Percent of the control (in this case) the untreated sample or in the case of MFS, brine blank, ans SDS the untreated TXREF control.

${ }^{4}$ Reference pore water extracted from sediment collected in Aransas Bay, Texas.

${ }^{5}$ Millipore ${ }^{\circledR}$ filtered seawater diluent.

${ }^{6}$ Brine blank consisting of TXREF reference pore water diluted to $6 \%$ ans subsequently increased with brine to $30 \%$ with concentrated brine.

${ }^{7}$ Sodium dodecyl sulfate positive control (in milligrams per liter). 
Table 12. Toxicity Identification evaluation (TIE) embryological development raw data and means for pore-water samples identified as toxic in the Deep Water Horizon post-landfall sea urchin embryological development test.

[Site, U.S. Geological Survey (USGS) identifier for site locality; Site ID, USGS identifier number; \%, percent; Rep, replicate; SD, standard deviation; Sig, significance; na, not applicable; EDTA, Disodium Ethylenediamine Tetraacetate; ns, not significant; **, no significant reduction in toxicity; ++, significant increase in toxicity; MFS, Millipore ${ }^{\circledR}$ filtered seawater; SDS, sodium dodecyl sulfate; mg/L, milligrams per liter; mg/mL, milligrams per milliliter; $\mu \mathrm{g} / \mathrm{L}$, micrograms per liter]

\begin{tabular}{|c|c|c|c|c|c|c|c|c|c|c|c|}
\hline \multirow{2}{*}{ Site } & \multirow{2}{*}{ Site ID } & \multirow{2}{*}{ Treatment $^{1}$} & \multicolumn{5}{|c|}{$\%$ Normal Pluteus } & \multirow{2}{*}{ Mean } & \multirow{2}{*}{ SD } & \multirow{2}{*}{$\mathrm{Sig}^{2}$} & \multirow{2}{*}{$\begin{array}{c}\% \text { of } \\
\text { control }\end{array}$} \\
\hline & & & Rep 1 & Rep 2 & Rep 3 & Rep 4 & Rep 5 & & & & \\
\hline \multirow{5}{*}{ LA-31 } & 291537089570100 & Untreated & 0 & 1 & 0 & 2 & 0 & 0.6 & 0.9 & na & 100.0 \\
\hline & & EDTA & 4 & 12 & 0 & 8 & 2 & 5.2 & 4.8 & ns & 866.7 \\
\hline & & Aeration & 0 & 0 & 0 & 0 & 0 & 0 & .0 & ns & .0 \\
\hline & & $\mathrm{C}_{18}$ & 0 & 0 & 0 & 0 & 2 & .4 & .9 & ns & 66.7 \\
\hline & & Ulva lactuca & 87 & 91 & 91 & 91 & 88 & 89.3 & 2.1 & $* *$ & $14,875.0$ \\
\hline \multirow[t]{5}{*}{ LA-33 } & 293518089364300 & Untreated & 0 & 2 & 8 & 0 & 8 & 3.6 & 4.1 & na & 100.0 \\
\hline & & EDTA & 29 & 19 & 39 & 45 & 29 & 32.2 & 10.1 & $* *$ & 894.4 \\
\hline & & Aeration & 0 & 0 & 0 & 0 & 0 & .0 & .0 & ns & .0 \\
\hline & & $\mathrm{C}_{18}$ & 8 & 14 & 50 & 10 & 20 & 20.4 & 17.2 & $\mathrm{~ns}$ & 566.7 \\
\hline & & Ulva lactuca & 88 & 94 & 93 & 93 & 86 & 90.0 & 3.6 & $* *$ & $2,500.0$ \\
\hline \multirow[t]{5}{*}{ LA-36 } & 285615089235600 & Untreated & 78 & 78 & 75 & 80 & 77 & 77.6 & 1.8 & na & 100.0 \\
\hline & & EDTA & 93 & 87 & 95 & 86 & 94 & 91.0 & 4.2 & $* *$ & 117.3 \\
\hline & & Aeration & 64 & 58 & 78 & 61 & 70 & 66.2 & 7.9 & ++ & 85.3 \\
\hline & & $\mathrm{C}_{18}$ & 81 & 76 & 85 & 81 & 81 & 80.8 & 3.2 & ns & 104.1 \\
\hline & & Ulva lactuca & 91 & 92 & 90 & 93 & 90 & 91.0 & 1.4 & $* *$ & 117.3 \\
\hline \multirow[t]{5}{*}{ MS-39 } & 301358088533300 & Untreated & 15 & 29 & 28 & 23 & 31 & 25.2 & 6.4 & na & 100.0 \\
\hline & & EDTA & 84 & 79 & 58 & 76 & 86 & 76.6 & 11.1 & $* *$ & 304.0 \\
\hline & & Aeration & 8 & 19 & 2 & 31 & 17 & 15.4 & 11.1 & ns & 61.1 \\
\hline & & $\mathrm{C}_{18}$ & 93 & 90 & 91 & 89 & 94 & 91.4 & 2.1 & $* *$ & 362.7 \\
\hline & & Ulva lactuca & 95 & 94 & 96 & 99 & 98 & 97.0 & 1.8 & $* *$ & 384.9 \\
\hline $\mathrm{MFS}^{4}$ & None & Untreated & 95 & 96 & 95 & 92 & 95 & 94.6 & 1.5 & na & 99.2 \\
\hline Brine blank ${ }^{5}$ & None & Untreated & 93 & 95 & 100 & 97 & 100 & 97.0 & 3.1 & na & 101.7 \\
\hline \multirow[t]{5}{*}{ TXREF $^{6}$} & None & Untreated & 97 & 95 & 95 & 96 & 94 & 95.4 & 1.4 & na & 100.0 \\
\hline & & EDTA & 98 & 96 & 93 & 95 & 95 & 95.8 & 1.6 & ns & 100.4 \\
\hline & & Aeration & 96 & 97 & 94 & 96 & 98 & 95.7 & 1.6 & ns & 100.3 \\
\hline & & $\mathrm{C}_{18}$ & 96 & 96 & 94 & 97 & 93 & 94.1 & 2.7 & $\mathrm{~ns}$ & 98.6 \\
\hline & & Ulva lactuca & 96 & 96 & 92 & 93 & 88 & 93.0 & 3.3 & $\mathrm{~ns}$ & 97.5 \\
\hline \multirow[t]{4}{*}{$\mathrm{SDS}^{7}$} & None & 10 & 0 & 0 & 0 & 0 & 0 & .0 & .0 & $\mathrm{~ns}$ & .0 \\
\hline & & 5 & 0 & 0 & 0 & 0 & 0 & .0 & .0 & $\mathrm{~ns}$ & .0 \\
\hline & & 2.5 & 82 & 75 & 85 & 77 & 82 & 80.2 & 4.1 & ns & 84.1 \\
\hline & & 1.25 & 94 & 90 & 96 & 94 & 93 & 93.4 & 2.2 & $\mathrm{~ns}$ & 97.9 \\
\hline \multirow[t]{4}{*}{$\mathrm{NH}_{3}^{8}$} & None & 129.8 & 0 & 0 & 0 & 0 & 0 & .0 & .0 & $\mathrm{~ns}$ & .0 \\
\hline & & 68.1 & 5 & 1 & 2 & 4 & 1 & 2.6 & 1.8 & ns & 2.7 \\
\hline & & 34 & 93 & 95 & 95 & 96 & 93 & 94.4 & 1.3 & $\mathrm{~ns}$ & 99.0 \\
\hline & & 19.2 & 95 & 98 & 94 & 93 & 97 & 95.4 & 2.1 & ns & 100.0 \\
\hline
\end{tabular}

\footnotetext{
${ }^{1}$ Manipulations performed on sample to reduce toxicity including: no treatment (untreated); addition of EDTA $2 \mathrm{H}_{2} 0$ (sodium salt) at $60 \mathrm{mg} / \mathrm{L}$ for $3 \mathrm{hours}$; vigorous aeration for one hour; $\mathrm{C}_{18}$ solid phase extraction (SPE); and 5 hour exposure of sample to the algae Ulva lactuca at $83.3 \mathrm{mg} / \mathrm{mL}$ or concentration in $\mathrm{mg} / \mathrm{L}$ in the case of the SDS positive control or $\mu \mathrm{g} / \mathrm{L}$ in the case of $\mathrm{NH}_{3}$ series.

${ }^{2}$ Significance in relation to the untreated sample; Dunnetts $t$-test $\alpha<0.01$.

${ }^{3}$ Percent of the control in this case the untreated sample or in the case of MFS, brine blank, and SDS the untreated TXREF control.

${ }^{4}$ Millipore ${ }^{\circledR}$ filtered seawater diluent.

${ }^{5}$ Brine blank consisting of TXREF reference pore water diluted to $6 \%$ and subsequently increased with brine to $30 \%$ with concentrated brine.

${ }^{6}$ Reference pore water extracted from sediment collected in Aransas Bay, Texas.

${ }^{7}$ Sodium dodecyl sulfate positive control (in $\mathrm{mg} / \mathrm{L}$ ).

${ }^{8}$ Un-ionized ammonia calculated from measured total ammonia, $\mathrm{pH}$, and temperature (in $\mu \mathrm{g} / \mathrm{L}$ ).
} 


\section{Appendixes}


Date Prepared: May 5, 1990

Date Revised: July 18, 2007

\section{EXTRACTION AND STORAGE OF PORE-WATER SAMPLES}

\subsection{OBJECTIVE}

This protocol describes a procedure for extracting and storing pore-water samples from marine, estuarine, or freshwater sediments for use in toxicity testing. A pressurized extraction device is used to force the pore water from sediment samples. This procedure may be performed in the laboratory or it may be performed at or near the site of sample collection since the sampling apparatus is portable.

\subsection{PREPARATION}

\subsection{Description of the Pore-water Extraction System}

In earlier studies (Carr et al., 1989; Carr and Chapman, 1992) pore water was extracted from sediments using a device constructed of Teflon ${ }^{\circledR}$. Since then, the design has been improved (Carr and Chapman, 1994) The polyvinyl chloride (PVC) extractors in current use are less costly to construct and easier to operate. This device has been used in numerous sediment quality assessment surveys (Carr, et al., 1996a, 1996b, 1996c, 2000, 2001).

The extractor is constructed from a PVC compression coupling for 4" I.D. schedule 40 PVC pipe. These commercially-available couplings (Lascotite ${ }^{\circledR}$ ) consist of a cylinder (25 cm height and $13 \mathrm{~cm}$ diameter) with threaded ends and threaded open compression nuts (Figure 1-1). The coupling is fitted with end plates cut from 7/16" thick PVC sheeting that are held in place by the threaded end nuts. The gaskets provided with the coupling are discarded and silicon O-rings are used to seal the top and bottom connections. The top end plate is fitted with a quick-release fitting where the pressurized air is supplied, and a safety pressure relief valve. Like the original Teflon ${ }^{\circledR}$ extractor, the bottom end plate (Figure 1-1) has several interconnected concentric grooves to facilitate flow of the pore water to the central exit port. A $5 \mu \mathrm{m}$ polyester filter is situated between the bottom end plate and the silicon O-ring. Before a sediment sample is loaded, the bottom end nut is tightened in place by using the stationary bottom wrench (Figure 1-1) and a standard strap wrench. 


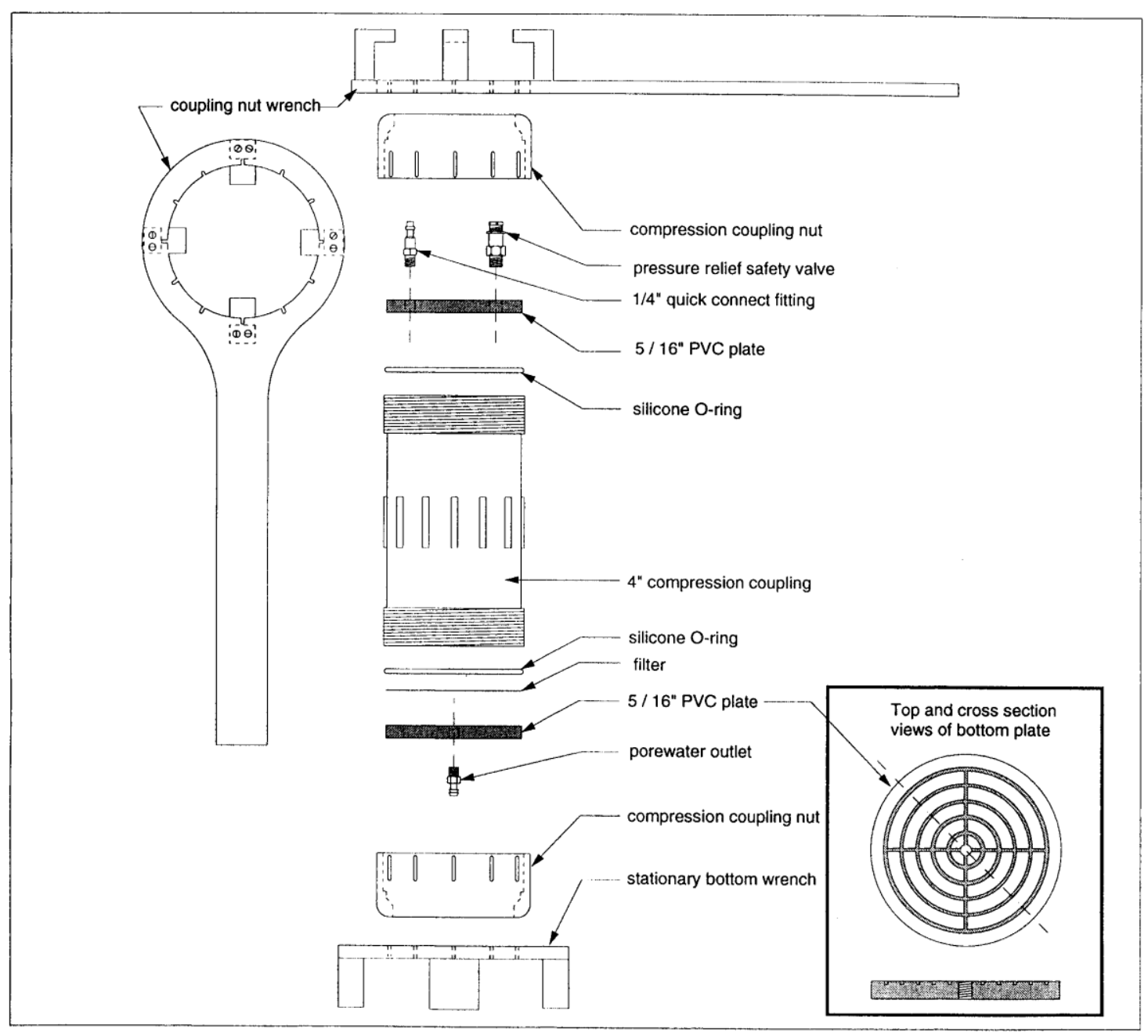

Figure 1-1. Schematic of sediment pore water squeeze extraction device.

The extractors are pressurized with air supplied from a standard SCUBA cylinder via a SCUBA first stage regulator which delivers air to a manifold with a valving system 
(Figure 1-2). With this system, multiple cylinders can be pressurized simultaneously, using the same SCUBA cylinder.

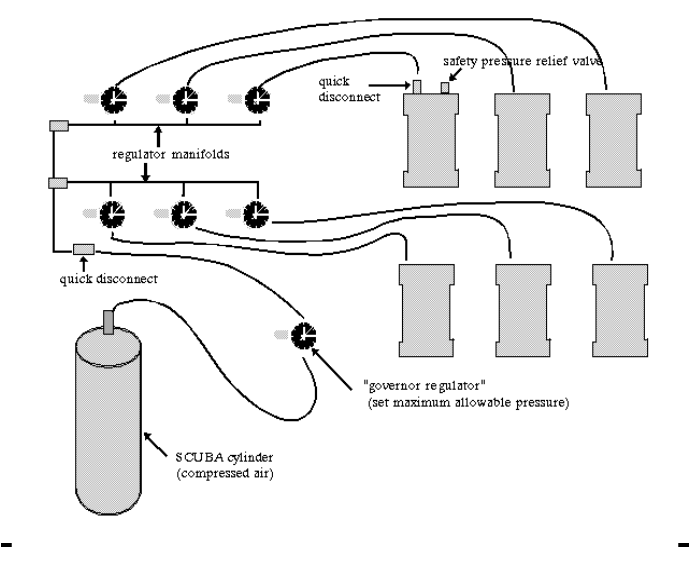

Figure 1-2. Schematic of sediment pore-water pressure extraction system.

\subsection{Equipment List}

Supplies and equipment needed are listed in Attachment A.

\subsection{PROCEDURE}

\subsection{Sediment Collection and Storage Considerations}

Generally, surficial sediment samples are collected for pore-water extraction. A homogenate of the upper $-2-10 \mathrm{~cm}$ sediment may be collected by multiple cores or grabs at a particular sampling station. (Further details of sediment sampling procedures are not within the scope of this SOP.) One liter of sediment will typically provide 100-200 mL pore water. However, a larger volume of coarse sand sediments may be required since they contain less water, and a larger volume of fine clay sediments may be required since they are difficult to extract. The sample composites are kept in suitable containers (e.g., clean high density polyethylene containers or Zip-Lock ${ }^{\circledR}$ bags), labeled, and stored on ice, in a cooler, or in a refrigerator until the samples are delivered and processed. Pore water should be extracted from the samples as soon as possible because the toxicity of sediments in storage may change over time. A sample tracking system should be maintained for each sediment sample collected and pore-water sample extracted. All manipulations made on samples are recorded on the Sample History Data Form (Attachment B). 


\subsection{Load Extraction Cylinder}

1. Assemble all parts of extraction cylinder except the top end compression coupling nut, top end plate and O-ring. Make sure filter is snugly in place beneath bottom O-ring (both over- and under-tightening will result in an improper seal). Place the extractor cylinder on the stand and position an appropriately labeled pore-water sample container (usually an I-Chem ${ }^{\circledR}$ amber $250 \mathrm{~mL}$ or $125 \mathrm{~mL}$ glass jar cleaned to EPA standards, with Teflon ${ }^{\circledR}$ lid liner) underneath the outlet.

2. Ensure that the sediment sample is homogenized, by shaking, stirring with a clean Teflon $^{\circledR}$ or plastic spatula or spoon, or by both.

3. Transfer sediment from the sample container/bag to the extractor by pouring and/or using a clean Teflon ${ }^{\circledR}$ or plastic spatula or spoon. If necessary, particularly when extracting pore water from sandy or shelly sediments, the spatula may be used to compress the sample in the cylinder to eliminate channelization. The amount of sediment to be transferred will depend on the texture of the sample. The cylinder may be filled nearly full with a sandy sediment. However, when extracting pore water from a clay sediment, a relatively impermeable layer of compressed clay will eventually form on the filter, so that extraction of a large volume of clay sediment at once would take an extremely long time. When extracting pore water from extremely fine grained sediments, the cylinder should be less than one-third filled. If additional pore water is needed, this process can be repeated by removing the sediment including removing or "peeling" the impermeable layer, and reintroducing more of the original sediment sample.

4. After sediment is loaded, the top end plate within the top compression coupling nut is installed. To tighten the top nut, the strap wrench and the coupling nut wrench (Figure 1-1) are used.

\subsection{Pore-water Extraction}

After the extractor is sealed, a high-pressure hose is attached to the quick disconnect fitting on the top end plate, and the extractor is pressurized with air from a SCUBA tank. Pressure is controlled with a first-stage regulator on the SCUBA tank, an intermediate "governor" regulator, and final second stage regulators attached to a manifold that services multiple extractors (Figure 1-2).

1. Turn the SCUBA valve counter clockwise, pressurizing the first stage regulator and the intermediate-pressure hose (approximately 150 pounds per square inch (psi)). An additional "governor" pressure regulator between the SCUBA tanks and the final second stage regulators which control pressure to the individual extractors should be set at maximum extractor pressure $(-40 \mathrm{psi})$. 
2. Ensure that all final pressure regulators are set to zero. Attach the hose from one of the pressure regulators on the pressure regulator manifold to the air inlet, using the quick disconnect fitting.

3. Slowly open the corresponding pressure regulator to a pressure of 5-10 psi. Check the first drops of pore water passing from the outlet for cloudiness. Occasionally, a small amount of sediment will pass through the pore-water outlet, presumably around the filter. If this happens, wait until the pore water clears, discard the initial pore water collected, and continue.

4. Check the cylinder for leaks and if necessary tighten clamping nuts slightly.

5. As the flow of pore water decreases, pressure may be increased gradually to a maximum of 35-40 psi. When flow is less than or slows to less than 1-3 drops per minute, increase the pressure in 5-10 psi increments to maintain the flow. Allow the extraction to continue until sufficient pore water has been collected.

6. Disassemble the extractor, discard sediment, and rinse and wash appropriately all parts contacting sediment before placing a different sediment sample into the extractor.

7. Repeat these procedures until all available extractors are in use or until all sediment samples have been processed.

\subsection{Centrifugation of Pore-water Samples}

Pore-water samples extracted at this field station are usually stored frozen until tested. Under most circumstances, the pore-water samples are centrifuged after they are collected and before they are frozen.

1. After collection, keep the pore-water samples refrigerated or chilled on ice until they are centrifuged.

2. Transfer the pore water from the glass sample jar to an appropriate centrifuge bottle (e.g., polycarbonate). Centrifuge at greater than or equal to1,200 times gravity $(\mathrm{g}$ ) for 20 minutes. Return the centrifuged sample to a rinsed and labeled glass jar, taking care not to disturb any material that may have settled on the bottom/sides of the centrifuge bottle.

3. If multiple jars of pore water were collected from a single sediment sample, they should be composited after centrifugation and redistributed to the glass jars before testing or storage. 


\subsection{Storage of Pore-water Samples}

If the pore-water samples are not to be used on the day of collection, they should be frozen for storage. Sufficient room for freeze expansion should be left in the jars (for example, $200 \mathrm{~mL}$ maximum sample in a $250 \mathrm{~mL}$ jar). If the volume needed for testing is known in advance, it is prudent to allocate only that specific volume plus a little excess $(\sim 10 \mathrm{~mL})$ to each jar in order to conserve pore water (once thawed, the pore water cannot be refrozen and reused), and to simplify the volume measurements required for Water Quality Adjustment of Samples (CERC SOP P.651) performed the day prior to testing. Frozen pore-water samples may be shipped with dry ice.

\subsection{QUALITY CONTROL}

A sample tracking system is maintained for each sediment sample collected and pore-water sample extracted. All actions taken with that respective sample are recorded on the Sample History Data Form (Attachment B). This information includes, but not exclusively, : a) the date of collection or receipt, b) the date of pore-water extraction, c) the volume or number of jars (I-Chem ${ }^{\circledR}$ amber glass jars) of pore water collected, d) centrifugation information, if performed, e) date frozen and location (freezer no.), and e) date and jar no. thawed and used in which test. The Sample History Forms are kept in a three-ring binder at the same location where the samples are stored.

\subsection{TRAINING}

Persons who will perform this procedure should first read this SOP and then operate under the supervision of an experienced individual for at least one series of extractions.

\subsection{SAFETY}

The sediment and pore-water samples handled may contain contaminants. Care should be taken to avoid contact with the samples. Protective gloves, glasses and clothing may be worn. Waste sediment should be properly disposed. SCUBA cylinders should be securely mounted before, during, and after use. The pressure limit (40 psi) of the extraction cylinders should not be exceeded. Before disconnecting any pressure hoses, ensure that the pressure has been released or that the controlling regulator has been closed.

\subsection{ATTACHMENTS}

Attachment 1. Required Equipment and Materials

Attachment 2. Sample History Form 


\subsection{REFERENCES}

Carr, R.S., D.C. Chapman, C.L. Howard, and J. Biedenbach. 1996a. Sediment Quality Triad assessment survey in the Galveston Bay Texas system. Ecotoxicology 5:341-361.

Carr, R.S., D.C. Chapman, B.J. Presley, J.M. Biedenbach, L. Robertson, P. Boothe, R. Kilada, T. Wade and P. Montagna. 1996b. Sediment pore-water toxicity assessment studies in the vicinity of offshore oil and gas production platforms in the Gulf of Mexico. Can . J. Fish. Aq. Sci. 53:2618-2628.

Carr, R.S., E.R. Long., D.C. Chapman, G. Thursby, J.M. Biedenbach, H. Windom, G. Sloane and D.A. Wolfe. 1996c. Toxicity assessment studies of contaminated sediments in Tampa Bay, Florida. Environ. Toxicol. Chem. 15:1218-1231.

Carr, R.S., P.A. Montagna, J.M. Biedenbach, R. Kalke, M.C. Kennicutt, R. Hooten, and G. Cripe. 2000. Impact of storm water outfalls on sediment quality in Corpus Christi Bay, Texas.

Environ. Toxicol. Chem. 19:561-574.

Carr, R.S., J.M. Biedenbach, and R. Hooten. 2001. Sediment pore-water toxicity test survey and phase I sediment toxicity identification evaluation studies in Lavaca Bay, Texas - an estuarine Superfund site. Environ. Toxicol. 16:20-30. 
43 Sediment Pore-Water Toxicity Test Results, Deepwater Horizon Oil Release, Gulf of Mexico, 2010 Appendix 1

Prepared by:

Approved by:

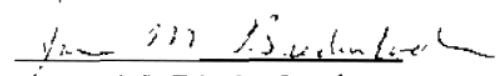

James M. Biedenbach

Biologist

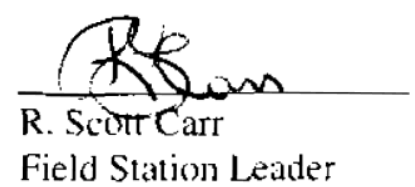

$\frac{\text { (fivivetas }}{\text { Laverne Cleveland }}$

Chief, Field Stations Research Branch

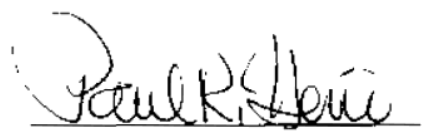

Paul Heine

Quality Assurance Officer 


\section{$\underline{\text { Attachment A }}$}

\section{REQUIRED EQUIPMENT AND MATERIALS}

To construct a sediment pore water extraction device:

1-PVC cylinder (center portion of 4" compression coupling)

2-PVC end nuts (ends of 4 " compression fitting)

1-PVC top end plate (7/16" width)

1-PVC bottom end plate (7/16" width)

1-Quick disconnect brass air fitting

1-Pressure relief valve

1 -Teflon ${ }^{\circledR} 1 / 8^{\prime \prime}$ npt male connector for exit port

To use a pore water extraction device:

1-Filter, polyester material, $5 \mu \mathrm{m}$ pore size

1-Wooden stand ( 1 stand per 3 cylinders)

1-Custom wrench for 4" compression coupling end nuts

1-Custom wrench head attached to table

1-Plastic or Teflon ${ }^{\circledR}$ spatula or spoon

1-SCUBA cylinder

1-SCUBA regulator with high pressure gauge

1-SCUBA intermediate pressure hose ( $-10 \mathrm{ft}$ length)

with governor pressure gauge set to $-40 \mathrm{psi}$

1-Air pressure control manifold that includes:

Final pressure regulator valves (several per manifold)

Pressure gauges (1 per valve)

Low pressure hose, 6' length (1 per manifold)

Other required supplies/equipment:

Sediment sample containers or bags

Pore water sample jars

Sample labels or labeling tape

Beakers

Deionized water (DI)

Wash bottles, $500 \mathrm{ml}$

Protective gloves, glasses, clothing

Pens, pencils, markers

Centrifuge and centrifugation materials

Refrigerator

Freezer 
45 Sediment Pore-Water Toxicity Test Results, Deepwater Horizon Oil Release, Gulf of Mexico, 2010 Appendix 1

$\underline{\text { Attachment B }}$

\section{SAMPLE HISTORY DATA FORM}

Sample Designation:

Study Protocol:

Initials:

Date of acquisition:

Sample type:

How acquired (refer to sample site data sheet number, if appropriate):

$\underline{\text { Initials }} \underline{\text { Date }} \quad \underline{\text { Action Taken }}$


Date Prepared : April 10, 1990

Date Revised: July 18, 2007

\section{SEA URCHIN FERTILIZATION TOXICITY TEST}

\subsection{OBJECTIVE}

The purpose of the fertilization toxicity test with the sea urchin, Arbacia punctulata, is to determine if a sea water, pore water, sea surface microlayer, or other sample reduces fertilization of exposed gametes relative to that of gametes exposed to a reference sample. The test may also be used to determine the concentration of a test substance which reduces fertilization. Test results are reported as treatment (or concentration) which produces statistically significant reduced fertilization or as concentration of test substance which reduces fertilization by 50 percent $\left(\mathrm{EC}_{50}\right)$. This test can be performed concurrently with Sea Urchin Embryological Development Toxicity Test (CERC SOP P.648) using the same pretest and sperm and egg collection.

\subsection{TEST PREPARATION}

\subsection{Test Animals}

Gametes from the sea urchin, Arbacia punctulata are used in the sea urchin fertilization toxicity test. Animals can be collected in the field or obtained from a commercial supplier. A. punctulata can be differentiated from other species of urchins which are found in Texas by the five plates surrounding the anal opening, and by round sharp spines on the dorsal surface of the test and flattened spines surrounding the Aristotle's lantern. Urchins can be maintained easily in aquaria or other tanks with running seawater or an aquarium filter. Urchins will eat a wide variety of marine vegetation. A good diet may be provided by placing rocks from jetties (which have been colonized by diatoms and macroalgae) into the tank with the urchins. Large leaf spinach, carrots and/or romaine lettuce may be provided as a substitute. An artificial dried diet is being investigated at this time as a complete nutritional substitute for fresh foods. Cultures are maintained at $16 \pm 1{ }^{\circ} \mathrm{C}$ when gametes are not required. Temperature is gradually increased to $19 \pm 1^{\circ} \mathrm{C}$ at least one week prior to gamete collection and subsequently decreased if no further tests are planned. Photoperiod is maintained at 16 hours of light per day. Water quality parameters should be monitored weekly and salinity maintained at $30 \pm 3 \%$. Males and females should be kept in separate tanks or separated by a barrier in the same tank. 


\subsection{Dilution Water}

Milli- $\mathrm{Q}^{\circledR}$ purified water or concentrated seawater brine is used to adjust samples to $30 \%$ as described in Water Quality Adjustment of Samples (CERC SOP P.651). Concentrated seawater brine (90-110\%) is made in large batches by heating seawater to $40{ }^{\circ} \mathrm{C}$ or less in large tanks with aeration for 3-4 weeks. Brine quality will remain constant over long periods with no refrigeration. At the time of salinity adjustment, $\mathrm{pH}$, ammonia, and dissolved oxygen are also measured. Salinity adjustment and water quality data are recorded on prepared data forms.

Filtered $(0.45 \mu \mathrm{m})$ seawater adjusted to $30 \%$ is used to wash eggs and is also used for sperm and egg dilutions. The acronym MFS (for Millipore 年 filtered seawater) is used for this filtered and salinity adjusted seawater.

\subsection{Test System: Equipment}

When testing samples for potential toxicity, five replicates per treatment are recommended. One replicate is a $5 \mathrm{~mL}$ volume of sample in a disposable glass scintillation vial. When conducting a dilution series test, fifty percent serial dilutions may be made in the test vials, using MFS as the diluent.

\subsubsection{Equipment}

A list of equipment necessary for conducting this test is given in Attachment 1 (Equipment List for Fertilization Toxicity Test).

\subsubsection{Solutions}

10 percent Buffered Formalin:

$1,620 \mathrm{~mL}$ sea water

$620 \mathrm{~mL}$ formaldehyde

$6.48 \mathrm{~g} \mathrm{NaH}_{2} \mathrm{PO}_{4}$ or $\mathrm{KH}_{2} \mathrm{PO}_{4}$ (mono)

$10.5 \mathrm{~g} \mathrm{Na}_{2} \mathrm{HPO}_{4}$ or $\mathrm{K}_{2} \mathrm{HPO}_{4}$ (dibasic)

$1 \mathrm{~mL}$ needed for each replicate. Fill the dispenser.

\subsection{Collection and Preparation of Gametes}

Quality gametes must first be collected, and then diluted to the appropriate concentration for addition to the test vials. 


\subsubsection{Selection of Urchins to be Used in Toxicity Test.}

1. Take two or three females and place in shallow bowl, barely covering tests with seawater.

2. Stimulate release of eggs from gonopores of a female by touching test with electrodes from a $12 \mathrm{~V}, 0.15$ ampere transformer.

3. Collect a few eggs from between spines using a $10 \mathrm{~mL}$ disposable syringe with a large gauge blunt-tipped needle attached. Discard the first small quantity of eggs expelled from each gonopore and continue collecting. Place a 2 to 5 drops of eggs onto a scintillation vial containing $10 \mathrm{ml}$ of filtered seawater. Rinse syringe and repeat for each female.

4. Select females which have round, well developed eggs, and which do not release clumps of eggs or undeveloped ovarian tissue.

5. Place 2-4 males in shallow bowl(s) with a small amount of seawater, leaving the upper $1 / 2$ to $1 / 3$ of the animals uncovered.

6. Stimulate release of sperm from gonopores by touching test with electrodes from the $12 \mathrm{~V}, 0.15$ ampere transformer (about 30 seconds each time). If sperm is watery, reject the animal and choose another. Sperm should be the consistency of condensed milk. Collect sperm using a pastuere pipette with a rubber bulb attached.

Generally, a gamete check is performed in order to ensure that both the male and the female urchins used in the test have gametes with a high degree of viability. If the gamete check is performed, two to five females (depending on confidence in the proportion of urchins in the holding facility in good reproductive status) and at least two males should be selected using the above procedures. The check is performed by adding 5 to 7 drops of a concentrated dilution of sperm to the eggs in the scintillation vials (collected as described above) and observing the eggs under the microscope after 10 minutes. The concentrated dilution of sperm is usually made by diluting $20-50 \mu 1$ of sperm in $10 \mathrm{ml}$ of filtered seawater. If the proportion of eggs fertilized is high (95-100 percent), that female and male may be used in the pretest and test. Sperm from a number of males or females may be combined in the beginning if the gamete check reveals a number of high quality animals or the confidence is high in the quality of the gametes. Once a good male and female are selected a pretest can be conducted to determine the correct dilution of sperm to use in the test (Attachment 2). 


\subsubsection{Obtain Eggs}

1. Place selected female in large Carolina dish and add enough water to cover the urchin's test with approximately $1 \mathrm{~cm}$ of seawater. Stimulate release of eggs from female with $12 \mathrm{~V}, 0.15$ ampere transformer.

2. Collect eggs as above using the $10 \mathrm{~mL}$ syringe. Remove needle before dispensing eggs into a disposable shell vial or other clean container capable of holding 25-50 mL. Collect enough eggs for pretest and test. If female stops giving eggs readily or starts giving chunky material, cease stimulation and collection of eggs from that female.

3. Add MFS to fill shell vials, gently mixing eggs. Allow eggs to settle to bottom of vial. Remove water with a pipette. Replace water, again gently mixing the eggs.

4. Repeat washing procedure.

\subsubsection{Prepare Appropriate Egg Concentration}

1. Put approximately $100 \mathrm{~mL}$ of $30 \%$ MFS in a $250 \mathrm{~mL}$ beaker, and add enough washed eggs to bring the egg density to approximately 10,000 per $\mathrm{mL}$ If more than 400 total replicates (27 treatments) are to be tested, a larger amount of water and a correspondingly larger amount of eggs should be used. Two hundred $\mu \mathrm{L}$ of this egg solution will be used per replicate, and it is easier to maintain proper mixing and uniform egg density if there is an excess of at least 50 percent.

2. Check egg density and adjust to within approximately 9000 to 11,000 eggs per $\mathrm{mL}$, as follows. Gently swirl egg solution until evenly mixed. Using a pipette, add $1 \mathrm{~mL}$ of the solution to a vial containing nine $\mathrm{mL}$ seawater. Mix and transfer $1 \mathrm{~mL}$ of this diluted solution to a second vial containing $4 \mathrm{~mL}$ of seawater. Again, mix and transfer $1 \mathrm{~mL}$ of this diluted solution to a counting slide such as a Sedgewick-Rafter slide.

3. Using a microscope (either a compound microscope with a 10x objective or a dissecting scope may be used here), count the number of eggs on the slide. If the number is not between 180 and 220, then adjust by adding eggs or water. If egg count is $>220$ use the following formula to calculate the amount of water to add:

("egg count" - 200/200) x Current Volume of Eggs = Volume seawater to add to stock $(\mathrm{mLs})$ 
If egg count $<200$ add a small amount of eggs. Since it is less arbitrary and more likely to arrive at an acceptable count when using the water addition formula, it is better to originally overestimate the amount of eggs to add to the $100 \mathrm{~mL}$ of water.

4. Repeat steps 2 and 3 until an acceptable egg count (between 180 and 220) is obtained.

\subsubsection{Obtain Sperm}

Place selected male urchin in a large Carolina dish containing 1-2 cm of water. About half of test should be above water level. Stimulate male with $12 \mathrm{~V}$ transformer, and collect about $0.5 \mathrm{~mL}$ of unwetted sperm from between spines using a Pasteur pipette. Place sperm into a plastic microcentrifuge tube. Keep on ice until used. Wrap the microcentrifuge tube in a small piece of paper towel before storing on ice to prevent the sperm from freezing when coming in contact with the ice. Be careful not to add any water or sperm which has contacted water to the vials. High quality sperm collected dry and kept on ice will last at least eight hours without measurable decline in viability.

\subsubsection{Prepare Appropriate Sperm Dilution}

It is desirable for control fertilization to be within 60-90 percent. Although controls outside these bounds do not automatically disqualify a test, particularly if a valuable dose response is generated, the sensitivity of the test is reduced by fertilization rates greater than 90 percent and good dose responses may be difficult to obtain with less than 60 percent fertilization in controls. Density of sperm in the sperm solution should be determined with this goal in mind. Condition of the animals and length of acclimation to the aquarium may effect the chosen sperm density. The pretest (Attachment 2) may be used to calculate an appropriate sperm dilution. Generally, a dilution of between 1:10,000 and 1:2500 will result in desirable fertilization rates, if the animals are in good condition.

For example, if a sperm dilution of 1:5000 is required (as determined from the pretest), add $20 \mu \mathrm{L}$ sperm to $10 \mathrm{~mL}$ MFS. Mix thoroughly, then add $1 \mathrm{~mL}$ of this solution to $9 \mathrm{~mL}$ MFS. Sperm should not be wetted until just before starting the test. Sperm wetted more than 30 minutes before the test has begun, including sperm dilutions used in any pretest, should be discarded and a new dilution made from sperm kept on ice. 


\subsection{TEST PROCEDURES}

1. Add $50 \mu \mathrm{L}$ appropriately diluted sperm to each vial. Record the time of sperm addition. Sperm should be used within 30 minutes of wetting.

2. Incubate all test vials at $20 \pm 2^{\circ} \mathrm{C}$ for 30 minutes. At this point it is useful to set a timer for five to ten minutes prior to the end of the incubation period. This will notify the worker early enough to be ready to start the next step exactly on time.

3. While gently swirling the egg solution to maintain even mixing of eggs, use a $200 \mu \mathrm{L}$ pipetter to add $200 \mu \mathrm{L}$ diluted egg suspension to each vial. Pipette tips are cut back using a clean razor blade to prevent crushing the eggs during pipetting. Record time of egg addition.

4. Incubate for 30 minutes at $20 \pm 2^{\circ} \mathrm{C}$. The timer may be used again at this point.

5. Using the dispenser, add $0.75 \mathrm{~mL}$ of 10 percent buffered formalin to each sample.

6. Vials may now be capped and stored overnight or for several days until evaluated. Fertilization membranes are easiest to see while eggs are fairly fresh, so evaluation within two to three days may decrease the time required for evaluation.

7. If it is not possible to make the evaluations within several days or the membranes are difficult to discern, an optional technique may be employed. Make up a $200 \% \mathrm{NaCl}$ solution (pickling salt) and add 2 to 4 drops of the solution to a $1 \mathrm{~mL}$ egg sample on a microscope slide. This solution causes the egg, but not the membrane, to shrink briefly thereby making the membrane easier to see. The effect only lasts for a short time ( 5 min.) so the observations must be made immediately after the $\mathrm{NaCl}$ solution is added. If this optional technique is employed, it must be used on all samples in that test series.

\subsection{DATA COLLECTION AND TABULATION}

1. Transfer approximately $1 \mathrm{~mL}$ eggs and water from bottom of test vials to counting slide. Observe eggs using compound microscope under 100X magnification. Dark field viewing is useful here in identifying fertilization membranes.

2. Count 100 eggs/sample using hand counter with multiple keys (such as a blood cell counter), using one key to indicate fertilized eggs and another to indicate unfertilized eggs. Fertilization is defined by the presence of fertilization membrane surrounding egg.

3. Calculate fertilization percentage for each replicate test:

$$
\frac{\text { Total No. Eggs - No. Eggs Unfertilized }}{\text { Total No. Eggs }} \times 100=\text { Percent Eggs Fertilized }
$$




\subsection{DATA ANALYSIS}

Data are recorded on standardized data sheets (See Attachments 3-7). Normally, percent fertilization in each treatment is compared to an appropriate reference treatment (seawater, pore water or sea surface microlayer from an uncontaminated environment). Statistical comparisons are made using analysis of variance (ANOVA) and Dunnett's $t$-test (Sokal and Rohlf, 1981) on the arc sine square root transformed data. For multiple comparisons among treatments, Ryan's Q test (Day and Quinn, 1989) with the arc sine square root transformed data is recommended. The trimmed Spearman-Karber method with Abbott's correction is recommended to calculate $\mathrm{EC}_{50}$ values for dilution series tests (Hamilton et al., 1977)

\subsection{QUALITY CONTROL}

Quality control tests may be run using both positive and negative controls with multiple replicates (as many as desired). Typically, a reference toxicant dilution series (sodium dodecyl sulfate) is tested with each test to evaluate the effectiveness of the sperm dilution chosen. Negative controls may include a reference porewater, filtered seawater, and/or a reconstituted brine.

\subsection{TRAINING}

A trainee will conduct the test with supervision initially. Determining egg concentrations and fertilization counts are test specific activities. These functions can be performed independently after a trainee has demonstrated he or she can accurately reproduce the test.

\subsection{SAFETY}

The sea urchin fertilization toxicity test poses little risk to those performing it. Care should be taken when making and dispensing the 10 percent buffered formalin solution; use a hood if available, but make sure the test area is well ventilated. Protective gloves can be worn when pipetting or dispensing formalin or potentially toxic samples.

Care should be taken when collecting or otherwise handling sea urchins. Urchin spines are sharp and fragile and may puncture the skin and break off if handled roughly. First aid similar to treatment of wood splinters is effective in this case (removal of spine and treatment with antiseptic). Collection of sea urchins by snorkeling should not be done alone. 


\subsection{ATTACHMENTS}

Attachment 1. Equipment List for Fertilization Toxicity Test

Attachment 2. Pretest to Insure Selection of Quality Gametes

Attachment 3. Water Quality Adjustment Data Form

Attachment 4. Sea Urchin Pretest Data Sheet

Attachment 5. Sea Urchin Pretest Continuation Data Sheet

Attachment 6. Sea Urchin Fertilization/Embryological Development Toxicity Test Gamete Data Sheet

Attachment 7. Sea Urchin Fertilization Toxicity Test Fertilization Data Sheet

\subsection{REFERENCES}

Day, R.W. and G.P. Quinn. 1989. Comparisons of treatments after an analysis of variance in ecology. Ecol. Monogr. 59:433-463.

Hamilton, M.A., R.C. Russo, and R.V. Thurston. 1977. Trimmed Spearman-Karber method for estimating median lethal concentrations in toxicity bioassays. Environ. Sci. Technol. 11(7):714-719; Correction 12(4):417 (1978)

Sokal, R.R., and F.J. Rohlf. 1981. Biometry. $2^{\text {nd }}$ edition. W.H. Freeman and Company, San Francisco, CA 859 pp. 
Revised by:

Approved by:
James M. Biedenbach
Biologist

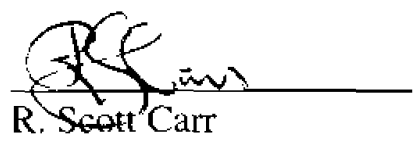

Field Station Leader

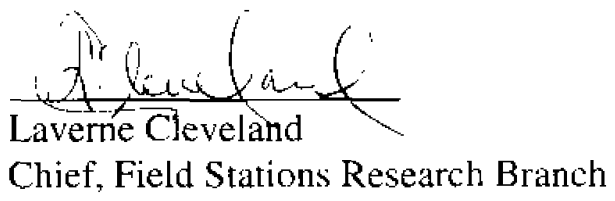

Toud Roune

Paul Heine

Quality Assurance Officer 


\section{Attachment 1}

\section{EQUIPMENT LIST FOR FERTILIZATION TOXICITY TEST}

Large Carolina dishes (at least 2)

$20 \mathrm{~mL}$ Wheaton scintillation vials (These should be type shipped with caps with polyseal cone

liners. If other brand or type is used, the vials should be tested for toxicity prior to use.) $400 \mathrm{~mL}$ beaker or wide-mouthed thermos for holding vials of sperm

$250 \mathrm{~mL}$ beakers (4)

Pasteur pipettes and latex bulbs

plastic microcentrifuge tubes

$25 \mathrm{~mL}$ shell vials or equivalent

Test tube rack (to hold shell vials)

$12 \mathrm{~V}$ transformer with pencil type electrodes

Styrofoam (or something to hold electrode tips)

$10 \mathrm{cc}$ syringe with large diameter blunt ended needle (make by grinding sharp point off the needle with a grinding stone)

Marking pens

Ice

$10-100 \mu \mathrm{L}$ pipetter

50-200 $\mu \mathrm{L}$ pipetter

$5 \mathrm{~mL}$ pipetters (2)

Counting slide such as Sedgewick-Rafter chamber

Compound microscope with 10x objective and dark field capability

Hand tally counter

Calculator

Timer for exposure / incubation periods

Buffered formalin and dispenser

Filtered $(0.45 \mu \mathrm{m})$ seawater, adjusted to $30 \%$

Data sheets

Milli-Q ${ }^{\circledR}$ reagent grade water

Approximately $100 \%$ o concentrated brine 


\section{Attachment 2}

\section{PRETEST TO INSURE SELECTION OF QUALITY GAMETES}

1. Using the procedure in section 2.4.1, select 2 to 5 females and at least 2 male urchins to be used in the pretest.

2. Fill pretest vials with five $\mathrm{mL}$ of reference water. There should be at least two vials for each combination of male, female, and pretest sperm concentration (step 4 below). For example, in a pretest with two females, one male, and six pretest sperm concentrations, 24 vials ( 2 X 2 X 6) would be needed. Arrange and mark vials accordingly in a rack.

3. Perform steps 2.4.2 (egg collection) and 2.4.3 (egg dilution) for each female urchin. Make enough volume of the egg suspension to perform the pretest and the test.

4. Perform step 2.4.4 (sperm collection) for each male urchin or male combination. Prepare a dilution series of sperm concentrations which will bracket the 60-90 percent fertilization rate in the test. Sperm dilution will depend on the health and reproductive status of the male urchin, but in most cases the following "standard dilution" should be used:

1: $250(20 \mu \mathrm{L}$ dry sperm added to $5 \mathrm{~mL}$ MFS. This concentration is used only as stock solution to make up the rest of the dilution series and is not used full strength in the pretest.)

1: 1250 ( $1 \mathrm{~mL}$ of 1:250 and $4 \mathrm{~mL}$ MFS)

1: 2500 (1 $\mathrm{mL}$ of $1: 250$ and $9 \mathrm{~mL}$ MFS)

1: 5000 (2 $\mathrm{mL}$ of $1: 2500$ and $2 \mathrm{~mL}$ MFS)

1: 7500 (2 mL of 1:2500 and $4 \mathrm{~mL}$ MFS)

$1: 10000$ (3 $\mathrm{mL}$ of $1: 7500$ and $1 \mathrm{~mL}$ MFS)

$1: 12500(1 \mathrm{~mL}$ of $1: 2500$ and $4 \mathrm{~mL}$ MFS)

Sperm must be used within 30 minutes of dilution. Leave undiluted sperm on ice and retain, because a new sperm dilution of the concentration determined in this pretest will be needed for the toxicity test. Sperm diluted for use in the pretest may not be used in the toxicity test, because the time elapsed since the addition of water is too great.

5. As in section 3.0 add $50 \mu \mathrm{L}$ of the diluted sperm to each pretest vial. Incubate for 30 minutes at approximately $20^{\circ} \mathrm{C}$, and add $200 \mu \mathrm{L}$ of the egg suspension. Incubate for another 30 minutes, then fix with $1 \mathrm{~mL}$ of the buffered formalin solution.

6. As in section 4.0, obtain a fertilization rate for the vials. There is no need to count all vials, enough vials should be counted to determine a good male/female combination, and an appropriate sperm dilution factor. If more than one male/female combination is acceptable, this is a good opportunity to choose a female which exhibits easily visible fertilization membranes or in cases where there are many samples, to combine eggs from different females . The appearance of the fertilization membranes may vary among female urchins, and presence of easily visible membranes facilitates counting. 
Attachment 3

\section{WATER QUALITY ADJUSTMENT DATA FORM}

STUDY PROTOCOL

SAMPLE DESIGNATION

\section{INITIALS}

DATE

A. Salinity Adjustment:

Initial volume (mL)

Initial salinity (\%o)

Vol. Milli-Q ${ }^{\circledR}$ water added (mL)

Vol. __ \%o brine added (mL)

percent of original sample

(initial vol./final vol. x 100)

B. Character of Sample (after salinity adjustment):

Volume (mL)

Salinity (\%o)

$\mathrm{pH}$

Dissolved oxygen (mg/L)

DO saturation ( percent)

Total ammonia (mg/L)

Sulfide (mg/L)

\section{COMMENTS}


Attachment 4

SEA URCHIN PRETEST DATA SHEET

TEST ID

STUDY PROTOCOL

\section{EGGS}

Female number:

Collection time:

Count:

\section{SPERM}

Male number:

Collection time:

Dilution start time:

\section{TEST TIMES}

Sperm in:

Eggs in:

\section{INITIALS}

DATE

SPERM DILUTION

COMMENTS

PERCENT FERTILIZATION Reference sample designation:

Female \#

Male \#

\begin{tabular}{lllll} 
Sperm Dilution & $\underline{\underline{R E P} 1}$ & $\underline{\text { REP 2 }}$ & $\underline{\underline{\text { REP 3 }}}$ & $\underline{\text { REP 4 }}$ \\
\hline & - & - & - & - \\
\hline & - & - & - & - \\
\hline & - & - & - & -
\end{tabular}

PERCENT FERTILIZATION Reference sample designation:

Female \#

$\underline{\text { Sperm dilution }}$
Male \#

$\begin{array}{llll}\underline{\underline{\text { REP 1 }}} & \underline{\underline{\text { REP 2 }}} & \underline{\underline{\text { REP 3 }}} & \underline{\text { REP 4 }} \\ - & - & - \\ - & - & - & - \\ & - & - & -\end{array}$


59 Sediment Pore-Water Toxicity Test Results, Deepwater Horizon Oil Release, 2010

Attachment 5

SEA URCHIN PRETEST CONTINUATION DATA SHEET

TEST ID

STUDY PROTOCOL
INITIALS

DATE

PERCENT FERTILIZATION Reference sample designation:

Female \# Male \#

$\underline{\text { Sperm dilution }} \underline{\underline{\text { REP 1 }}} \underline{\underline{\text { REP 2 }}} \underline{\underline{\text { REP 3 }}}$
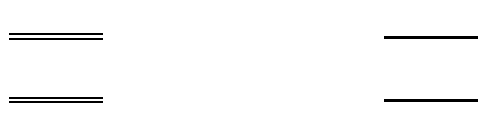

$\overline{\overline{ }} \overline{\underline{ }}$

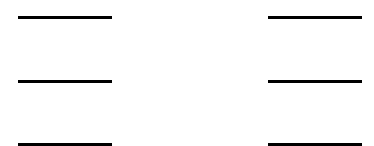

PERCENT FERTILIZATION Reference sample designation:

Female \#

Male \#

$\underline{\text { Sperm dilution }} \underline{\underline{\text { REP 1 }}} \underline{\underline{\text { REP 2 }}}$

$\overline{\underline{\underline{F}}}$

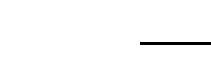

$\overline{\underline{ }}$
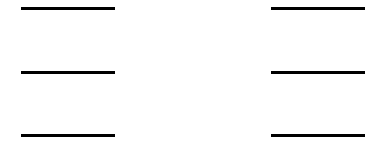

PERCENT FERTILIZATION Reference sample designation:

Female \#

Male \#

$\underline{\text { Sperm dilution }} \underline{\underline{\text { REP 1 }}} \underline{\underline{\text { REP 2 }}} \underline{\underline{\text { REP 3 }}}$
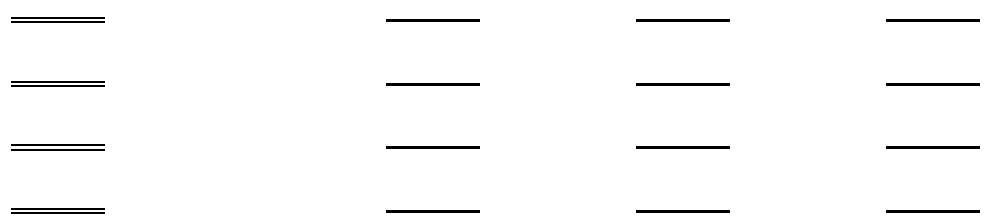

PERCENT FERTILIZATION Reference sample designation:

Female \#

Male \#

Sperm dilution

$\underline{\underline{R E P} 1}$

$\underline{\underline{R E P} 2}$

REP 3

$\underline{\underline{R E P} 4}$ 
60 Sediment Pore-Water Toxicity Test Results, Deepwater Horizon Oil Release, 2010

Attachment 6

SEA URCHIN FERTILIZATION/EMBRYOLOGICAL DEVELOPMENT TOXICITY TEST GAMETE DATA SHEET

TEST ID

STUDY PROTOCOL
INITIALS

DATE

\section{EGGS}

Collection time:

Initial count/volume:

Final count:

\section{SPERM}

Collection time:

Dilution start time:

Sperm dilution:

Test start temperature:

\section{TEST TIMES}

$\underline{\text { Box \# }} \quad \underline{\text { Sperm in: }} \quad \underline{\text { Eggs in: }} \quad \underline{\text { Formalin in: }}$


61 Sediment Pore-Water Toxicity Test Results, Deepwater Horizon Oil Release, 2010

Attachment 7

\section{SEA URCHIN FERTILIZATION TOXICITY TEST FERTILIZATION DATA SHEET}

TEST ID

STUDY PROTOCOL
INITIALS

DATE

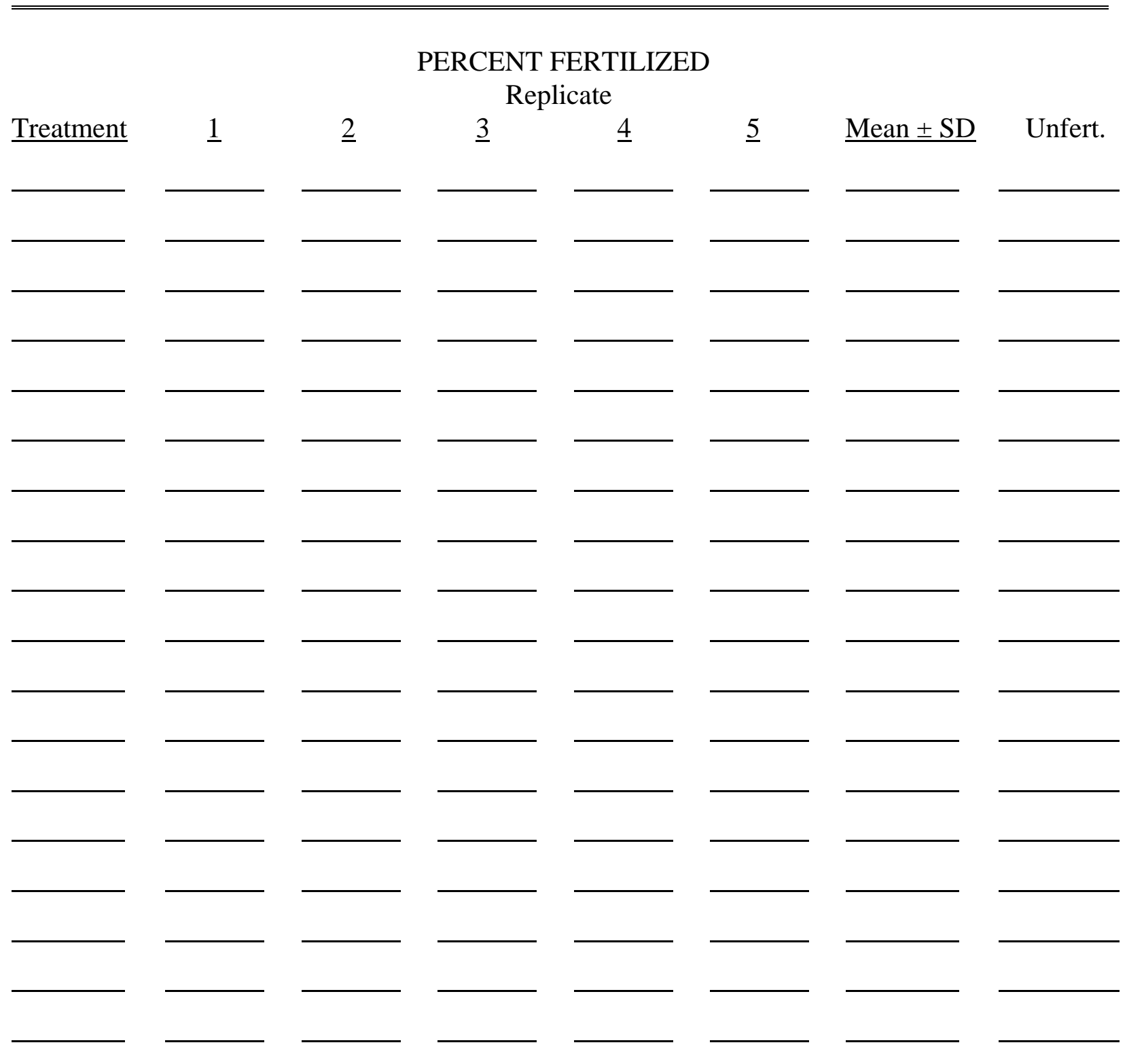


Date Prepared : April 10, 1990

Date Revised: July 18, 2007

\section{SEA URCHIN EMBRYOLOGICAL DEVELOPMENT TOXICITY TEST}

\subsection{OBJECTIVE}

The purpose of the embryological development toxicity test with the sea urchin, Arbacia punctulata, is to determine if a sea water, pore water, sea surface microlayer, or other sample affects development of exposed embryos (development arrested at an early stage or a developmental abnormality) relative to that of embryos exposed to a reference sample. The test may also be used to determine the concentration of a test substance which affects development. Test results are reported as treatment (or concentration) which produces statistically significant developmental effect. This test can be performed concurrently with Sea Urchin Fertilization Toxicity Test (CERC SOP P.647) using the same pretest and sperm and egg collection.

\subsection{TEST PREPARATION}

\subsection{Test Animals}

Gametes from the sea urchin, Arbacia punctulata are used in the sea urchin embryological development toxicity test. Animals can be collected in the field or obtained from a commercial supplier. A. punctulata can be differentiated from other species of urchins which are found in Texas by the five plates surrounding the anal opening, and by round sharp spines on the dorsal surface of the test and flattened spines surrounding the Aristotle's lantern. Urchins can be maintained easily in aquaria or other tanks with running seawater or an aquarium filter. Urchins will eat a wide variety of marine vegetation. A good diet may be provided by placing rocks from jetties (which have been colonized by diatoms and macroalgae) into the tank with the urchins. Large leaf spinach, carrots and/or romaine lettuce may be provided as a substitute. An artificial dried diet is being investigated at this time as a complete nutritional substitute for fresh foods. Cultures are maintained at $16 \pm 1^{\circ} \mathrm{C}$ when gametes are not required. Temperature is gradually increased to $19 \pm 1^{\circ} \mathrm{C}$ at least one week prior to gamete collection and subsequently decreased if no further tests are planned. Photoperiod is maintained at 16 hours of light per day. Water quality parameters should be monitored weekly and salinity maintained at $30 \pm 3 \%$. Males and females should be kept in separate tanks. 


\subsection{Dilution Water}

Milli- $\mathrm{Q}^{\circledR}$ purified water or concentrated seawater brine is used to adjust samples to $30 \%$ as described in Water Quality Adjustment of Samples (CERC SOP P.651). Concentrated seawater brine (90-110\%) is made in large batches by heating seawater to $40^{\circ} \mathrm{C}$ or less in large tanks with aeration for 3-4 weeks. Brine quality will remain constant over long periods with no refrigeration. At the time of salinity adjustment, $\mathrm{pH}$, ammonia, and dissolved oxygen are also measured. Salinity adjustment and water quality data are recorded on prepared data forms.

Filtered $(0.45 \mu \mathrm{m})$ seawater adjusted to $30 \%$ is used to wash eggs and is also used for sperm and egg dilutions. The acronym MFS (for Millipore ${ }^{\circledR}$ filtered seawater) is used for this filtered and salinity adjusted seawater.

\subsection{Test System: Equipment}

When testing samples for potential toxicity, five replicates per treatment are recommended. One replicate is a $5 \mathrm{~mL}$ volume of sample in a disposable glass scintillation vial. When conducting a dilution series test, fifty percent serial dilutions may be made in the test vials, using MFS as the diluent.

\subsubsection{Equipment}

A list of equipment necessary for conducting this test is given in Attachment 1 (Equipment List for Fertilization Toxicity Test).

\subsubsection{Solutions}

10 percent Buffered Formalin:

$1,620 \mathrm{~mL}$ sea water

$620 \mathrm{~mL}$ formaldehyde

$6.48 \mathrm{~g} \mathrm{NaH}_{2} \mathrm{PO}_{4}$ or $\mathrm{KH}_{2} \mathrm{PO}_{4}$ (mono)

$10.5 \mathrm{~g} \mathrm{Na}_{2} \mathrm{HPO}_{4}$ or $\mathrm{K}_{2} \mathrm{HPO}_{4}$ (dibasic)

$1 \mathrm{~mL}$ needed for each replicate. Fill the dispenser.

\subsection{Collection and Preparation of Gametes}

Quality gametes must first be collected, and then diluted to the appropriate concentration for addition to the test vials. 


\subsubsection{Selection of Urchins to be Used in Toxicity Test.}

1. Take two or three females and place in shallow bowl, barely covering tests with seawater.

2. Stimulate release of eggs from gonopores of a female by touching test with electrodes from a $12 \mathrm{~V}, 0.15$ ampere transformer.

3. Collect a few eggs from between spines using a $10 \mathrm{~mL}$ disposable syringe with a large gauge blunt-tipped needle attached. Discard the first small quantity of eggs expelled from each gonopore and continue collecting. Place 2 to 5 drops of eggs onto a scintillation vial containing $10 \mathrm{ml}$ of filtered seawater. Rinse syringe and repeat for each female.

4. Select females which have round, well developed eggs, and which do not release clumps of eggs or undeveloped ovarian tissue.

5. Place 2-4 males in shallow bowl(s) with a small amount of seawater, leaving the upper $1 / 2$ to $1 / 3$ of the animals uncovered.

6. Stimulate release of sperm from gonopores by touching test with electrodes from the $12 \mathrm{~V}, 0.15$ ampere transformer (about 30 seconds each time). If sperm is watery, reject the animal and choose another. Sperm should be the consistency of condensed milk. Collect sperm using a pastuere pipette with a rubber bulb attached.

Generally, a gamete check is performed in order to ensure that both the male and the female urchins used in the test have gametes with a high degree of viability. If the gamete check is performed, two to five females (depending on confidence in the proportion of urchins in the holding facility in good reproductive status) and at least two males should be selected using the above procedures. The check is performed by adding 5 to 7 drops of a concentrated dilution of sperm to the eggs in the scintillation vials (collected as described above) and observing the eggs under the microscope after 10 minutes. The concentrated dilution of sperm is usually made by diluting $20-50 \mu \mathrm{l}$ of sperm in $10 \mathrm{ml}$ of filtered seawater. If the proportion of eggs fertilized is high (95-100 percent), that female and male may be used in the pretest and test. Sperm from a number of males or females may be combined in the beginning if the gamete check reveals a number of high quality animals or the confidence is high in the quality of the gametes. Once a good male and female are selected a pretest can be conducted to determine the correct dilution of sperm to use in the test (Attachment 2). 


\subsubsection{Obtain Eggs}

1. Place selected female in large Carolina dish and add enough water to cover the urchin's test with approximately $1 \mathrm{~cm}$ of seawater. Stimulate release of eggs from female with $12 \mathrm{~V}, 0.15$ ampere transformer.

2. Collect eggs as above using the $10 \mathrm{~mL}$ syringe. Remove needle before dispensing eggs into a disposable shell vial or other clean container capable of holding 25-50 mL. Collect enough eggs for pretest and test. If female stops giving eggs readily or starts giving chunky material, cease stimulation and collection of eggs from that female.

3. Add MFS to fill shell vials, gently mixing eggs. Allow eggs to settle to bottom of vial. Remove water with a pipette. Replace water, again gently mixing the eggs.

4. Repeat washing procedure.

\subsubsection{Prepare Appropriate Egg Concentration}

1. Put approximately $100 \mathrm{~mL}$ of $30 \%$ MFS in a $250 \mathrm{~mL}$ beaker, and add enough washed eggs to bring the egg density to approximately 10,000 per $\mathrm{mL}$ If more than 400 total replicates (27 treatments) are to be tested, a larger amount of water and a correspondingly larger amount of eggs should be used. Two hundred $\mu \mathrm{L}$ of this egg solution will be used per replicate, and it is easier to maintain proper mixing and uniform egg density if there is an excess of at least 50 percent.

2. Check egg density and adjust to within approximately 9000 to 11,000 eggs per $\mathrm{mL}$, as follows. Gently swirl egg solution until evenly mixed. Using a pipette, add $1 \mathrm{~mL}$ of the solution to a vial containing nine $\mathrm{mL}$ seawater. Mix and transfer $1 \mathrm{~mL}$ of this diluted solution to a second vial containing $4 \mathrm{~mL}$ of seawater. Again, mix and transfer $1 \mathrm{~mL}$ of this diluted solution to a counting slide such as a Sedgewick-Rafter slide.

3. Using a microscope (either a compound microscope with a 10x objective or a dissecting scope may be used here), count the number of eggs on the slide. If the number is not between 180 and 220, then adjust by adding eggs or water. If egg count is > 220 use the following formula to calculate the amount of water to add:

("egg count" - 200/200) x Current Volume of Eggs = Volume seawater to add to stock (mLs) 
If egg count $<200$ add a small amount of eggs. Since it is less arbitrary and more likely to arrive at an acceptable count when using the water addition formula, it is better to originally overestimate the amount of eggs to add to the $100 \mathrm{~mL}$ of water.

4. Repeat steps 2 and 3 until an acceptable egg count (between 180 and 220) is obtained.

\subsubsection{Obtain Sperm}

Place selected male urchin in a large Carolina dish containing 1-2 cm of water. About half of test should be above water level. Stimulate male with $12 \mathrm{~V}, 0.15$ ampere transformer, and collect about $0.5 \mathrm{~mL}$ of unwetted sperm from between spines using a Pasteur pipette. Place sperm into a plastic microcentrifuge tube. Keep on ice until used. Wrap the microcentrifuge tube in a small piece of paper towel before storing on ice to prevent the sperm from freezing when coming in contact with the ice. Be careful not to add any water or sperm which has contacted water to the vials. High quality sperm collected dry and kept on ice will last at least eight hours without measurable decline in viability.

\subsubsection{Prepare Appropriate Sperm Dilution}

As in the Sea Urchin Fertilization Test, it is desirable for control fertilization to be 70-90 percent. Although controls outside these bounds do not automatically disqualify a test, particularly if a valuable dose response is generated, the chance of inducing polyspermy is increased with increased concentrations of sperm, and good dose responses may be difficult to obtain with less than 70 percent normal pluteus in controls. Density of sperm in the sperm solution should be determined with this goal in mind. Condition of the animals and length of acclimation to the aquarium may effect the chosen sperm density. The pretest (Attachment 2) may be used to calculate an appropriate sperm dilution. Generally, a dilution of between 1:1250 and 1:7500 will result in desirable fertilization rates, if the animals are in good condition.

For example, if a sperm dilution of 1:5000 is required (as determined from the pretest), add $20 \mu \mathrm{L}$ sperm to $10 \mathrm{~mL}$ MFS. Mix thoroughly, then add $1 \mathrm{~mL}$ of this solution to $9 \mathrm{~mL}$ MFS. Sperm should not be wetted until just before starting the test. Sperm wetted more than 30 minutes before the test has begun, including sperm dilutions used in any pretest, should be discarded and a new dilution made from sperm kept on ice. The quantity of sperm to be added to the egg dilution is calculated by dividing the total volume of eggs by five and adding $50 \mu \mathrm{L}$ of sperm dilution per that number. Sperm should be allowed to incubate with the eggs for 10 minutes to allow fertilization to take place. After 10 minutes, eggs should be evaluated under $100 \mathrm{X}$ magnification for fertilization membranes. If 
70-90 percent of the eggs are fertilized, the embryos can be pipetted into the test vials. If the percentage is lower than 70 percent, additional sperm may be added and/or more time allowed for fertilization. If the fertilization does not increase above 70 percent after 30 minutes, the embryos should be discarded and new gametes selected for use. Embryos should not be allowed to undergo division before pipetting them into the test vials.

\subsection{TEST PROCEDURES}

1. Just before the embryos are to be used, add $2 \mathrm{~mL}$ of a penicillin-G stock solution (5000 units/mL) per $100 \mathrm{~mL}$ of eggs in the fertilized embryo suspension. The addition of penicillin to the embryological development test has been shown to be beneficial in evaluation of the stages of development by inhibiting bacterial growth which can cause the embryos to disintegrate before the test is terminated. The penicillin stock solution is prepare by diluting $296 \mathrm{mg}$ of Penicillin-G sodium salt (1690 units $/ \mathrm{mg}$ ) in $100 \mathrm{~mL}$ of MFS and mixing until dissolved. The addition of $2 \mathrm{~mL} / 100 \mathrm{~mL}$ of eggs will result in a final concentration of 4 units $/ \mathrm{mL}$ in each replicate. The number of units of penicillin per $\mathrm{mg}$ of penicillin-G sodium salt is variable with each lot. Thus, the quantity added to the stock will change in order to keep the final concentration at 4 units $/ \mathrm{mL}$.

2. While gently swirling the embryo solution to maintain even mixing, use a $200 \mu \mathrm{L}$ pipetter to add $200 \mu \mathrm{L}$ diluted embryo suspension to each vial. Record time of embryo addition.

3. Incubate all test vials at $20 \pm 1^{\circ} \mathrm{C}$ for 48 hours.

4. Using the dispenser, add $0.75 \mathrm{~mL} 10$ percent buffered formalin to each vial.

5. Vials may now be capped and stored overnight or for several days until evaluated.

\subsection{DATA COLLECTION AND TABULATION}

1. Transfer approximately $1 \mathrm{~mL}$ embryos and water from bottom of test vials to counting slide. Observe embryos using a compound microscope under 100X magnification.

2. Count 100 embryos/sample using hand counter with multiple keys (such as a blood cell counter), using one key to indicate normally developed pluteus larvae and others to indicate unfertilized eggs, embryos arrested in earlier developmental stages, and other abnormalities or for more efficient data collection, stages other than pluteus and abnormalities may be lumped together and counted on one key. Attachment 3 has a list of developmental stages and drawings of each.

3. Calculate the proportion of normal plutei for each replicate test: 
Number normal plutei X $100=$ Percent normal plutei

Total no. eggs/embryos

\subsection{DATA ANALYSIS}

Data are recorded on standardized data sheets (See Attachments 4-9). Normally, percent normal development (normal plutei) in each treatment is compared to an appropriate reference treatment (seawater, pore water or sea surface microlayer from an uncontaminated environment). Statistical comparisons are made using analysis of variance (ANOVA) and Dunnett's $t$-test (Sokal and Rohlf, 1981) on the arc sine square root transformed data. For multiple comparisons among treatments, Ryan's Q test (Day and Quinn, 1989) with the arc sine square root transformed data is recommended. The trimmed Spearman-Karber method with Abbott's correction is recommended to calculate $\mathrm{EC}_{50}$ values for dilution series tests (Hamilton et al., 1977)

\subsection{QUALITY CONTROL}

Quality control tests may be run using both positive and negative controls with multiple replicates (as many as desired). Typically, a reference toxicant dilution series (sodium dodecyl sulfate) is tested with each test to evaluate the gametes chosen. Negative controls may include a reference porewater, filtered seawater, and/or a reconstituted brine.

\subsection{TRAINING}

A trainee will conduct the test with supervision initially. Determining egg concentrations, embryological stages and counts are test specific activities. These functions can be performed independently after a trainee has demonstrated he or she can accurately reproduce the test.

\subsection{SAFETY}

The sea urchin embryological development toxicity test poses little risk to those performing it. Care should be taken when making and dispensing the 10 percent buffered formalin solution; use a hood if available, but make sure the test area is well ventilated. Protective gloves can be worn when pipetting or dispensing formalin or potentially toxic samples.

Care should be taken when collecting or otherwise handling sea urchins. Urchin spines are sharp and fragile and may puncture the skin and break off if handled roughly. First aid similar to treatment of wood splinters is effective in this case (removal of spine and treatment with antiseptic). Collection of sea urchins by snorkeling should not be done alone. 


\subsection{ATTACHMENTS}

Attachment 1. Equipment List for Embryological Development Toxicity Test

Attachment 2. Pretest to Insure Selection of Quality Gametes

Attachment 3. Development of Sea Urchin Eggs to Pluteus Larvae

Attachment 4. Water Quality Adjustment Data Form

Attachment 5. Sea Urchin Pretest Data Sheet

Attachment 6. Sea Urchin Pretest Continuation Data Sheet

Attachment 7. Sea Urchin Fertilization/Embryological Development Toxicity Test Gamete Data Sheet

Attachment 8. Sea Urchin Embryological Development Test Data Sheet

\subsection{REFERENCES}

Day, R.W. and G.P. Quinn. 1989. Comparisons of treatments after an analysis of variance in ecology. Ecol. Monogr. 59:433-463.

Hamilton, M.A., R.C. Russo, and R.V. Thurston. 1977. Trimmed Spearman-Karber method for estimating median lethal concentrations in toxicity bioassays. Environ. Sci. Technol. 11(7):714-719; Correction 12(4):417 (1978)

Sokal, R.R., and F.J. Rohlf. 1981. Biometry. $2^{\text {nd }}$ edition. W.H. Freeman and Company, San Francisco, CA 859 pp. 
70 Sediment Pore-Water Toxicity Test Results, Deepwater Horizon Oil Release, Gulf of Mexico, 2010 Appendix 3

Revised by:

Approved by:

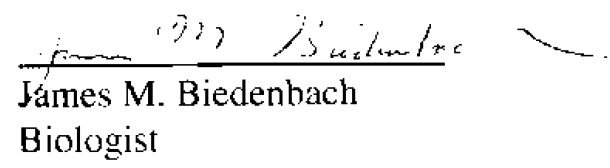

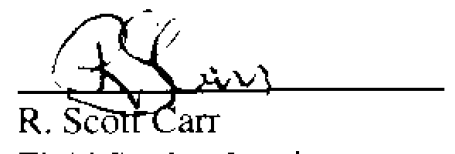

Field Station Leader
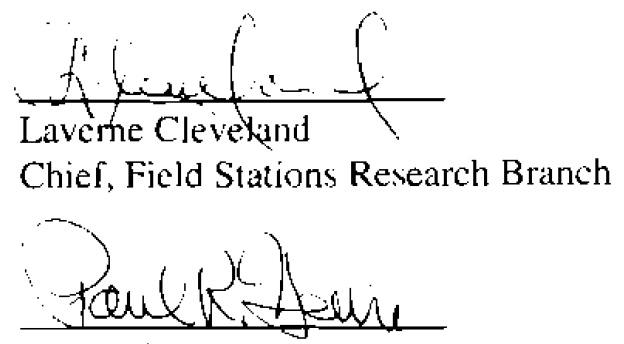

Paul Heine

Quality Assurance Otficer 


\section{Attachment 1}

\section{EQUIPMENT LIST FOR EMBRYOLOGICAL DEVELOPMENT TOXICITY TEST}

Large Carolina dishes (at least 2)

$20 \mathrm{~mL}$ Wheaton scintillation vials (These should be type shipped with caps with polycone liners. If other brand or type is used, the vials should be tested for toxicity prior to use.) $400 \mathrm{~mL}$ beaker or wide-mouthed thermos for holding vials of sperm $250 \mathrm{~mL}$ beakers (4)

Pasteur pipettes and latex bulbs plastic microcentrifuge tubes $25 \mathrm{~mL}$ shell vials or equivalent Test tube rack (to hold shell vials) $12 \mathrm{~V}$ transformer with pencil type electrodes Styrofoam (or something to hold electrode tips)

$10 \mathrm{cc}$ syringe with large diameter blunt ended needle (make by grinding sharp point off the needle with a grinding stone)

Marking pens

Ice

10-100 $\mu \mathrm{L}$ pipetter

50-200 $\mu \mathrm{L}$ pipetter

$5 \mathrm{~mL}$ pipetters (2)

Counting slide such as Sedgewick-Rafter chamber

Compound microscope with 10x objective and dark field capability

Hand tally counter

Calculator

Timer for exposure / incubation periods

Buffered formalin and dispenser

Filtered $(0.45 \mu \mathrm{m})$ seawater, adjusted to $30 \%$

Data sheets

Milli-Q ${ }^{\circledR}$ reagent grade water

Approximately $100 \%$ concentrated brine 


\section{Attachment 2}

\section{PRETEST TO INSURE SELECTION OF QUALITY GAMETES}

1. Using the procedure in section 2.4.1, select 2 to 5 females and at least 2 male urchins to be used in the pretest.

2. Fill pretest vials with five $\mathrm{mL}$ of reference water. There should be at least two vials for each combination of male, female, and pretest sperm concentration (step 4 below). For example, in a pretest with two females, one male, and six pretest sperm concentrations, 24 vials ( 2 X 2 X 6) would be needed. Arrange and mark vials accordingly in a rack.

3. Perform steps 2.4.2 (egg collection) and 2.4.3 (egg dilution) for each female urchin. Make enough volume of the egg suspension to perform the pretest and the test.

4. Perform step 2.4.4 (sperm collection) for each male urchin or male combination. Prepare a dilution series of sperm concentrations which will bracket the 60-90 percent fertilization rate in the test. Sperm dilution will depend on the health and reproductive status of the male urchin, but in most cases the following "standard dilution" should be used:

1: $250 \quad(20 \mu \mathrm{L}$ dry sperm added to $5 \mathrm{~mL}$ MFS. This concentration is used only as stock solution to make up the rest of the dilution series and is not used full strength in the pretest.)

1: 1250 ( $1 \mathrm{~mL}$ of $1: 250$ and $4 \mathrm{~mL}$ MFS)

1: 2500 (1 $\mathrm{mL}$ of $1: 250$ and $9 \mathrm{~mL}$ MFS)

1: 5000 ( $2 \mathrm{~mL}$ of $1: 2500$ and $2 \mathrm{~mL} \mathrm{MFS})$

1: 7500 ( $2 \mathrm{~mL}$ of 1:2500 and $4 \mathrm{~mL}$ MFS)

$1: 10000$ (3 $\mathrm{mL}$ of $1: 7500$ and $1 \mathrm{~mL}$ MFS)

$1: 12500$ ( $1 \mathrm{~mL}$ of $1: 2500$ and $4 \mathrm{~mL}$ MFS)

Sperm must be used within 30 minutes of dilution. Leave undiluted sperm on ice and retain, because a new sperm dilution of the concentration determined in this pretest will be needed for the toxicity test. Sperm diluted for use in the pretest may not be used in the toxicity test, because the time elapsed since the addition of water is too great.

5. As in section 3.0 add $50 \mu \mathrm{L}$ of the diluted sperm to each pretest vial. Incubate for 30 minutes at approximately $20^{\circ} \mathrm{C}$, and add $200 \mu \mathrm{L}$ of the egg suspension. Incubate for another 30 minutes, then fix with $1 \mathrm{~mL}$ of the buffered formalin solution.

6. As in section 4.0, obtain a fertilization rate for the vials. There is no need to count all vials, enough vials should be counted to determine a good male/female combination, and an appropriate sperm dilution factor. If more than one male/female combination is acceptable, this is a good opportunity to choose a female which exhibits easily visible fertilization membranes or in cases where there are many samples, to combine eggs from different females . The appearance of the fertilization membranes may vary among female urchins, and presence of easily visible membranes facilitates counting. 


\section{Attachment 3}

\section{DEVELOPMENT OF SEA URCHIN EGGS TO PLUTEUS LARVAE}

The development of sea urchin eggs from fertilization to pluteus larvae normally occurs in approximately 48 hours. Although development is a continuous process of mitosis and cellular differentiation, developmental biology defines distinct stages of development by gross morphological characteristics. For the purpose of the Sea Urchin Embryological Development Test, six stages are defined and used in the characterization of embryos (Drawings on following page).

1. Unfertilized egg - single cell which appears dense and lacks a fertilization membrane.

2. Fertilized egg - egg with a distinct fertilization membrane which appears as a thin band lying slightly away from the central egg. The early stages of cell division are included in this group.

3. Blastula - spherical, "hollow-ball" stage which is ciliated and becomes free-swimming by breaking out of the fertilization membrane.

4. Early gastrula - beginnings of invagination of the blastula wall are evident. Cells move inward (invaginate) to form a central cavity (archenteron). Early gastrula includes embryos with the earliest stages of invagination and continues until the archenteron reaches approximately two-thirds of the diameter of the embryo.

5. Late gastrula - gastrula in which archenteron has developed in length to two-thirds of the embryo diameter and has begun to differentiate and bend towards and break through the embryo wall. Included are the later stages (prism) with primitive gut (complete digestive system), early skeletal rod development, and beginnings of deltoid shape formation.

6. Pluteus - deltoid-shaped larval stage with complete digestive system, skeletal rods, and growth of projecting arms. 
74 Sediment Pore-Water Toxicity Test Results, Deepwater Horizon Oil Release, Gulf of Mexico, 2010 Appendix 3

\section{Attachment 3 Continued}
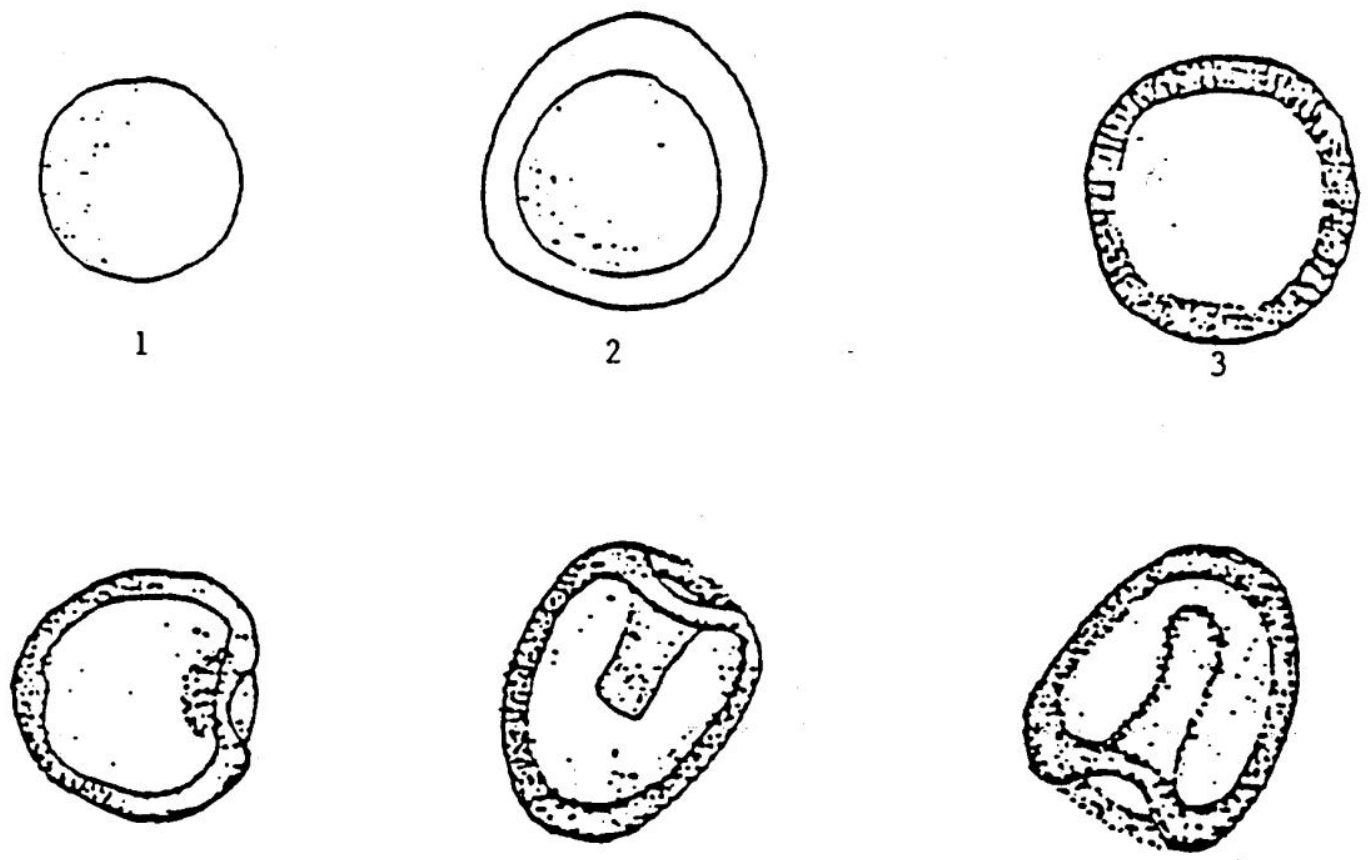

$4 b$

$5 a$
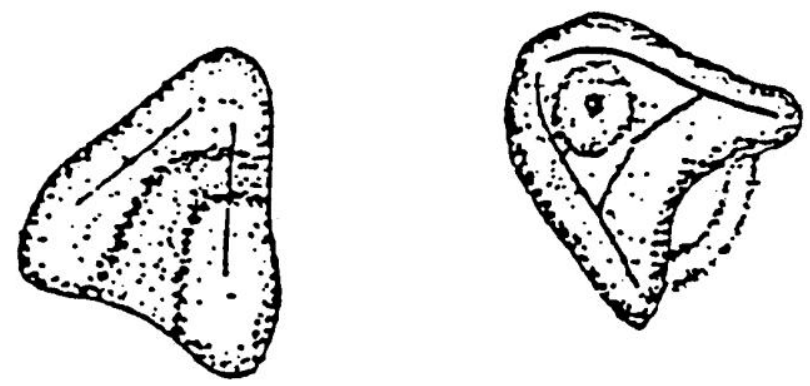

$5 b$

$6 a$

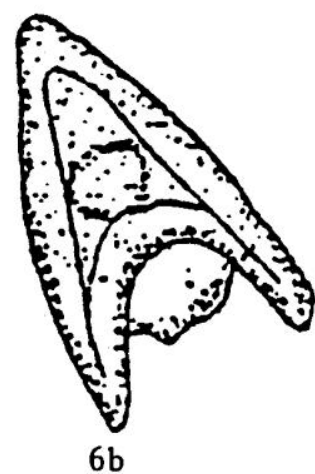

Figure 3-1. Stages in development of sea urchin, from unfertilized egg to pluteus larvae. Numbers relate to descriptions on previous page. 


\section{Attachment 4}

\section{WATER QUALITY ADJUSTMENT DATA FORM}

STUDY PROTOCOL INITIALS

SAMPLE DESIGNATION

A. Salinity Adjustment:

Initial volume $(\mathrm{mL})$

Initial salinity $(\%)$

Vol. Milli- $\mathrm{Q}^{\circledR}$ water added $(\mathrm{mL})$

Vol. __ \%o brine added (mL)

percent of original sample

(initial vol./final vol. x 100)

B. Character of Sample (after salinity adjustment):

Volume (mL)

Salinity (\%o)

$\mathrm{pH}$

Dissolved oxygen (mg/L)

DO saturation ( percent)

Total ammonia (mg/L)

Sulfide (mg/L)

\section{COMMENTS}


Attachment 5

\section{SEA URCHIN PRETEST DATA SHEET}

TEST ID

STUDY PROTOCOL

\section{EGGS}

Female number:

Collection time:

Count:

\section{SPERM}

Male number:

Collection time:

Dilution start time:

\section{TEST TIMES}

Sperm in:

Eggs in:

\section{INITIALS}

DATE

SPERM DILUTION

COMMENTS

PERCENT FERTILIZATION Reference sample designation:

Female \# Male \# $\underline{\text { Sperm Dilution }}$

$\underline{\underline{R E P} 1}$

REP 2

$\underline{\underline{R E P} 3} \quad \underline{\underline{R E P} 4}$
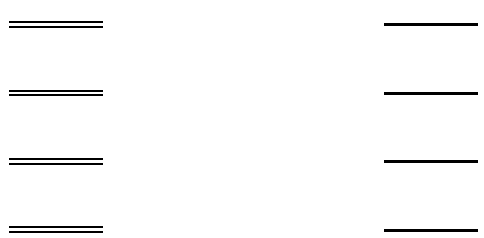

PERCENT FERTILIZATION Reference sample designation:

Female \# Male \#

\begin{tabular}{|c|c|c|c|}
\hline$\underline{\text { Sperm dilution }}$ & REP 1 & REP 2 & REP 3 \\
\hline & & & \\
\hline & & & \\
\hline & & & \\
\hline
\end{tabular}


Attachment 6

\section{SEA URCHIN PRETEST CONTINUATION DATA SHEET}

TEST ID

STUDY PROTOCOL
INITIALS

DATE

PERCENT FERTILIZATION Reference sample designation:

Female \#

Male \#

$\underline{\text { Sperm dilution }} \underline{\underline{\text { REP 1 }}} \underline{\underline{\text { REP 2 }}} \quad \underline{\underline{\text { REP 4 }}}$

PERCENT FERTILIZATION Reference sample designation:

Female \#

Male \#

$\underline{\text { Sperm dilution }} \underline{\underline{\text { REP 1 }}} \underline{\underline{\text { REP 2 }} \quad \underline{\underline{R E P ~ 3 ~}} \quad \underline{\underline{R E P ~ 4 ~}}}$

$\overline{\overline{2}}$

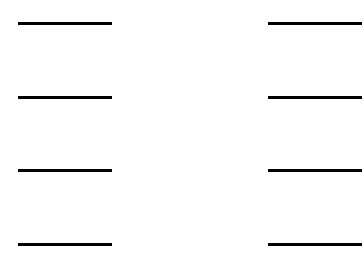

PERCENT FERTILIZATION Reference sample designation:

Female \#

Male \#

$\underline{\text { Sperm dilution }} \underline{\underline{\text { REP 1 }}} \underline{\underline{\text { REP } 2}} \quad \underline{\underline{\text { REP } 4}}$
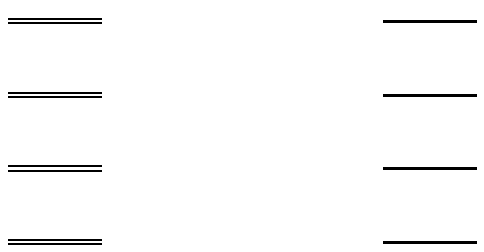

PERCENT FERTILIZATION Reference sample designation:

Female \#

Male \#

$\underline{\text { Sperm dilution }}$

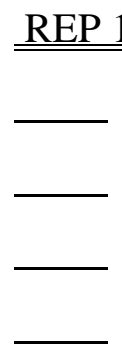

$\underline{\underline{R E P} 2}$

REP 3

REP 4 
Attachment 7

SEA URCHIN FERTILIZATION/EMBRYOLOGICAL DEVELOPMENT TOXICITY TEST GAMETE DATA SHEET

TEST ID

STUDY PROTOCOL
INITIALS

DATE

\section{EGGS}

Collection time:

Initial count/volume:

Final count:

\section{SPERM}

Collection time:

Dilution start time:

Sperm dilution:

Test start temperature:

\section{TEST TIMES}

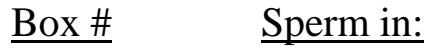

Eggs in:

Formalin in:

COMMENTS 
79 Sediment Pore-Water Toxicity Test Results, Deepwater Horizon Oil Release, Gulf of Mexico, 2010

Attachment 8

\section{SEA URCHIN EMBRYOLOGICAL DEVELOPMENT TOXICITY TEST DATA SHEET}

TEST ID

STUDY PROTOCOL

$$
\text { INITIALS }
$$

DATE

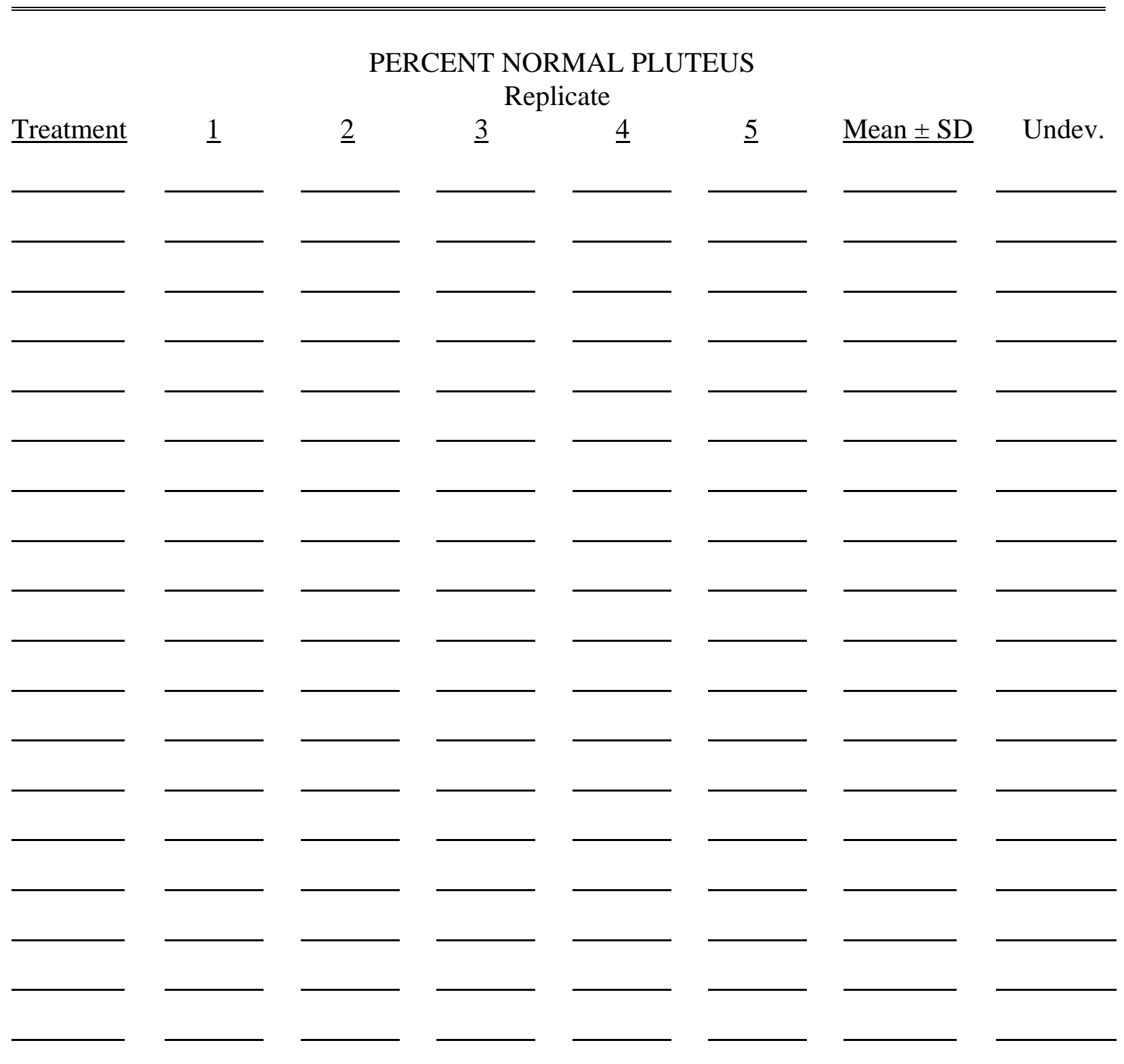


Publishing support provided by:

Rolla Publishing Service Center

For more information concerning this publication, contact: Director, USGS Columbia Environmental Research Center 4200 New Haven Road

Columbia, M0 65201

(573) 875-5399

Or visit the Columbia Environmental Research Center Web site at: http://www.cerc.usgs.gov 
